TRANSACTIONS OF THE

AMERICAN MATHEMATICAL SOCIETY

Volume 363, Number 8, August 2011, Pages 4225-4262

S 0002-9947(2011)05209-8

Article electronically published on March 15, 2011

\title{
HAUSDORFF MEASURES AND FUNCTIONS OF BOUNDED QUADRATIC VARIATION
}

\author{
D. APATSIDIS, S. A. ARGYROS, AND V. KANELLOPOULOS
}

\begin{abstract}
To each function $f$ of bounded quadratic variation we associate a Hausdorff measure $\mu_{f}$. We show that the map $f \rightarrow \mu_{f}$ is locally Lipschitz and onto the positive cone of $\mathcal{M}[0,1]$. We use the measures $\left\{\mu_{f}: f \in V_{2}\right\}$ to determine the structure of the subspaces of $V_{2}^{0}$ which either contain $c_{0}$ or the square stopping time space $S^{2}$.
\end{abstract}

\section{INTRODUCTION}

The functions of bounded quadratic variation, introduced by N. Wiener in [22], have been extensively studied in their own right as well as for their applications. For example, related results can be found in [4, [5, [9], [19] and also in the monograph [8] where several applications are included.

In the present work we study the subspace structure of $V_{2}^{0}$. In the sequel we shall denote by $V_{2}$ the space of all real-valued functions $f$ with bounded quadratic variation, defined on the unit interval and satisfying $f(0)=0 ; V_{2}$ endowed with the quadratic variation norm is a Banach space. The aforementioned space $V_{2}^{0}$ is a separable subspace of $V_{2}$ of significant importance; it is defined as the closed subspace of $V_{2}$ containing all the square absolutely continuous functions, a concept introduced by R. E. Love in the early 1950s (cf. [17).

The space $V_{2}^{0}$ was introduced by S. V. Kisliakov in [14 as an isometric version of Lindenstrauss' space $J F$. His aim was to provide easier proofs of the fundamental properties of $J F ; V_{2}^{0}$ is separable, not containing $\ell_{1}$ and with non-separable dual. These properties were the most distinctive ones for $J F$, as such a space answers in the negative a problem posed by S. Banach. Earlier R.C. James [11] had presented the James Tree $(J T)$ space which is the analogue of $V_{2}^{0}$ in the frame of the sequence spaces. It is notable that the class of the separable Banach spaces not containing $\ell_{1}$ and with non-separable dual, which appears as an exotic subclass of Banach spaces, includes spaces, such as $V_{2}^{0}$, naturally arising from other branches of Analysis.

Kisliakov also proved an important relation between the spaces $V_{2}^{0}$ and $V_{2}$. Namely, $V_{2}$ naturally coincides with the second dual of $V_{2}^{0}$ and moreover the w*topology on the bounded subsets of $V_{2}$ coincides with the topology of pointwise convergence. Among the consequences of the preceding remarkable property is that every $f \in V_{2}$ is the pointwise limit of a bounded sequence $\left(f_{n}\right)_{n}$ from $V_{2}^{0}$ (cf.

Received by the editors March 31, 2009 and, in revised form July 14, 2009.

2000 Mathematics Subject Classification. Primary 28A78, 46B20, 46B26.

Key words and phrases. Hausdorff measures, functions of bounded $p$-variation, Banach spaces with non-separable dual, James Function space.

This research was supported by PEBE 2007 . 
[18). The variety of the classical Banach spaces which are isomorphic to a subspace of $V_{2}^{0}$ is large and rather unexpected. Indeed, beyond the space $\ell_{2}$ which is the most relative to $V_{2}^{0}$, as was stated in [15] and proved later in [3] and [6], the space $c_{0}$ is also isomorphic to a subspace of $V_{2}^{0}$. Moreover for all $2 \leq p<\infty$, the space $\ell_{p}$ shares the same property (cf. [6]).

The study of the subspace structure of $V_{2}^{0}$ was initiated in [3] and [6] and continued in [1]. From our point of view a sufficient understanding of $V_{2}^{0}$ requires answers to the following problems.

Problem 1. Let $X$ be a reflexive subspace of $V_{2}^{0}$. Does there exist some $2 \leq p<\infty$ such that $\ell_{p}$ is isomorphically embedded into $X$ ?

As we have mentioned earlier, all $\ell_{p}, 2 \leq p<\infty$, are embedded into $V_{2}^{0}$. Also, in [6] it was shown that no $\ell_{p}, 1 \leq p<2$ is isomorphic to a subspace of $V_{2}^{0}$. It is worth pointing out that the proof of the embedding of $\ell_{p}, 2 \leq p<\infty$ is rather indirect and uses the quadratic time stopping space $S^{2}$, which is one of the central objects of the present paper. The space $S^{2}$ is the square convexification of the stopping time space $S^{1}$. The latter space was defined by H. P. Rosenthal as the unconditional analogue of the space $L^{1}(\lambda)$. Both spaces (i.e. $S^{1}, S^{2}$ ) belong to the wider class of the spaces $S^{p}, 1 \leq p<\infty$ introduced by S. Buechler's Ph.D. Thesis ([6]). We recall the definition. We denote by $2^{<\mathbb{N}}$ the dyadic tree and by $c_{00}\left(2^{<\mathbb{N}}\right)$ the vector space of all real-valued functions defined on $2^{<\mathbb{N}}$ with finite support. For $1 \leq p<\infty$ we define the $\|\cdot\|_{S^{p}}$ on $c_{00}\left(2^{<\mathbb{N}}\right)$ as follows. For $x \in c_{00}\left(2^{<\mathbb{N}}\right)$, we set

$$
\|x\|_{S^{p}}=\sup \left(\sum_{s \in A}|x(s)|^{p}\right)^{1 / p}
$$

where the supremum is taken over all antichains $A$ of $2^{<\mathbb{N}}$. The space $S^{p}$ is the completion of $\left(c_{00}\left(2^{<\mathbb{N}}\right),\|\cdot\|_{S^{p}}\right)$. The space $S^{1}$ has an unconditional basis and G. Schechtman, in an unpublished work, showed that it contains all $\ell_{p}, 1 \leq p<\infty$. This result was extended in [6] to all $S^{p}$ spaces by showing that for every $p \leq q, \ell_{q}$ is embedded into $S^{p}$. An excellent and detailed study of the stopping time space $S^{1}$, in fact in a more general setting, is included in N. Dew's Ph.D. Thesis (7]). The interested reader will also find there, among other things, a proof of Schechtman's unpublished result. Let us also point out that the analogous problem to Problem 1 for the spaces $S^{p}, 1 \leq p<\infty$ also remains open. An important result in [6] shows that $S^{2}$ is isomorphic to a subspace of $V_{2}^{0}$, and this actually yields that $V_{2}^{0}$ contains isomorphs of all $\ell_{p}, 2 \leq p<\infty$. Before closing our discussion for Problem 1, let us also note that for every infinite chain $C$ of $2^{<\mathbb{N}}$ the subspace of $S^{2}$ generated by $\left\{e_{s}: s \in C\right\}$ is isomorphic to $c_{0}$, while for every infinite antichain $A$ the corresponding one is isomorphic to $\ell_{2}$. Thus, if a subspace $X$ of $V_{2}^{0}$ contains an isomorph of $S^{2}$, then it contains all possible classical spaces that are embedded into $V_{2}^{0}$.

Our next two problems concern non-reflexive subspaces of $V_{2}^{0}$. Let us begin with a result from [3] which asserts that every non-reflexive subspace $X$ of $V_{2}^{0}$ contains an isomorph of $\ell_{2}$ or $c_{0}$. To see this, we start with some $f \in X^{* *} \backslash X$, where $X^{* *}$ is considered as a subspace of $V_{2}$. Since $X$ does not contain $\ell_{1}$, OdellRosenthal's theorem (cf. [18) yields that there exists a bounded sequence $\left(f_{n}\right)_{n}$ in $X$ pointwise converging to $f$. If $f$ is discontinuous, then there exists a sequence $\left(g_{k}\right)_{k}, g_{k}=f_{n_{k}}-f_{m_{k}}$ equivalent to the $\ell_{2}$ basis and hence $\ell_{2}$ is embedded into $X$. 
The case of a continuous $f$ is more interesting. As is shown in [3], such an $f$ is a difference of bounded semicontinuous functions (DBSC) when $f$ is considered as a

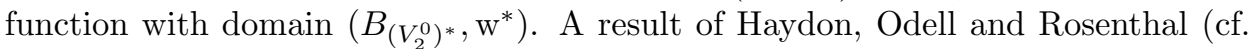
[10] ) yields that the sequence $\left(f_{n}\right)_{n}$ has a convex block subsequence $\left(g_{n}\right)_{n}$ equivalent to the summing basis of $c_{0}$. Let us note that the existence of a continuous function $f$ in $X^{* *} \backslash X$ is actually equivalent to the embedding of $c_{0}$ into $X$.

The second problem concerns subspaces of $V_{2}^{0}$ with non-separable dual and it is stated as follows.

Problem 2. Is it true that every subspace $X$ of $V_{2}^{0}$ with $X^{*}$ non-separable contains an isomorph of $V_{2}^{0}$ itself? Moreover, is every complemented subspace $X$ of $V_{2}^{0}$ with non-separable dual isomorphic to $V_{2}^{0}$ ?

An affirmative answer to the second part of Problem 2 yields that $V_{2}^{0}$ is a primary space. In [1 it has been shown that the corresponding problem in James' space $J T$ has an affirmative answer. This evidence supports the possibility for a positive solution to Problem 2. It is worth mentioning that, as is shown in [1, every subspace $X$ of $V_{2}^{0}$ with non-separable dual contains the space $T F$. This is a sequence space with non-separable dual, introduced in [1. It is isomorphic to any subspace of $V_{2}^{0}$ generated by a tree family $\left(f_{s}\right)_{s \in 2<\mathbb{N}}$ of trapezoids. The latter spaces were introduced in [6] for showing that $V_{2}^{0}$ does not contain isomorphs of $J T$. In [1] it is also stated without proof that $S^{2}$ is embedded into $T F$ which, as we have already mentioned, yields that every subspace of $V_{2}^{0}$ with non-separable dual contains isomorphs of all possible classical spaces that are embedded in $V_{2}^{0}$. In the present paper we give a proof of the embedding of $S^{2}$ into $T F$, granting from [6] that $c_{0}$ is embedded into $T F$.

The third problem concerns subspaces of $V_{2}^{0}$ with non-separable second dual.

Problem 3. Is it true that every subspace $X$ of $V_{2}^{0}$ with $X^{* *}$ non-separable contains $c_{0}$ ?

The main goal of the present work is to provide a positive answer to this problem. Before we start explaining our proof, we point out that the preceding results on subspaces $X$ with non-separable dual reduce the problem to those $X$ with $X^{*}$ separable and $X^{* *}$ non-separable. Also, as we noted above, the embedding of $c_{0}$ into $X$ is equivalent to the existence of a function $f \in\left(X^{* *} \backslash X\right) \cap C[0,1]$. In the early stages of our engagement to this problem we observed that when $X^{*}$ is separable, the set $D_{X^{* *}}=\left\{t \in[0,1]: \exists f \in X^{* *}, \operatorname{osc} f(t)>0\right\}$ is at most countable, a fact supporting an affirmative solution to the problem. However we had no further progress until the moment where we discovered a new concept which plays a key role in our approach. This is a Hausdorff type measure $\mu_{f}$ associated to every $f \in V_{2}$. The measure $\mu_{f}$ is defined as follows. First we introduce some notation.

Given $f:[0,1] \rightarrow \mathbb{R}$ and $\mathcal{P}=\left\{t_{0}<\ldots<t_{p}\right\} \subseteq[0,1]$, with $p \geq 1$, let $\|\mathcal{P}\|_{\max }=$ $\max \left\{t_{i+1}-t_{i}: 0 \leq i \leq p-1\right\}$ and $v_{2}^{2}(f, \mathcal{P})=\sum_{i=0}^{p-1}\left(f\left(t_{i+1}\right)-f\left(t_{i}\right)\right)^{2}$. For every $f \in V_{2}$ and for every interval $I$ of $[0,1]$ we set

$$
\widetilde{\mu}_{f}(I)=\inf _{\delta>0} \widetilde{\mu}_{f, \delta}(I),
$$

where for each $\delta>0, \widetilde{\mu}_{f, \delta}(I)=\sup \left\{v_{2}^{2}(f, \mathcal{P}): \mathcal{P} \subseteq I\right.$ and $\left.\|\mathcal{P}\|_{\max }<\delta\right\}$. The collection $\left\{\widetilde{\mu}_{f}(I): I\right.$ is an interval of $\left.[0,1]\right\}$ defines an outer measure and $\mu_{f}$ is the regular measure induced by $\widetilde{\mu}_{f}$ on the Borel subsets of $[0,1]$. We should mention 
that $\mathrm{N}$. Wiener himself had also considered the quantity $\sqrt{\widetilde{\mu}_{f}[0,1]}$, pointing out that it is a seminorm on $V_{2}$. The measure $\mu_{f}$ incorporates a sufficient amount of information concerning the function $f$. Thus $\mu_{f}=0$ if and only if $f \in V_{2}^{0}, \mu_{f}$ is continuous (diffuse) if and only if $f$ is continuous and also the discrete (atomic) part of $\mu_{f}$ is supported by the points of discontinuity of $f$. Furthermore the following hold.

Proposition 1. Let $f \in V_{2}$. Then the set of the points of differentiability of $f$ has $\mu_{f}$-measure zero.

As a consequence we obtain the following.

Corollary. Let $f$ be a continuous function in $V_{2}$. If the set of all non-differentiability points of $f$ is at most countable, then $f$ belongs to $V_{2}^{0}$. Moreover if $f \in\left(V_{2} \backslash\right.$ $\left.V_{2}^{0}\right) \cap C[0,1]$, then the set of all non-differentiability points of $f$ contains a perfect set.

The second result concerns the variety of the elements of $V_{2}$.

Proposition 2. Let $\Phi: V_{2} \rightarrow \mathcal{M}^{+}[0,1]$ be the function that maps $f$ to $\mu_{f}$. Then $\Phi$ is locally Lipschitz and onto. In particular for every continuous positive measure $\mu \in \mathcal{M}^{+}[0,1]$ there exists $f \in\left(V_{2} \backslash V_{2}^{0}\right) \cap C[0,1]$ such that $\mu_{f}=\mu$.

The solution of Problem 3 heavily relies on properties of the measure $\mu_{f}$. In particular the following inequality is a key ingredient. For every $f \in V_{2}$ the following holds:

$$
\sqrt{\left\|\mu_{f}\right\|} \leq \operatorname{dist}\left(f, V_{2}^{0}\right) \leq\left\|\widetilde{o s} \mathcal{K}_{\mathcal{K}} f\right\|_{\infty} \leq \sqrt{\left\|\mu_{f}\right\|}+2 \sqrt{\left\|\mu_{f}^{d}\right\|}
$$

where $\mathcal{K}$ is a w*-closed subset of $B_{\left(V_{2}^{0}\right)^{*}}$, 1-norming $V_{2}^{0}, \widetilde{o s c} f$ is as in [21] or [2] and was introduced in [13] and also $\mu_{f}^{d}$ is the discrete part of $\mu_{f}$. Note that when $f$ is continuous, then (10) becomes an equality and hence $\operatorname{dist}\left(f, V_{2}^{0}\right)=\sqrt{\left\|\mu_{f}\right\|}$. Furthermore the measures $\left\{\mu_{f}: f \in V_{2}\right\}$ permit us to have a better and more precise understanding of the structure of $X$ when $X^{* *}$ is non-separable. Thus we prove the following.

Theorem. Let $X$ be a closed subspace of $V_{2}^{0}$. Then the following hold.

(1) The space $X$ contains an isomorphic copy of $c_{0}$ if and only if $X^{* *}$ is nonseparable.

(2) The space $X$ contains an isomorphic copy of $S^{2}$ if and only if $\mathcal{M}_{X^{* *}}=$ $\left\{\mu_{f}: f \in X^{* *}\right\}$ is a non-separable subset of $\mathcal{M}[0,1]$.

Note that when $X^{*}$ is non-separable, then the stronger case (case (2)) of the above theorem occurs. When $X$ is isomorphic to $c_{0}$, then both $X^{*}$ and $\mathcal{M}_{X^{* *}}$ are separable. On the other hand, any subspace $X$ of $V_{2}^{0}$ isomorphic to $S^{2}$ is an example of a subspace $X$ with separable dual and such that $\mathcal{M}_{X^{* *}}$ is non-separable.

In the rest of the introduction we shall describe the basic steps towards a proof of the main theorem. Let us start by saying that a function $h \in V_{2}^{0}$ is $(C, \varepsilon)$-dominated by a measure $\mu \in \mathcal{M}^{+}[0,1]$, if for every finite family $\mathcal{I}=$ $\left(\left[a_{i}, b_{i}\right]\right)_{i=1}^{n}$ of non-overlapping intervals it follows that

$$
\sum_{i=1}^{n}\left(h\left(b_{i}\right)-h\left(a_{i}\right)\right)^{2} \leq C \mu(\cup \mathcal{I})+\varepsilon .
$$


This domination property allows us to relate measures with sequences $\left(h_{n}\right)_{n}$ which are equivalent to the usual basis of $c_{0}$ as follows.

Proposition 3. Let $\left(h_{n}\right)_{n}$ be a seminormalized sequence of functions of $V_{2}^{0},\left(\varepsilon_{n}\right)_{n}$ be a null sequence of positive real numbers and $\mu \in \mathcal{M}^{+}[0,1]$ such that for some $C>0$ each $h_{n}$ is $\left(C, \varepsilon_{n}\right)-\mu$ dominated and $\lim _{n}\left\|h_{n}\right\|_{\infty}=0$. Then there is a subsequence of $\left(h_{n}\right)_{n}$ equivalent to the usual basis of $c_{0}$.

The next result explains how we can extract information about $X$ from the elements of $X^{* *} \backslash X$.

Proposition 4. Let $X$ be a subspace of $V_{2}^{0}, f \in X^{* *} \backslash X$ and $\left(f_{n}\right)_{n}$ be a bounded sequence in $X$ pointwise convergent to $f$. Then for every $0<\delta<\operatorname{dist}(f, X)$ and every sequence $\left(\varepsilon_{n}\right)_{n}$ of positive real numbers there exists a convex block sequence $\left(h_{n}\right)_{n}$ of $\left(f_{n}\right)_{n}$ such that for all $n<m$ the following properties are satisfied.

(i) $\delta<\left\|h_{m}-h_{n}\right\|_{V_{2}} \leq 2 M$, where $M=\sup _{n}\left\|f_{n}\right\|_{V_{2}}$.

(ii) $\left\|h_{m}-h_{n}\right\|_{\infty} \leq 2\|\widetilde{o s c}[0,1] f\|_{\infty}+\varepsilon_{n} \leq 4\|f\|_{\infty}+\varepsilon_{n}$.

(iii) $h_{m}-h_{n}$ is $\left(4, \widetilde{\varepsilon}_{n}\right)-\mu_{f}$ dominated, where $\widetilde{\varepsilon}_{n}=32\|f\|_{V_{2}} \sqrt{\left\|\mu_{f}^{d}\right\|}+\varepsilon_{n}$.

The proof of the proposition uses inequality (11) and also optimal sequences pointwise convergent to the function $f$ (cf. 2]). Note that if we additionally assume that $f$ is continuous, in which case $\mu_{f}^{d}=\widetilde{o s c}_{[0,1]} f=0$, Propositions 3 and 4 almost immediately yield that the space $X$ contains $c_{0}$, a result initially proved with a different method in 3 .

The proof of the main result is divided into two cases. In the first case we consider subspaces $X$ of $V_{2}^{0}$ with $X^{*}$ separable, $X^{* *}$ non-separable and $\mathcal{M}_{X^{* *}}$ separable. Then using Proposition 4 and the separability of $X^{*}$, we may select a seminormalized sequence $\left(H_{n}\right)_{n}$ in $X$ and a norm-converging sequence of measures $\left(\mu_{n}\right)_{n}$ in $\mathcal{M}_{X^{* *}}$ such that each $H_{n}$ is $\left(4, \varepsilon_{n}\right)-\mu_{n}$ dominated. Then an easy modification of Proposition 3 yields that there exists a subsequence of $\left(H_{n}\right)_{n}$ equivalent to the $c_{0}$-basis.

The second case, namely when $\mathcal{M}_{X^{* *}}$ is non-separable, is more involved. Here, we first give sufficient conditions for the embedding of the space $S^{2}$ into a subspace $X$ of $V_{2}^{0}$. Moreover, again using Proposition 4, we construct a seminormalized tree family of functions $\left(H_{s}\right)_{s \in 2<\mathbb{N}}$ in $X$ and a bounded family of measures $\left(\mu_{s}\right)_{s \in 2<\mathbb{N}}$. For each $s \in 2^{n}$ we define a finite subset $L_{s} \subseteq 2^{2 n}$ with $\operatorname{card}\left(L_{s}\right)=2^{n}$ and we set $G_{s}=2^{-n / 2} \sum_{t \in L_{s}} H_{t}$ and $\nu_{s}=2^{-n} \sum_{t \in L_{s}} \mu_{t}$. The proof ends by showing that these new tree families satisfy the requirements for containing a tree subfamily equivalent to the $S^{2}$-basis.

The present work can be considered as a step towards the understanding of the structure of $V_{2}^{0}$. Our approach has revealed a new component, the Hausdorff measure $\mu_{f}$ associated to a function $f$ of bounded quadratic variation, which is of independent interest and could be useful to a further investigation of $V_{2}$ as well as in applications.

We close this introduction by pointing out that all the results contained here remain valid under obvious modifications for the space $V_{p}^{0}$, for all $1<p<\infty$.

\section{Preparatory work on $V_{2}$}

This section is divided into three subsections. First we fix the notation that we shall use. In the second subsection we prove that the set of discontinuity points 
of the elements of $X^{* *}$ when $X^{*}$ is separable is countable and also in this case $\left(X^{*},\|\cdot\|_{\infty}\right)$ is separable. Finally, we introduce the biorthogonal families of functions of $V_{2}^{0}$. Such families share nice properties and as we will see they play a critical role in the proofs of almost all of our results.

2.1. Preliminaries. We start with some notation concerning intervals as well as families of intervals of $[0,1]$. The length of an interval $I$ will be denoted by $|I|$. For a finite family $\mathcal{I}$ of intervals, $\|\mathcal{I}\|_{\max }=\max \{|I|: I \in \mathcal{I}\}$ and $\|\mathcal{I}\|_{\min }=\min \{|I|:$ $I \in \mathcal{I}\}$.

By $\mathcal{A}$ we denote the set of all finite families of intervals of $[0,1]$ with pairwise disjoint interiors. A sequence $\left(\mathcal{I}_{i}\right)_{i}$ in $\mathcal{A}$ will be called disjoint if for every $i \neq j$, $I \in \mathcal{I}_{i}$ and $J \in \mathcal{I}_{j}$, the interiors of $I$ and $J$ are disjoint. Also by $\mathcal{F}$ we denote the set of all finite families of pairwise disjoint closed intervals of $[0,1]$. More generally, given a subset $S \subseteq[0,1], \mathcal{F}(S)$ is the set of all $\mathcal{I} \in \mathcal{F}$ such that the endpoints of every $I \in \mathcal{I}$ belong to $S$.

Given $f:[0,1] \rightarrow \mathbb{R}$ and $\mathcal{P}=\left\{t_{0}<\ldots<t_{p}\right\} \subseteq[0,1]$, with $p \geq 1$, the quadratic variation of $f$ on $\mathcal{P}$ is the quantity

$$
v_{2}(f, \mathcal{P})=\left(\sum_{i=0}^{p-1}\left(f\left(t_{i+1}\right)-f\left(t_{i}\right)\right)^{2}\right)^{1 / 2} .
$$

Similarly for $\mathcal{I}=\left(I_{i}\right)_{i=1}^{k}$ in $\mathcal{A}$, we set $v_{2}(f, \mathcal{I})=\left(\sum_{i=0}^{k}\left(f\left(b_{i}\right)-f\left(a_{i}\right)\right)^{2}\right)^{1 / 2}$, where for each $1 \leq i \leq k, a_{i}, b_{i}$ are the endpoints of $I_{i}$ (if $\mathcal{I}$ is the empty sequence, then we define $\left.v_{2}(f, \varnothing)=0\right)$. The quantity $v_{2}(f, \mathcal{I})$ has also been defined in 19 where the exponent $1 / 2$ is omitted.

Notice that every $\mathcal{P}$ as above determines the family $\mathcal{I}_{\mathcal{P}}=\left(\left(t_{i}, t_{i+1}\right)\right)_{i=0}^{p-1}$ in $\mathcal{A}$ and $v_{2}(f, \mathcal{P})=v_{2}\left(f, \mathcal{I}_{\mathcal{P}}\right)$. It is easy to see that for every $f, g:[0,1] \rightarrow \mathbb{R}$ and every $\mathcal{I} \in \mathcal{A}$, we have that

$$
\left|v_{2}(f, \mathcal{I})-v_{2}(g, \mathcal{I})\right| \leq v_{2}(f+g, \mathcal{I}) \leq v_{2}(f, \mathcal{I})+v_{2}(g, \mathcal{I}) .
$$

Moreover for every disjoint partition $\mathcal{I}=\bigcup_{i} \mathcal{I}_{i}$ of $\mathcal{I} \in \mathcal{A}$,

$$
v_{2}(f, \mathcal{I}) \leq \sum_{i} v_{2}\left(f, \mathcal{I}_{i}\right) \quad \text { and } \quad v_{2}^{2}(f, \mathcal{I})=\sum_{i} v_{2}^{2}\left(f, \mathcal{I}_{i}\right)
$$

For $\widetilde{\mathcal{I}}, \mathcal{I}$ in $\mathcal{A}$, we write $\widetilde{\mathcal{I}} \preceq \mathcal{I}$ if for every $\widetilde{I} \in \widetilde{\mathcal{I}}$ there is $I \in \mathcal{I}$ such that $\widetilde{I} \subseteq I$.

For every $\varepsilon>0, D \subseteq[0,1]$ and $H_{1}, \ldots, H_{k}$ in $V_{2}^{0}$ we will say that $D \varepsilon$-determines the quadratic variation of the linear span $\left\langle H_{1}, \ldots, H_{k}\right\rangle$ if for every $\mathcal{I} \in \mathcal{A}$ there is $\widetilde{\mathcal{I}} \preceq \mathcal{I}$ in $\mathcal{F}(D)$ such that

$$
\left|v_{2}^{2}\left(\sum_{i=1}^{k} \lambda_{i} H_{i}, \mathcal{I}\right)-v_{2}^{2}\left(\sum_{i=1}^{k} \lambda_{i} H_{i}, \widetilde{\mathcal{I}}\right)\right| \leq\left(\sum_{i=1}^{k}\left|\lambda_{i}\right|^{2}\right) \varepsilon,
$$

for every sequence of scalars $\left(\lambda_{i}\right)_{i=1}^{k}$. Using standard approximation arguments the following is easily proved.

Proposition 1. Let $k \in \mathbb{N}, H_{1}, \ldots, H_{k}$ in $V_{2}^{0}$ and $\varepsilon>0$. Then there exists $\delta>0$ such that every $D \subseteq[0,1]$ which is $\delta$-dense in $[0,1] \varepsilon$-determines the quadratic variation of $\left\langle H_{1}, \ldots, H_{k}\right\rangle$.

Next we state some notation for the dyadic tree. For every $n \geq 0$, we set $2^{n}=\{0,1\}^{n}$ (where $2^{0}=\{\emptyset\}$ ). Hence for $n \geq 1$, every $s \in 2^{n}$ is of the form 
$s=(s(1), \ldots, s(n))$. For $0 \leq m<n$ and $s \in 2^{n}, s \mid m=(s(1), \ldots, s(m))$, where if $m=0, s \mid 0=\emptyset$. Also, $2^{\leqslant n}=\bigcup_{i=0}^{n} 2^{i}$ and $2^{<\mathbb{N}}=\bigcup_{n=0}^{\infty} 2^{n}$. The length $|s|$ of an $s \in 2^{<\mathbb{N}}$ is the unique $n \geq 0$ such that $s \in 2^{n}$. The initial segment partial ordering on $2^{<\mathbb{N}}$ will be denoted by $\sqsubseteq$ (i.e. $s \sqsubseteq t$ if $m=|s| \leq|t|$ and $s=t \mid m$ ). For $s, t \in 2^{<\mathbb{N}}$, $s \perp t$ means that $s, t$ are $\sqsubseteq$-incomparable (that is, neither $s \sqsubseteq t$ nor $t \sqsubseteq s$ ). For an $s \in 2^{<\mathbb{N}}, s^{\frown} 0$ and $s^{\frown} 1$ denote the two immediate successors of $s$ which end with 0 and 1 , respectively. More generally for $s, u \in 2^{<\mathbb{N}}, s^{\wedge} u$ denotes the concatenation of $s$ and $u$, namely the element $t \in 2^{<\mathbb{N}}$ with $|t|=|s|+|u|, t(i)=s(i)$ for all $1 \leq i \leq|s|$ and $t(|s|+i)=u(i)$ for all $1 \leq i \leq|u|$.

An antichain of $2^{<\mathbb{N}}$ is a subset of $2^{<\mathbb{N}}$ such that for every $s, t \in A, s \perp t$. A branch of $2^{<\mathbb{N}}$ is a maximal totally ordered subset of $2^{<\mathbb{N}}$. A dyadic subtree is a subset $T$ of $2^{<\mathbb{N}}$ such that there is an order isomorphism $\phi: 2^{<\mathbb{N}} \rightarrow T$. In this case $T$ is denoted by $T=\left(t_{s}\right)_{s \in 2<\mathbb{N}}$, where $t_{s}=\phi(s)$.

In the sequel by the term subspace we always mean a closed infinite-dimensional subspace. We also use the standard notation for Banach spaces from [16].

2.2. The discontinuities of $X^{* *}$ for subspaces $X$ of $V_{2}^{0}$. For every $f \in V_{2}$, by $D_{f}$ we denote the set of all points of discontinuity of $f$. For all $t \in[0,1]$ let $f\left(t^{+}\right)=\lim _{s \rightarrow t^{+}} f(s)$ and $f\left(t^{-}\right)=\lim _{s \rightarrow t^{-}} f(s)$ (where by convention we set $f\left(0^{-}\right)=f(0)$ and $f\left(1^{+}\right)=f(1)$ ). It is easily shown that for every $f \in V_{2}$, the set $D_{f}$ is at most countable and so $f$ is a Baire- 1 function. Moreover for every $t \in D_{f}$, $f\left(t^{-}\right)$and $f\left(t^{+}\right)$always exist and $\sum_{t \in D_{f}}\left|f(t)-f\left(t^{-}\right)\right|^{2}+\left|f(t)-f\left(t^{+}\right)\right|^{2} \leq\|f\|_{V_{2}}^{2}$.

In this subsection we will study the set $D_{X^{* *}}=\bigcup_{f \in X^{* *}} D_{f}$, for subspaces $X$ of $V_{2}^{0}$ with $X^{*}$ separable and $X^{* *}$ non-separable. We will show that $D_{X^{* *}}$ is a countable subset of $[0,1]$ which as we will see implies that the space $\left(X^{* *},\|\cdot\|_{\infty}\right)$ is separable. We start with a characterization of the subspaces $X$ of $V_{2}^{0}$ with separable dual through the discontinuity points of all $f \in X^{* *}$.

Proposition 2. Let $X$ be a subspace of $V_{2}^{0}$. Then $X^{*}$ is separable if and only if $D_{X^{* *}}$ is countable.

Proof. Suppose that $D_{X^{* *}}$ is uncountable. Then, since for every $f \in X^{* *}, D_{f}$ is countable, we may choose uncountable sets $\mathcal{F}=\left\{f_{\xi}\right\}_{\xi<\omega_{1}} \subseteq B_{X^{* *}}$ and $A=$ $\left\{t_{\xi}\right\}_{\xi<\omega_{1}} \subseteq[0,1]$ such that the following are satisfied.

(1) For every $\xi<\omega_{1}, f_{\xi}$ is discontinuous at $t_{\xi}$.

(2) Exactly one of the following hold.

(2a) For all $\xi<\omega_{1}, f_{\xi}\left(t_{\xi}^{+}\right) \neq f_{\xi}\left(t_{\xi}\right)$.

(2b) For all $\xi<\omega_{1}, f_{\xi}\left(t_{\xi}^{-}\right) \neq f_{\xi}\left(t_{\xi}\right)$.

Suppose that (2a) holds (the other case is similar). Passing to an uncountable subset of $\mathcal{F}$ we may assume that there exists $\delta>0$ such that $\left|f_{\xi}\left(t_{\xi}^{+}\right)-f_{\xi}\left(t_{\xi}\right)\right|>\delta$, for every $\xi<\omega_{1}$. Moreover, by passing to a further uncountable subset, we can suppose that there exist $0<\varepsilon<\delta$ and an open interval $I$ of $(0,1)$ such that for every $\xi<\omega_{1}$ we have that (i) $t_{\xi} \in I$, (ii) for every $t \in I$ and $t<t_{\xi},\left|f_{\xi}(t)-f_{\xi}\left(t_{\xi}^{-}\right)\right|<\varepsilon$ and (iii) for every $t \in I$ and $t>t_{\xi},\left|f_{\xi}(t)-f_{\xi}\left(t_{\xi}^{+}\right)\right|<\varepsilon$.

Let $\xi<\xi^{\prime}$. If $t_{\xi}<t_{\xi^{\prime}}$, then we have that

$$
\left|\delta_{t_{\xi}}\left(f_{\xi}\right)-\delta_{t_{\xi^{\prime}}}\left(f_{\xi}\right)\right| \geq\left|f_{\xi}\left(t_{\xi}\right)-f_{\xi}\left(t_{\xi}^{+}\right)\right|-\left|f_{\xi}\left(t_{\xi}^{+}\right)-f_{\xi}\left(t_{\xi^{\prime}}\right)\right|>\delta-\varepsilon,
$$

and if $t_{\xi^{\prime}}<t_{\xi}$, then similarly $\left|\delta_{t_{\xi}}\left(f_{\xi^{\prime}}\right)-\delta_{t_{\xi^{\prime}}}\left(f_{\xi^{\prime}}\right)\right|>\delta-\varepsilon$. 
This implies that $\left\|\left.\delta_{t_{\xi}}\right|_{X}-\left.\delta_{t_{\xi^{\prime}}}\right|_{X}\right\| \geq \delta-\varepsilon$ for every $\xi \neq \xi^{\prime}$ and therefore $X^{*}$ is non-separable. Finally for the converse, suppose that $X^{*}$ is non-separable. Then by Proposition 23 of [1] we have that $X^{* *}$ contains a non-separable family $\mathcal{H} \subseteq$ $V_{2}^{d}=\overline{\left\langle\left\{\chi_{t}: t \in(0,1)\right\}\right\rangle}$ and therefore $D_{X^{* *}}$ must be uncountable.

Let us recall that by [1] (Theorem 15), we have that

$$
V_{2}=\overline{V_{2}^{c}+\left\langle\left\{\chi_{[t, 1]}: 0<t \leq 1\right\}\right\rangle+\left\langle\left\{\chi_{t}: 0<t<1\right\}\right\rangle} \|^{\| \cdot V_{2}},
$$

where $V_{2}^{c}=V_{2} \cap C[0,1]$. The proof of this result is based on a series of lemmas (Lemmas 16-20). It is easy to see that the exact content of their proof is the following stronger result.

Lemma 3. For every $f \in V_{2}$, we have that

$$
f \in{\overline{V_{2}^{c}+\left\langle\left\{\chi_{[t, 1]}: t \in D_{f}\right\}\right\rangle+\left\langle\left\{\chi_{t}: t \in D_{f}\right\}\right\rangle}}^{\|\cdot\|_{V_{2}}} .
$$

Proposition 4. Let $\mathcal{F}$ be a subset of $V_{2}$ such that $D_{\mathcal{F}}=\bigcup_{f \in \mathcal{F}} D_{f}$ is countable. Then the space $\left(\mathcal{F},\|\cdot\|_{\infty}\right)$ is separable.

Proof. Lemma 3 readily yields that

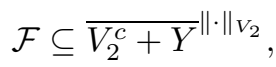

where $Y=\left\langle\left\{\chi_{[t, 1]}: t \in D_{\mathcal{F}}\right\}\right\rangle+\left\langle\left\{\chi_{t}: t \in D_{\mathcal{F}}\right\}\right\rangle$. Using that $\|\cdot\|_{\infty} \leq\|\cdot\|_{V_{2}}$, we get

$$
\mathcal{F} \subseteq{\overline{V_{2}^{c}+Y}}^{\|\cdot\|_{V_{2}}} \subseteq{\overline{V_{2}^{c}+Y}}^{\|\cdot\|_{\infty}} \subseteq \overline{C[0,1]+Y}^{\|\cdot\|_{\infty}} .
$$

Since $D_{\mathcal{F}}$ is countable the space $\left(Y,\|\cdot\|_{\infty}\right)$ is separable. Hence $\overline{C[0,1]+Y}\|\cdot\|_{\infty}$ is also separable which by (4) yields the desired result.

Corollary 5. Let $X$ be a subspace of $V_{2}^{0}$ such that $X^{*}$ is separable. Then the space $\left(X^{* *},\|\cdot\|_{\infty}\right)$ is separable.

\subsection{Biorthogonal families in $V_{2}^{0}$.}

2.3.1. Definition and existence. In this subsection we introduce the concept of biorthogonality for families of functions in $V_{2}^{0}$.

Definition 6. Let $\left(H_{i}\right)_{i \in S}$ be a family of functions of $V_{2}^{0}$ and $\left(\varepsilon_{i}\right)_{i \in S}$ a family of positive real numbers, where $S$ is a countable set. We will say that $\left(H_{i}\right)_{i \in S}$ is $\left(\varepsilon_{i}\right)_{i \in S}$-biorthogonal if for every $\mathcal{I} \in \mathcal{A}$ there is a disjoint partition $\mathcal{I}=\bigcup_{i \in S} \mathcal{I}^{(i)}$ such that for every $j \in S$,

$$
\sum_{\{i \in S: i \neq j\}} v_{2}\left(H_{i}, \mathcal{I}^{(j)}\right) \leq \varepsilon_{j} .
$$

Proposition 7. Let $\left(H_{n}\right)_{n \in \mathbb{N}}$ be a sequence of functions of $V_{2}^{0}$ with $\lim \left\|H_{n}\right\|_{\infty}=0$. Then for every sequence $\left(\varepsilon_{i}\right)_{i \in \mathbb{N}}$ of positive real numbers there exists a subsequence $\left(H_{n_{i}}\right)_{i \in \mathbb{N}}$ such that $\left(H_{n_{i}}\right)_{i \in \mathbb{N}}$ is $\left(\varepsilon_{i}\right)_{i \in \mathbb{N}}$-biorthogonal.

For the proof of the above proposition we will need some specialized forms of biorthogonality. Let $k \geq 1,\left(\varepsilon_{i}\right)_{i=1}^{k}$ and $\left(\delta_{i}\right)_{i=0}^{k-1}$ be finite sequences of positive real numbers such that $0<\delta_{k-1}<\ldots<\delta_{1}<\delta_{0}=1$. We say that a sequence $\left(H_{i}\right)_{i=1}^{k}$ in $V_{2}^{0}$ is $\left(\left(\varepsilon_{i}\right)_{i=1}^{k},\left(\delta_{i}\right)_{i=0}^{k-1}\right)$-biorthogonal if the inequality (5) of Definition 6 is satisfied 
for $S=\{1, \ldots, k\}$ and

$$
\mathcal{I}^{(j)}=\left\{I \in \mathcal{I}: \delta_{j}<|I| \leq \delta_{j-1}\right\}
$$

for all $1 \leq j \leq k$ (where for $j=k$, we set $\delta_{k}=0$ ).

We will also use the following notation. For a sequence of positive real numbers $\left(\varepsilon_{i}\right)_{i \in \mathbb{N}}$ and every $1 \leq i \leq k$, we set $\varepsilon_{i}^{k}=\left(\sum_{r=1}^{k-i+1} 2^{-r}\right) \varepsilon_{i}$. Clearly for all $1 \leq i \leq k$, $\varepsilon_{i}^{k}<\varepsilon_{i}^{k+1}$ and $\lim _{k} \varepsilon_{i}^{k}=\varepsilon_{i}$.

The proof of Proposition 7 is based on the next lemma.

Lemma 8. Let $\left(H_{i}\right)_{i=1}^{k}$ be an $\left(\left(\varepsilon_{i}^{k}\right)_{i=1}^{k},\left(\delta_{i}\right)_{i=0}^{k-1}\right)$-biorthogonal sequence of $V_{2}^{0}$. Let $0<\delta_{k}<\delta_{k-1}$ be such that for every $\mathcal{I} \in \mathcal{A}$ with $\|\mathcal{I}\|_{\max } \leq \delta_{k}$, the following holds:

$$
\sum_{i=1}^{k} v_{2}\left(H_{i}, \mathcal{I}\right) \leq \frac{\varepsilon_{k+1}}{2} .
$$

Then there is $\epsilon>0$ such that for every $H_{k+1} \in V_{2}^{0}$ with $\left\|H_{k+1}\right\|_{\infty}<\epsilon$, the sequence $\left(H_{i}\right)_{i=1}^{k+1}$ is $\left(\left(\varepsilon_{i}^{k+1}\right)_{i=1}^{k+1},\left(\delta_{i}\right)_{i=0}^{k}\right)$-biorthogonal.

Proof. Notice that for every $\mathcal{J} \in \mathcal{A}$ with $\delta_{k}<\|\mathcal{J}\|_{\min }, \operatorname{card}(\mathcal{J})<\delta_{k}^{-1}$. Set $\epsilon=\sqrt{\delta_{k}} 2^{-(k+3)} \min \left\{\varepsilon_{i}\right\}_{i=1}^{k}$ and let $H_{k+1} \in V_{2}^{0}$ be such that $\left\|H_{k+1}\right\|_{\infty}<\epsilon$. Then for every $1 \leq j \leq k$ and $\mathcal{I} \in \mathcal{A}$, we have that $\delta_{k}<\delta_{j}<\left\|\mathcal{I}^{(j)}\right\|_{\text {min }}$ and therefore

$$
v_{2}\left(H_{k+1}, \mathcal{I}^{(j)}\right) \leq\left(\delta_{k}^{-1}\left(2\left\|H_{k+1}\right\|_{\infty}\right)^{2}\right)^{1 / 2} \leq \frac{\min \left\{\varepsilon_{i}\right\}_{i=1}^{k}}{2^{k+2}} \leq \frac{\varepsilon_{j}}{2^{k-j+2}} .
$$

Hence for each $1 \leq j \leq k$ and for every $\mathcal{I} \in \mathcal{A}$,

$$
\begin{aligned}
\sum_{\{i: 1 \leq i \leq k+1, i \neq j\}} v_{2}\left(H_{i}, \mathcal{I}^{(j)}\right) & =\sum_{\{i: 1 \leq i \leq k, i \neq j\}} v_{2}\left(H_{i}, \mathcal{I}^{(j)}\right)+v_{2}\left(H_{k+1}, \mathcal{I}^{(j)}\right) \\
& \leq \varepsilon_{j}^{k}+\frac{\varepsilon_{j}}{2^{k-j+2}}=\left(\sum_{r=1}^{k-j+2} 2^{-r}\right) \varepsilon_{j}=\varepsilon_{j}^{k+1} .
\end{aligned}
$$

Finally $\left\|\mathcal{I}^{(k+1)}\right\|_{\max } \leq \delta_{k}$ and so by (7), we get that

$$
\sum_{i=1}^{k} v_{2}\left(H_{i}, \mathcal{I}^{(k+1)}\right) \leq \frac{\varepsilon_{k+1}}{2}=\varepsilon_{k+1}^{k+1} .
$$

Proof of Proposition 7. We inductively construct an increasing sequence $n_{1}<n_{2}<$ ... of natural numbers and a decreasing sequence of positive real numbers $0<$ $\ldots<\delta_{2}<\delta_{1}<1=\delta_{0}$, such that for every $k \geq 1$, the sequence $\left(H_{n_{i}}\right)_{i=1}^{k}$ is $\left(\left(\varepsilon_{i}^{k}\right)_{i=1}^{k},\left(\delta_{i}\right)_{i=1}^{k-1}\right)$-biorthogonal. We claim that $\left(H_{n_{i}}\right)_{i}$ is $\left(\varepsilon_{i}\right)_{i}$-biorthogonal. Indeed, let $\mathcal{I} \in \mathcal{A}$ and let $\mathcal{I}=\bigcup_{i} \mathcal{I}^{(i)}$ be the partition of $\mathcal{I}$ induced by (6). Also let $k_{0} \geq 1$ be such that $\delta_{k_{0}}<\|\mathcal{I}\|_{\min }$. Then for each $j \in \mathbb{N}$ with $j \geq k_{0}, \mathcal{I}^{(j)}=\emptyset$ and so (5) trivially holds. Otherwise for all $k \geq 1, \sum_{\{1 \leq i \leq k: i \neq j\}} v_{2}\left(H_{n_{i}}, \mathcal{I}^{(j)}\right)<\varepsilon_{j}^{k}$ and so $\sum_{\{i \neq j\}} v_{2}\left(H_{n_{i}}, \mathcal{I}^{(j)}\right) \leq \varepsilon_{j}$ 
We will also need the analogue of the above in the case where $S=2^{<\mathbb{N}}$. We omit the proof since it is an easy modification of the one of Proposition 7 .

Proposition 9. Let $\left(H_{s}\right)_{s \in 2^{<N \mathbb{N}}}$ be a family of functions in $V_{2}^{0}$ such that for every $\sigma \in 2^{\mathbb{N}}, \lim _{n}\left\|H_{\sigma \mid n}\right\|_{\infty}=0$. Then for every family $\left(\varepsilon_{s}\right)_{s \in 2^{<\mathbb{N}}}$ of positive real

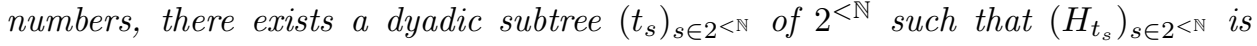

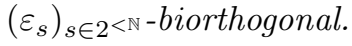

2.3.2. Estimations on biorthogonal sequences. In the next two lemmas and proposition, $S$ stands for a countable set and $\left(H_{i}\right)_{i \in S}$ is an $\left(\varepsilon_{i}\right)_{i \in S}$-biorthogonal family in $V_{2}^{0}$ such that $\sum_{i \in S} \varepsilon_{i}=\varepsilon<\infty$.

Lemma 10. Let $\mathcal{I} \in \mathcal{A}, F \subseteq S$ be finite and $\left(\lambda_{i}\right)_{i \in F}$ be a sequence of real numbers. Also let $\left(\mathcal{I}^{(i)}\right)_{i \in S}$ be a disjoint partition of $\mathcal{I}$ such that for every $j \in S$, $\sum_{\{i \in S: i \neq j\}} v_{2}\left(H_{i}, \mathcal{I}^{(j)}\right) \leq \varepsilon_{j}$ (see Definition [6). Then

(i) For every $i \in S, v_{2}\left(H_{i}, \mathcal{I} \backslash \mathcal{I}^{(i)}\right)<\varepsilon$.

(ii) For every $j \notin F, v_{2}\left(\sum_{i \in F} \lambda_{i} H_{i}, \mathcal{I}^{(j)}\right) \leq \max _{i \in F}\left|\lambda_{i}\right| \varepsilon_{j}$.

(iii) For every $j \in F, v_{2}\left(\sum_{i \in F} \lambda_{i} H_{i}, \mathcal{I}^{(j)}\right) \leq\left|\lambda_{j}\right| v_{2}\left(H_{j}, \mathcal{I}^{(j)}\right)+\max _{i \in F}\left|\lambda_{i}\right| \varepsilon_{j}$.

(iv) For every $j \in F, v_{2}\left(\sum_{i \in F} \lambda_{i} H_{i}, \mathcal{I}^{(j)}\right) \geq|| \lambda_{j}\left|v_{2}\left(H_{j}, \mathcal{I}^{(j)}\right)-\max _{i \in F}\right| \lambda_{i}\left|\varepsilon_{j}\right|$.

Proof. (i) Let $i \in S$. Then

$$
v_{2}\left(H_{i}, \mathcal{I} \backslash \mathcal{I}^{(i)}\right)=v_{2}\left(H_{i}, \bigcup_{j \neq i} \mathcal{I}^{(j)}\right) \leq \sum_{j \neq i} v_{2}\left(H_{i}, \mathcal{I}^{(j)}\right) \leq \sum_{j \neq i} \varepsilon_{j}<\varepsilon .
$$

(ii) Let $j \notin F$. Then

$$
v_{2}\left(\sum_{i \in F} \lambda_{i} H_{i}, \mathcal{I}^{(j)}\right) \leq \sum_{i \in F} v_{2}\left(\lambda_{i} H_{i}, \mathcal{I}^{(j)}\right)=\sum_{i \in F}\left|\lambda_{i}\right| v_{2}\left(H_{i}, \mathcal{I}^{(j)}\right) \leq \max _{i \in F}\left|\lambda_{i}\right| \varepsilon_{j} .
$$

(iii) Let $j \in F$. Then using (ii) we get that

$$
\begin{aligned}
v_{2}\left(\sum_{i \in F} \lambda_{i} H_{i}, \mathcal{I}^{(j)}\right) & \leq v_{2}\left(\lambda_{j} H_{j}, \mathcal{I}^{(j)}\right)+\sum_{i \neq j} v_{2}\left(\lambda_{i} H_{i}, \mathcal{I}^{(j)}\right) \\
& \leq\left|\lambda_{j}\right| v_{2}\left(H_{j}, \mathcal{I}^{(j)}\right)+\max _{i \in F}\left|\lambda_{i}\right| \varepsilon_{j} .
\end{aligned}
$$

(iv) Since $v_{2}\left(\sum_{i \in F} \lambda_{i} H_{i}, \mathcal{I}^{(j)}\right) \geq\left|v_{2}\left(\lambda_{j} H_{j}, \mathcal{I}^{(j)}\right)-\sum_{i \neq j} v_{2}\left(\lambda_{i} H_{i}, \mathcal{I}^{(j)}\right)\right|$, the proof is similar to that of (ii).

Lemma 11. Suppose that there is $M>0$ such that $\left\|H_{i}\right\|_{V_{2}} \leq M$, for all $i \in$ $S$. Let $\mathcal{I} \in \mathcal{A}, F \subseteq G \subseteq S$ be finite and $\left(\lambda_{i}\right)_{i \in G}$ be a sequence of real numbers. Also let $\left(\mathcal{I}^{(i)}\right)_{i \in S}$ be a disjoint partition of $\mathcal{I}$ such that for every $j \in S$, $\sum_{\{i \in S: i \neq j\}} v_{2}\left(H_{i}, \mathcal{I}^{(j)}\right) \leq \varepsilon_{j}$. Then the following are satisfied.

(i) $v_{2}^{2}\left(\sum_{i \in F} \lambda_{i} H_{i}, \mathcal{I}\right) \leq \sum_{i \in F}\left|\lambda_{i}\right|^{2} v_{2}^{2}\left(H_{i}, \mathcal{I}^{(i)}\right)+\max _{i \in F}\left|\lambda_{i}\right|^{2}(2 M+\varepsilon) \varepsilon$.

(ii) $v_{2}^{2}\left(\sum_{i \in G} \lambda_{i} H_{i}, \mathcal{I}\right)>\sum_{i \in F}\left|\lambda_{i}\right|^{2} v_{2}^{2}\left(H_{i}, \mathcal{I}^{(i)}\right)-\max _{i \in G}\left|\lambda_{i}\right|^{2} 2 M \varepsilon$.

(iii) $v_{2}^{2}\left(\sum_{i \in F} \lambda_{i} H_{i}, \mathcal{I}\right) \leq v_{2}^{2}\left(\sum_{i \in G} \lambda_{i} H_{i}, \mathcal{I}\right)+\max _{i \in G}\left|\lambda_{i}\right|^{2}(4 M+\varepsilon) \varepsilon$. 
Proof. (i) By (ii) and (iii) of Lemma 10, we have that

$$
\begin{aligned}
v_{2}^{2}\left(\sum_{i \in F} \lambda_{i} H_{i}, \mathcal{I}\right) & =\sum_{j \in F} v_{2}^{2}\left(\sum_{i \in F} \lambda_{i} H_{i}, \mathcal{I}^{(j)}\right)+\sum_{j \in S \backslash F} v_{2}^{2}\left(\sum_{i \in F} \lambda_{i} H_{i}, \mathcal{I}^{(j)}\right) \\
& \leq \sum_{j \in F}\left(\left|\lambda_{j}\right| v_{2}\left(H_{j}, \mathcal{I}^{(j)}\right)+\max _{i \in F}\left|\lambda_{i}\right| \varepsilon_{j}\right)^{2}+\sum_{j \in S \backslash F} \max _{i \in F}\left|\lambda_{i}\right|^{2} \varepsilon_{j}^{2} \\
& \leq \sum_{j \in F}\left|\lambda_{j}\right|^{2} v_{2}^{2}\left(H_{j}, \mathcal{I}^{(j)}\right)+\max _{j \in F}\left|\lambda_{j}\right|^{2}(2 M+\varepsilon) \varepsilon .
\end{aligned}
$$

(ii) Using (iv) of Lemma 10, we obtain that

$$
\begin{aligned}
& v_{2}^{2}\left(\sum_{i \in G} \lambda_{i} H_{i}, \mathcal{I}\right) \geq v_{2}^{2}\left(\sum_{i \in G} \lambda_{i} H_{i}, \bigcup_{j \in F} \mathcal{I}^{(j)}\right)=\sum_{j \in F} v_{2}^{2}\left(\sum_{i \in G} \lambda_{i} H_{i}, \mathcal{I}^{(j)}\right) \\
& \geq \sum_{j \in F}|| \lambda_{j}\left|v_{2}\left(H_{j}, \mathcal{I}^{(j)}\right)-\max _{i \in G}\right| \lambda_{i}\left|\varepsilon_{j}\right|^{2} \geq \sum_{j \in F}\left|\lambda_{j}\right|^{2} v_{2}^{2}\left(H_{j}, \mathcal{I}^{(j)}\right)-\max _{i \in G}\left|\lambda_{i}\right|^{2} 2 M \varepsilon .
\end{aligned}
$$

Finally (iii) follows easily from (i) and (ii).

Proposition 12. Let $M>\theta>2 \varepsilon>0$ and suppose that $\theta<\left\|H_{i}\right\|_{V_{2}} \leq M$, for all $i \in S$. Then $\left(H_{i}\right)_{i \in S}$ is an unconditional family.

Proof. Let $F \subseteq G$ be finite subsets of $S$ and let $\left|\lambda_{i_{0}}\right|=\max _{i \in G}\left|\lambda_{i}\right|$. By (iii) of Lemma 11, we easily get that

$$
\left\|\sum_{i \in F} \lambda_{i} H_{i}\right\|_{V_{2}}^{2} \leq\left\|\sum_{i \in G} \lambda_{i} H_{i}\right\|_{V_{2}}^{2}+\left|\lambda_{i_{0}}\right|^{2}(4 M+\varepsilon) \varepsilon .
$$

Let $\mathcal{I}_{0} \in \mathcal{A}$ such that $v_{2}\left(H_{i_{0}}, \mathcal{I}_{0}\right)>\theta$. Since $\left(H_{i}\right)_{i \in S}$ is $\left(\varepsilon_{i}\right)_{i \in S}$-biorthogonal, we get that there is a disjoint partition $\left(\mathcal{I}^{(i)}\right)_{i \in S}$ of $\mathcal{I}$ such that for every $j \in S$, $\sum_{\{i \in S: i \neq j\}} v_{2}\left(H_{i}, \mathcal{I}^{(j)}\right) \leq \varepsilon_{j}$. Then

$$
v_{2}\left(H_{i_{0}}, \mathcal{I}_{0}^{\left(i_{0}\right)}\right) \geq v_{2}\left(H_{i_{0}}, \mathcal{I}_{0}\right)-v_{2}\left(H_{i_{0}}, \mathcal{I} \backslash \mathcal{I}_{0}^{\left(i_{0}\right)}\right)>\theta-\varepsilon .
$$

Moreover by (ii) of Lemma 10, we have that

$$
\left|v_{2}\left(\lambda_{i_{0}} H_{i_{0}}, \mathcal{I}_{0}^{\left(i_{0}\right)}\right)-v_{2}\left(\sum_{i \in G} \lambda_{i} H_{i}, \mathcal{I}_{0}^{\left(i_{0}\right)}\right)\right| \leq v_{2}\left(\sum_{i \in G, i \neq i_{0}} \lambda_{i} H_{i}, \mathcal{I}_{0}^{\left(i_{0}\right)}\right) \leq\left|\lambda_{i_{0}}\right| \varepsilon,
$$

and so

$$
\left|\lambda_{i_{0}}\right| v_{2}\left(H_{i_{0}}, \mathcal{I}_{0}^{\left(i_{0}\right)}\right) \leq v_{2}\left(\sum_{i \in G} \lambda_{i} H_{i}, \mathcal{I}_{0}^{\left(i_{0}\right)}\right)+\left|\lambda_{i_{0}}\right| \varepsilon \leq\left\|\sum_{i \in G} \lambda_{i} H_{i}\right\|_{V_{2}}+\left|\lambda_{i_{0}}\right| \varepsilon .
$$

By (9) and (10), we get that

$$
\left|\lambda_{i_{0}}\right| \leq \frac{1}{\theta-2 \varepsilon}\left\|\sum_{i \in G} \lambda_{i} H_{i}\right\|_{V_{2}} .
$$

Hence by (8), we have that

$$
\left\|\sum_{i \in F} \lambda_{i} H_{i}\right\|_{V_{2}} \leq\left(1+\frac{(4 M+\varepsilon) \varepsilon}{(\theta-2 \varepsilon)^{2}}\right)^{1 / 2}\left\|\sum_{i \in G} \lambda_{i} H_{i}\right\|_{V_{2}}
$$

and the proof of the proposition is complete. 


\section{HausdorfF measures associated to FUnCtions OF BOUNDED QUADRATIC VARIATION}

The aim of this section is to introduce and study the fundamental properties of the measure $\mu_{f}$ corresponding to a function $f \in V_{2}$. It is divided into three subsections. The first includes the definition and initial properties of the measure $\mu_{f}$. The second is mainly devoted to the proof of Theorem 20, and the last contains a study of the points of non-differentiability of a function $f \in V_{2}$.

3.1. Definition and elementary properties. For every $f \in V_{2}$ and for every interval $I$ of $[0,1]$ we set

$$
\widetilde{\mu}_{f}(I)=\inf _{\delta>0} \widetilde{\mu}_{f, \delta}(I),
$$

where for each $\delta>0, \widetilde{\mu}_{f, \delta}(I)=\sup \left\{v_{2}^{2}(f, \mathcal{P}): \mathcal{P} \subseteq I\right.$ and $\left.\|\mathcal{P}\|_{\max }<\delta\right\}$.

We also define the function $\widetilde{F}_{f}: \mathbb{R} \rightarrow \mathbb{R}$ by

$$
\widetilde{F}_{f}(x)= \begin{cases}0, & \text { if } x \leq 0, \\ \widetilde{\mu}_{f}[0, x], & \text { if } 0<x<1, \\ \widetilde{\mu}_{f}[0,1], & \text { if } x \geq 1\end{cases}
$$

Notice that $\widetilde{F}_{f}$ is a non-negative increasing function on $\mathbb{R}$ and so taking the upper envelope $F_{f}(x)=\widetilde{F}_{f}\left(x^{+}\right)$of $\widetilde{F}_{f}$, we have that $F_{f}$ is in addition a right continuous function. Moreover since $\lim _{x \rightarrow-\infty} F_{f}(x)=0$ and $\lim _{x \rightarrow+\infty} F_{f}(x)=\widetilde{\mu}_{f}[0,1], F_{f}$ is the distribution function of a finite positive Borel measure on $\mathbb{R}$ which we will denote by $\mu_{f}$. Notice that $\mu_{f}=0$ if and only if $f \in V_{2}^{0}$ and also that $\mu_{f} \leq\|f\|_{V_{2}}$. Actually, it is easy to see that defining for any function $f:[0,1] \rightarrow \mathbb{R}$, the measure $\mu_{f}$ as above, then $\mu_{f}$ is finite if and only if $f \in V_{2}$. Since $\mu_{f}(-\infty, 0)=\mu_{f}(1,+\infty)=0$, in the sequel we will identify $\mu_{f}$ with its restriction on $[0,1]$.

Remark 1. The definition of the measure $\mu_{f}$ is generalized as follows. Let $(X, \rho)$ be a metric space and $f: X \rightarrow \mathbb{R}$ be a real-valued function. Following C.A. Rogers in [20], for a function $h:[0,+\infty] \rightarrow[0,+\infty]$ satisfying the conditions of p.50 of [20] and every open subset $G$ of $X$, we define the premeasure $h_{f}(G)=h(\operatorname{diam} f[G])$. Next following Method II (see [20]), we induce the measure $\mu_{f}^{h}$ defined on the Borel subsets of $X$. It is easy to see that in the case of $f \in V_{2}$ and for $h(x)=x^{2}$, the measure $\mu_{f}^{h}$ coincides with the measure $\mu_{f}$ defined above. Although the measures $\mu_{f}^{h}$ are not mentioned as Hausdorff measures in the literature, their definition and geometrical properties motivate us to include them in the latter class. It seems interesting to examine the regularity conditions that a function $f: X \rightarrow \mathbb{R}$ must satisfy so that the corresponding measure $\mu_{f}^{h}$ is a finite Borel measure. For example, this easily yields that $f$ has at most countably many discontinuities. Hence if $X$ is a Polish space, then $f$ is a Baire-1 function.

The following two lemmas are easily proved. The second one is essentially contained in [1] (Lemma 18).

Lemma 13. Let $0 \leq a<x<b \leq 1$ such that $f$ is continuous at $x$. Then for every $I=I_{1} \cup I_{2}$, where $I$ is an interval with endpoints $a, b$ and $I_{1}, I_{2}$ are intervals with $\sup I_{1}=x=\inf I_{2}$, we have that

$$
\widetilde{\mu}_{f}(I)=\widetilde{\mu}_{f}\left(I_{1}\right)+\widetilde{\mu}_{f}\left(I_{2}\right) .
$$


Lemma 14. (a) For every $x \in(0,1]$ and every $\varepsilon>0$ there exists $0<\delta<x$ such that $\sup \left\{v_{2}^{2}(f, \mathcal{P}): \mathcal{P} \subseteq[x-\delta, x)\right\} \leq \varepsilon$. In particular $\widetilde{\mu}_{f}[x-\delta, x) \leq \varepsilon$.

(b) Similarly for every $x \in[0,1)$ and every $\varepsilon>0$ there exists $0<\delta<1-x$ such that $\sup \left\{v_{2}^{2}(f, \mathcal{P}): \mathcal{P} \subseteq(x, x+\delta]\right\} \leq \varepsilon$. In particular $\widetilde{\mu}_{f}(x, x+\delta] \leq \varepsilon$.

Proposition 15. (a) $D_{f}=D_{F_{f}}=D_{\widetilde{F}_{f}}$ and so $f$ is continuous if and only if $\mu_{f}$ is continuous. Also for $x \in[0,1] \backslash D_{f}, \widetilde{F}_{f}(x)=F_{f}(x)$.

(b) If $f$ is continuous at $x$, then $\mu_{f}[0, x]=\widetilde{\mu}_{f}[0, x]$ and $\mu_{f}[x, 1]=\widetilde{\mu}_{f}[x, 1]$.

(c) For all continuity points $x<y$ of $f, \mu_{f}[x, y]=\widetilde{\mu}_{f}[x, y]$.

(d) For every open interval $(\alpha, \beta)$ of $[0,1], \mu_{f}(\alpha, \beta)=\widetilde{\mu}_{f}(\alpha, \beta)$.

Proof. (a) By the monotonicity of $\widetilde{F}_{f}$ we have that for all $x_{0} \in \mathbb{R}, \widetilde{F}_{f}\left(x_{0}^{+}\right)=F_{f}\left(x_{0}^{+}\right)$ and $\widetilde{F}_{f}\left(x_{0}^{-}\right)=F_{f}\left(x_{0}^{-}\right)$. Therefore $D_{F_{f}}=D_{\widetilde{F}_{f}}$ and for every $x_{0} \in[0,1] \backslash D_{\widetilde{F}_{f}}$, $\widetilde{F}_{f}\left(x_{0}\right)=F_{f}\left(x_{0}\right)$. It remains to show that $D_{f}=D_{\widetilde{F}_{f}}$. Let $x_{0} \in[0,1]$ be a continuity point of $f$. If $x_{0}<1$, by Lemma 13 we have that for every $x_{0}<y \leq 1$, $\widetilde{F}_{f}(y)-\widetilde{F}_{f}\left(x_{0}\right)=\widetilde{\mu}_{f}[0, y]-\widetilde{\mu}_{f}\left[0, x_{0}\right]=\widetilde{\mu}_{f}\left(x_{0}, y\right]$ and so by Lemma 14(b), $\widetilde{F}_{f}\left(x_{0}^{+}\right)=$ $\widetilde{F}_{f}\left(x_{0}\right)$. If $0<x_{0}$, again by Lemma 13, for every $0 \leq y<x_{0}$ such that $y$ is a continuity point of $f$ we have that $\widetilde{F}_{f}\left(x_{0}\right)-\widetilde{F}_{f}(y)=\widetilde{\mu}_{f}\left(y, x_{0}\right]$. Since $[0,1] \backslash D_{f}$ is dense in $[0,1]$, by Lemma 14(b) we get that $\widetilde{F}_{f}$ is continuous at $x_{0}$. Conversely suppose that $x_{0} \in D_{f}$. Then either $f\left(x_{0}^{+}\right) \neq f\left(x_{0}\right)$ or $f\left(x_{0}^{-}\right) \neq f\left(x_{0}\right)$. Suppose that $f\left(x_{0}^{+}\right) \neq f\left(x_{0}\right)$ (the other case is similarly treated). Then it is easy to see that for every $0<\delta<1-x_{0}, \widetilde{\mu}_{f}\left[x_{0}, x_{0}+\delta\right] \geq\left|f\left(x_{0}^{+}\right)-f\left(x_{0}\right)\right|^{2}$ and using Lemma 13, we obtain that

$$
\begin{aligned}
\widetilde{F}_{f}\left(x_{0}^{+}\right)=\lim _{\delta \rightarrow 0} \widetilde{F}_{f}\left(x_{0}+\delta\right) & \geq \lim _{\delta \rightarrow 0}\left(\widetilde{\mu}_{f}\left[0, x_{0}\right]+\widetilde{\mu}_{f}\left[x_{0}, x_{0}+\delta\right]\right) \\
& \geq \widetilde{F}_{f}\left(x_{0}\right)+\left|f\left(x_{0}^{+}\right)-f\left(x_{0}\right)\right|^{2}>\widetilde{F}_{f}\left(x_{0}\right) .
\end{aligned}
$$

Hence $x_{0} \in D_{\widetilde{F}_{f}}$.

(b) If $f$ is continuous at $x$, then by (a) we have that $\widetilde{F}_{f}(x)=F_{f}(x)$ or $\mu_{f}[0, x]=$ $\widetilde{\mu}_{f}[0, x]$. Again by (a) we have that $F_{f}$ is continuous at $x$ and so $\mu_{f}(\{x\})=0$. Hence $\mu_{f}[x, 1]=\mu_{f}[0,1]-\mu_{f}[0, x)=\mu_{f}[0,1]-\mu_{f}[0, x]=\widetilde{\mu}_{f}[0,1]-\widetilde{\mu}_{f}[0, x]=\widetilde{\mu}_{f}[x, 1]$, where the last equality follows from Lemma 13.

(c) Indeed, using (b) and Lemma [13, $\mu_{f}[x, y]=\mu_{f}[0, y]-\mu_{f}[0, x)=\mu_{f}[0, y]-$ $\mu_{f}[0, x]=\widetilde{\mu}_{f}[0, y]-\widetilde{\mu}_{f}[0, x]=\widetilde{\mu}_{f}[x, y]$.

(d) Let $a<a_{n}<b_{n}<b$ such that $a_{n}, b_{n}$ are continuity points of $f, \lim _{n} a_{n}=a$ and $\lim _{n} b_{n}=b$. By Lemma 14, $\lim _{n} \widetilde{\mu}_{f}\left(a, a_{n}\right]=\lim _{n} \widetilde{\mu}_{f}\left[b_{n}, b\right)=0$ and by Lemma $13 \widetilde{\mu}_{f}(a, b)=\widetilde{\mu}_{f}\left(a, a_{n}\right]+\widetilde{\mu}_{f}\left[a_{n}, b_{n}\right]+\widetilde{\mu}_{f}\left[b_{n}, b\right)$. Hence $\widetilde{\mu}_{f}(a, b)=\lim _{n} \widetilde{\mu}_{f}\left[a_{n}, b_{n}\right]=$ $\lim _{n} \mu_{f}\left[a_{n}, b_{n}\right]=\mu_{f}(a, b)$.

In order to proceed, we need the following notation. For every $f \in V_{2}$ and $x_{0} \in[0,1]$, let

$$
\tau_{f}\left(x_{0}\right)=\max \left\{\left|f\left(x_{0}^{+}\right)-f\left(x_{0}^{-}\right)\right|^{2},\left|f\left(x_{0}^{+}\right)-f\left(x_{0}\right)\right|^{2}+\left|f\left(x_{0}^{-}\right)-f\left(x_{0}\right)\right|^{2}\right\},
$$

where $f\left(0^{-}\right)=f(0)$ and $f\left(1^{+}\right)=f(1)$. Moreover, for every $\delta>0$, let

$$
\tau_{f, \delta}\left(x_{0}\right)=\sup \left\{\left|f(y)-f\left(x_{0}\right)\right|^{2}+\left|f\left(x_{0}\right)-f(z)\right|^{2},|f(y)-f(z)|^{2}\right\},
$$

where the $\max$ is taken for all $0 \leq y \leq x_{0} \leq z \leq 1$ with $|y-z| \leq \delta$. Clearly $\lim _{\delta \rightarrow 0} \tau_{f, \delta}\left(x_{0}\right)=\tau_{f}\left(x_{0}\right)$ and $f$ is continuous at $x_{0}$ if and only if $\tau_{f}\left(x_{0}\right)=0$. 
The quantity $\tau_{f}\left(x_{0}\right)$ is defined (with different notation) in [19], p.1464. An equivalent definition was introduced earlier by L.C. Young [23] in order to characterise the class $\mathcal{W}_{2}^{*}$. The proof of the next proposition uses similar arguments to those in [19].

Proposition 16. For every $f \in V_{2}$ and $x_{0} \in[0,1], \mu_{f}\left(\left\{x_{0}\right\}\right)=\tau_{f}\left(x_{0}\right)$. Therefore $\mu_{f}^{d}[0,1]=\sum_{x \in D_{f}} \tau_{f}(x)$, where $\mu_{f}^{d}$ denotes the discrete part of $\mu_{f}$.

Remark 2. Under the current terminology, it follows that $\mathcal{W}_{2}^{*}=\left\{f \in V_{2}: \mu_{f}=\mu_{f}^{d}\right\}$.

Lemma 17. Let $f_{1}, f_{2} \in V_{2}$ and $\tau$ be a positive Borel measure on $[0,1]$ such that $\mu_{f_{1}} \perp \tau$ and $\tau \leq \mu_{f_{2}}$. Then $\tau \leq \mu_{f_{2}-f_{1}}$.

Proof. Let $F=f_{1}-f_{2}$. It suffices to show that $\mu_{F}(V) \geq \tau(V)$, for every open subset $V$ of $[0,1]$. So fix an open subset $V$ of $[0,1]$ and let $\varepsilon>0$. Since $\mu_{f_{1}} \perp \tau$, there is $V_{\varepsilon} \subseteq V$ such that $V_{\varepsilon}=\bigcup_{i=1}^{k} I_{i}$, where $\left(I_{i}\right)_{i=1}^{k}$ is a finite family of pairwise disjoint open intervals of $[0,1], \mu_{f_{1}}\left(V_{\varepsilon}\right)<\varepsilon$ and $\tau\left(V_{\varepsilon}\right)>\tau(V)-\varepsilon$. Let $\delta>0$ be such that $\left|\widetilde{\mu}_{f_{1}, \delta}\left(V_{\varepsilon}\right)-\mu_{f_{1}}\left(V_{\varepsilon}\right)\right|<\varepsilon,\left|\widetilde{\mu}_{f_{2}, \delta}\left(V_{\varepsilon}\right)-\mu_{f_{2}}\left(V_{\varepsilon}\right)\right|<\varepsilon$ and $\left|\widetilde{\mu}_{F, \delta}\left(V_{\varepsilon}\right)-\mu_{F}\left(V_{\varepsilon}\right)\right|<\varepsilon$. Also for all $0 \leq i \leq k$, let $\mathcal{P}_{i} \subseteq I_{i}$ with $\left|\mathcal{P}_{i}\right|<\delta$ and $\left|\sum_{i=1}^{k} v_{2}^{2}\left(f_{2}, \mathcal{P}_{i}\right)-\mu_{f_{2}}\left(V_{\varepsilon}\right)\right|<\varepsilon$. Hence $\sum_{i=1}^{k} v_{2}^{2}\left(f_{1}, \mathcal{P}_{i}\right)<2 \varepsilon$ and

$$
\begin{aligned}
\mu_{F}(V) \geq \mu_{F}\left(V_{\varepsilon}\right) & >\widetilde{\mu}_{F, \delta}\left(V_{\varepsilon}\right)-\varepsilon \geq \sum_{i=1}^{k} v_{2}^{2}\left(F, \mathcal{P}_{i}\right)-\varepsilon \\
& \geq \sum_{i=1}^{k}\left[v_{2}\left(f_{2}, \mathcal{P}_{i}\right)-v_{2}\left(f_{1}, \mathcal{P}_{i}\right)\right]^{2}-\varepsilon \\
& \geq \mu_{f_{2}}\left(V_{\varepsilon}\right)-2\left(\sum_{i=1}^{k} v_{2}^{2}\left(f_{2}, \mathcal{P}_{i}\right)\right)^{1 / 2}\left(\sum_{i=1}^{k} v_{2}^{2}\left(f_{1}, \mathcal{P}_{i}\right)\right)^{1 / 2}-2 \varepsilon \\
& \geq \mu_{f_{2}}\left(V_{\varepsilon}\right)-2\left\|f_{2}\right\|_{V_{2}} \sqrt{2 \varepsilon}-2 \varepsilon \geq \tau\left(V_{\varepsilon}\right)-2\left\|f_{2}\right\|_{V_{2}} \sqrt{2 \varepsilon}-2 \varepsilon \\
& \geq \tau(V)-2\left\|f_{2}\right\|_{V_{2}} \sqrt{2 \varepsilon}-3 \varepsilon .
\end{aligned}
$$

Hence, letting $\varepsilon \rightarrow 0$, we get that $\mu_{F}(V) \geq \tau(V)$ and the proof is complete.

3.2. The correspondence between the functions of $V_{2}$ and the measures on the unit interval. By $\mathcal{M}[0,1]$ we denote the space of all Borel measures on $[0,1]$ endowed by the norm $\|\mu\|=\sup \{|\mu(B)|: B$ is a Borel subset of $[0,1]\}$. The positive cone of $\mathcal{M}[0,1]$ will be denoted by $\mathcal{M}^{+}[0,1]$. Recall that for every $\mu \in \mathcal{M}^{+}[0,1],\|\mu\|=\mu[0,1]$. In this subsection we study the properties of the function $\Phi: V_{2} \rightarrow \mathcal{M}[0,1]$, defined by $\Phi(f)=\mu_{f}$, for all $f \in V_{2}$. We start with the following easily established proposition.

Proposition 18. The following hold.

(i) For every $f_{1}, f_{2} \in V_{2}, \mu_{f_{1}+f_{2}} \leq 2 \mu_{f_{1}}+2 \mu_{f_{2}}$.

(ii) For every $f \in V_{2}$ and $\lambda \in \mathbb{R}, \mu_{(\lambda f)}=\lambda^{2} \mu_{f}$.

(iii) For every $f \in V_{2}$ and every $g \in V_{2}^{0}, \mu_{f+g}=\mu_{f}$.

(iv) The map $\Phi: V_{2} \rightarrow \mathcal{M}[0,1]$, defined by $\Phi(f)=\mu_{f}$, is locally Lipschitz. More precisely,

$$
\left\|\mu_{f_{1}}-\mu_{f_{2}}\right\| \leq\left(\left\|f_{1}\right\|_{V_{2}}+\left\|f_{2}\right\|_{V_{2}}\right)\left\|f_{1}-f_{2}\right\|_{V_{2}}
$$


Remark 3. One could not expect that $\Phi$ is a linear map as its range is a subset of the positive cone $\mathcal{M}^{+}[0,1]$ of $\mathcal{M}[0,1]$ (next we shall show that $\Phi$ is actually onto $\left.\mathcal{M}^{+}[0,1]\right)$. However there are special cases where the additivity of the function $\Phi$ is established. For example it can be shown that for every pair $f_{1}, f_{2} \in V_{2}$ with $\mu_{f_{1}} \perp \mu_{f_{2}}$ we have that $\mu_{f_{1}+f_{2}}=\mu_{f_{1}}+\mu_{f_{2}}$. Finally the map $\Phi$ is not $\mathrm{w}^{*}$-w* continuous. For example let $f \in V_{2}$ such that $f=\sum_{n} g_{n}$, where $\left(g_{n}\right)_{n}$ is a sequence in $V_{2}^{0}$. Then setting $f_{n}=\sum_{k \geq n} g_{k}$, we have that $\left(f_{n}\right)_{n}$ pointwise converges to 0 ; however, by (iii) of Proposition 18, $\mu_{f_{n}}=\mu_{f}$, for all $n \in \mathbb{N}$.

Lemma 19. Let $\mu$ be a finite positive discrete measure on $[0,1]$. Then there is $h \in V_{2}^{d}$ such that $\mu_{h}=\mu$.

Proof. Let $S=\left\{t_{n}\right\}_{n}$ be an enumeration of the support of $\mu$. Then $\mu^{d}=\sum_{n} \lambda_{n} \delta_{t_{n}}$, where $\lambda_{n}=\mu^{d}\left(\left\{t_{n}\right\}\right)$. We define $h=\sum_{n} \sqrt{\lambda_{n}} \chi_{t_{n}}$ and let $h_{n}=\sum_{k=1}^{n} \sqrt{\lambda_{k}} \chi_{t_{k}}$. Then $h \in V_{2}^{d},\left(h_{n}\right)_{n}\|\cdot\|_{V_{2}}$-converges to $h$ and so the $\left(\mu_{h_{n}}\right)_{n}$-norm converges to $\mu_{h}$ in $\mathcal{M}[0,1]$. Since $\mu_{h_{n}}=\sum_{k=1}^{n} \lambda_{k} \delta_{t_{k}}, \mu_{h}=\sum_{n} \lambda_{n} \delta_{t_{n}}=\mu$.

Theorem 20. For every finite positive Borel measure $\mu$ on $[0,1]$ there is $f \in V_{2}$ such that $\mu=\mu_{f}$.

Proof. Since $\mu=\mu^{c}+\mu^{d}$, where $\mu^{c}$ is the continuous and $\mu^{d}$ is the discrete part of $\mu$, by Lemma [19] it suffices to find $f \in V_{2} \cap C[0,1]$ such that $\mu^{c}=\mu_{f}$ (it is then easy to see that $\mu_{f+h}=\mu$, where $h \in V_{2}^{d}$ satisfies that $\mu_{h}=\mu^{d}$ ). Hence we suppose for the sequel that $\mu$ is continuous.

For an interval $I=[a, b]$ in $[0,1]$, let $F_{I}: I \rightarrow \mathbb{R}$ be defined by $F_{I}(x)=\mu[a, x]$, for all $x \in I$. Then $F_{I}$ is continuous, $F_{I}(a)=0$ and $F_{I}(b)=\mu(I)$. Hence we may choose $\xi_{I} \in(a, b)$ such that $F_{I}\left(\xi_{I}\right)=\mu\left[a, \xi_{I}\right]=\mu(I) / 2$. Now consider the function $G_{I}: I \rightarrow \mathbb{R}$ defined by $G_{I}(x)=F_{I}(x)$ if $a \leq x \leq \xi_{I}$ and by $G_{I}(x)=\mu(I)-F_{I}(x)$ if $\xi_{I} \leq x \leq b$. Clearly $\left\|G_{I}\right\|_{\infty}=\mu(I) / 2$.

Claim 1. For every interval $I$ of $[0,1]$, let $H_{I}=\sqrt{G_{I}}$.

(i) For every $x<y \in I,\left|H_{I}(y)-H_{I}(x)\right|^{2} \leq \mu(x, y]$.

(ii) For all intervals $I_{1}, I_{2}$ in $[0,1]$ such that $\max I_{1} \leq \min I_{2}$ and for all $x_{1} \in I_{1}$, $x_{2} \in I_{2},\left|H_{I_{2}}\left(x_{2}\right)-H_{I_{1}}\left(x_{1}\right)\right|^{2} \leq \mu\left(x_{1}, x_{2}\right]$.

Proof. (i) Notice that for $\alpha, \beta>0,|\alpha-\beta|^{2} \leq\left|\alpha^{2}-\beta^{2}\right|$. Hence $\left|H_{I}(y)-H_{I}(x)\right|^{2} \leq$ $\left|G_{I}(y)-G_{I}(x)\right|$ and so it suffices to show that $\left|G_{I}(y)-G_{I}(x)\right| \leq \mu(x, y]$. By the definition of $G_{I}$, we immediately get that for $x<y \leq \xi_{I}$ or for $\xi_{I} \leq x<$ $y,\left|G_{I}(y)-G_{I}(x)\right|=\mu(x, y]$. In the case $x<\xi_{I}<y$, we may assume that $G_{I}(x)<G_{I}(y)$ (the other case is similarly treated). Then there is $x<z<\xi_{I}$ with $G_{I}(z)=G_{I}(y)$ and so $\left|G_{I}(y)-G_{I}(x)\right|=\left|G_{I}(z)-G_{I}(x)\right|=\mu(x, z]<\mu(x, y]$.

(ii) As above it suffices to show that $\left|G_{I_{2}}\left(x_{2}\right)-G_{I_{1}}\left(x_{1}\right)\right| \leq \mu\left(x_{1}, x_{2}\right]$. Let $b_{1}$ be the right end of $I_{1}$ and $a_{2}$ be the left end of $I_{2}$. Then $G_{I_{1}}\left(b_{1}\right)=G_{I_{2}}\left(a_{2}\right)=0$ and by the proof of part (i), $\left|G_{I_{2}}\left(x_{2}\right)-G_{I_{1}}\left(x_{1}\right)\right|=\left|G_{I_{2}}\left(x_{2}\right)-G_{I_{2}}\left(a_{2}\right)-G_{I_{1}}\left(x_{1}\right)+G_{I_{1}}\left(b_{1}\right)\right| \leq$ $\left|G_{I_{2}}\left(x_{2}\right)-G_{I_{2}}\left(a_{2}\right)\right|+\left|G_{I_{1}}\left(b_{1}\right)-G_{I_{1}}\left(x_{1}\right)\right| \leq \mu\left(a_{2}, x_{2}\right]+\mu\left(x_{1}, b_{1}\right] \leq \mu\left(x_{1}, x_{2}\right]$.

Claim 2. Let $H_{n}=\sum_{i=1}^{2^{n+1}} H_{I_{i}^{n}}$, where $I_{i}^{n}=\left[\frac{i-1}{2^{n}}, \frac{i+1}{2^{n}}\right], n \geq 0,1 \leq i \leq 2^{n}$.

(i) $H_{n} \in V_{2}^{0}$ and $\left\|H_{n}\right\|_{\infty}=\sqrt{2^{-(n+1)} \mu[0,1]}$.

(ii) For all $\mathcal{I} \in \mathcal{A}, v_{2}^{2}\left(H_{n}, \mathcal{I}\right) \leq \mu(\bigcup \mathcal{I})$. Therefore $\left\|H_{n}\right\|_{V_{2}^{0}} \leq \sqrt{\mu[0,1]}$.

(iii) Let $\mathcal{P}_{n}=\left\{i / 2^{n}\right\}_{i=0}^{2^{n}} \cup\left\{\xi_{I_{i}^{n}}\right\}_{i=0}^{2^{n}}$. Then $v_{2}^{2}\left(H_{n}, \mathcal{P}_{n} \cap[0, t]\right)=\mu[0, t]$, for all $t \in \mathcal{P}_{n}$. 
Proof. This is straightforward by Claim 1 .

Claim 3. There exist a subsequence $\left(H_{n_{i}}\right)_{i}$ of $\left(H_{n}\right)_{n}$ and a strictly decreasing null sequence $\left(\delta_{i}\right)_{i=0}^{\infty}$, with $\delta_{0}=0$, satisfying the following.

(i) $\sum_{i}\left\|H_{n_{i}}\right\|_{\infty}<\infty$.

(ii) $\left(H_{n_{i}}\right)_{i}$ is $\left(\left(2^{-i}\right)_{i=1}^{\infty},\left(\delta_{i}\right)_{i=0}^{\infty}\right)$-biorthogonal.

(iii) For every $i \in \mathbb{N}, \delta_{i}<\left\|\mathcal{P}_{n_{i}}\right\|_{\min } \leq\left\|\mathcal{P}_{n_{i}}\right\|_{\max } \leq \delta_{i-1}$.

Proof. We inductively define a strictly increasing sequence $n_{1}<n_{2}<\ldots$ in $\mathbb{N}$ and a strictly decreasing sequence $1=\delta_{0}>\delta_{1}>\delta_{2}>\ldots>0$ such that for every $k \geq 1$, the following hold.

(1) For all $1 \leq i \leq k,\left\|H_{n_{i}}\right\|_{\infty}<2^{-i}$.

(2) The finite sequence $\left(H_{n_{i}}\right)_{i=1}^{k}$ is $\left(\left(2^{-i}\right)_{i=1}^{k},\left(\delta_{i}\right)_{i=0}^{k-1}\right)$-biorthogonal.

(3) For all $1 \leq i \leq k, \delta_{i}<\left\|\mathcal{P}_{n_{i}}\right\|_{\min } \leq\left\|\mathcal{P}_{n_{i}}\right\|_{\max } \leq \delta_{i-1}$.

(4) For every $\mathcal{I} \in \mathcal{A}$ with $\|\mathcal{I}\|_{\max } \leq \delta_{k}, \sum_{i=1}^{k} v_{2}\left(H_{i}, \mathcal{I}\right)<2^{-(k+1)}$.

The general inductive step of the construction goes as follows. Suppose that for some $k \geq 1$, we have chosen $\left(n_{i}\right)_{i \leq k}$ and $\left(\delta_{i}\right)_{i \leq k}$ satisfying the above. Applying Lemma 8 for $\varepsilon_{k+1}=2^{-(k+1)}$, we have that there exists $\varepsilon>0$ such that for every $H \in V_{2}^{0}$ with $\|H\|_{V_{2}^{0}}<\varepsilon$, the sequence $\left(H_{n_{1}}, \ldots, H_{n_{k}}, H\right)$ is $\left(\left(2^{-i}\right)_{i=1}^{k+1},\left(\delta_{i}\right)_{i=0}^{k}\right)$ biorthogonal. By Claim 2, we have that $\lim \left\|H_{n}\right\|_{\infty}=0$ and $\lim \left\|\mathcal{P}_{n}\right\|_{\max }=0$. Hence we may choose $n_{k+1}>n_{k}$ such that $\left\|H_{n_{k+1}}\right\|_{\infty}<\min \left\{2^{-k+1}, \varepsilon\right\}$ and $\left\|\mathcal{P}_{n_{k+1}}\right\|_{\max } \leq \delta_{k}$. Finally we choose $0<\delta_{k+1}<\left\|\mathcal{P}_{n_{k+1}}\right\|_{\text {min }}$ such that for every $\mathcal{I} \in \mathcal{A}$ with $\|\mathcal{I}\|_{\max } \leq \delta_{k+1}, \sum_{i=1}^{k+1} v_{2}\left(H_{n_{i}}, \mathcal{I}\right)<2^{-(k+2)}$ and the proof of the inductive step of the construction is complete.

Claim 4. Let $f=\sum_{i} H_{n_{i}}$. Then $f \in V_{2} \cap C[0,1]$ and $\mu_{f}=\mu$.

Proof. Since $\sum_{i}\left\|H_{n_{i}}\right\|_{\infty}<\infty$ and $H_{n_{i}}$ are continuous we have that $f \in C[0,1]$. By (ii) of Claim $2,\left(H_{n}\right)_{n}$ is a bounded (by $M=\sqrt{\mu[0,1]}$ ) sequence in $V_{2}^{0}$. Moreover by Claim 3, $\left(H_{n_{i}}\right)_{i}$ is biorthogonal and so by Lemma 11 and (ii) of Claim 2, we have that for every $\mathcal{I} \in \mathcal{A}$,

$$
v_{2}^{2}(f, \mathcal{I}) \leq \sum_{i} \mu\left(\bigcup \mathcal{I}^{(i)}\right)+2 \sqrt{\mu[0,1]}+1 \leq(\sqrt{\mu[0,1]}+1)^{2}
$$

and therefore $f \in V_{2}$. To prove that $\mu_{f}=\mu$, let $D=\bigcup_{i} D_{n_{i}}$ where for all $i \in \mathbb{N}$, $D_{n_{i}}=\left\{m 2^{-n_{i}}: 0 \leq m \leq 2^{n_{i}}\right\}$. Since $D$ is dense in $[0,1]$, it suffices to show that $\mu_{f}[0, t]=\mu[0, t]$, for all $t \in D$.

Fix $i_{0}$ and $0 \leq m_{0} \leq 2^{n_{i_{0}}}$ and let $t=m_{0} / 2^{n_{i_{0}}}$. By the definition of $\left(\mathcal{P}_{n}\right)_{n}$, we have that for all $j \geq i_{0}, t \in \mathcal{P}_{n_{i}}$, and so by (iii) of Claim 2 ,

$$
\mu[0, t]=v_{2}^{2}\left(H_{n_{j}}, \mathcal{P}_{n_{j}} \cap[0, t]\right) .
$$

Hence by (iii) of Claim 3 , for all $j \geq i_{0}$,

$$
v_{2}\left(f, \mathcal{P}_{n_{j}} \cap[0, t]\right)=v_{2}\left(H_{n_{j}}+\sum_{i \neq j} H_{n_{i}}, \mathcal{P}_{n_{j}} \cap[0, t]\right) \geq \mu[0, t]-2^{-j} ;
$$

hence since $\lim _{j}\left\|\mathcal{P}_{n_{j}}\right\|_{\max }=0, \mu_{f}[0, t] \geq \lim _{j} v_{2}^{2}\left(f, \mathcal{P}_{n_{j}} \cap[0, t]\right) \geq \mu[0, t]$. 
It remains to show that $\mu_{f}[0, t] \leq \mu[0, t]$. Since $\lim \delta_{k}=0$, we have that

$$
\mu_{f}[0, t]=\limsup _{k}\left\{v_{2}^{2}(f, \mathcal{P}): \mathcal{P} \subseteq[0, t],\|\mathcal{P}\|_{\max }<\delta_{k}\right\}
$$

Fix $k \geq 1$ and $\mathcal{P} \subseteq[0, t]$ such that $\|\mathcal{P}\|_{\max } \leq \delta_{k-1}$. Let $\mathcal{I}=\mathcal{I}_{\mathcal{P}}$ be the corresponding family of intervals with endpoints successive points of $\mathcal{P}$. Then $\mathcal{I}=\bigcup_{j \geq k} \mathcal{I}^{(j)}$, where $\mathcal{I}^{(j)}=\left\{I \in \mathcal{I}: \delta_{j}<|I| \leq \delta_{j-1}\right\}$, and

$$
v_{2}^{2}(f, \mathcal{P})=v_{2}^{2}(f, \mathcal{I})=v_{2}^{2}\left(f, \bigcup_{j \geq k} \mathcal{I}^{(j)}\right)=\sum_{j \geq k} v_{2}^{2}\left(f, \mathcal{I}^{(j)}\right)
$$

Moreover by (ii) of Claim 2,

$$
v_{2}\left(f, \mathcal{I}^{(j)}\right) \leq v_{2}\left(H_{n_{j}}+\sum_{i \neq j} H_{n_{i}}, \mathcal{I}^{(j)}\right) \leq v_{2}\left(H_{n_{j}}, \mathcal{I}^{(j)}\right)+2^{-j} \leq \sqrt{\mu\left(\bigcup \mathcal{I}^{(j)}\right)}+2^{-j} .
$$

Therefore $v_{2}^{2}\left(f, \mathcal{I}^{(j)}\right) \leq \mu\left(\bigcup \mathcal{I}^{(j)}\right)+(2 M+1) 2^{-j}$, where $M=\sqrt{\mu[0,1]}$, and so by (12) we obtain that

$$
\begin{aligned}
v_{2}^{2}(f, \mathcal{P}) & \leq \sum_{j \geq k} \mu\left(\bigcup \mathcal{I}^{(j)}\right)+(2 M+1) \sum_{j \geq k} 2^{-j} \\
& =\mu(\bigcup \mathcal{I})+(2 M+1) 2^{-(k-1)}=\mu[0, t]+(2 M+1) 2^{-(k-1)}
\end{aligned}
$$

Hence by (11) we get that $\mu_{f}[0, t] \leq \mu[0, t]$.

By Claim 4 the proof of the theorem is complete.

Remark 4. Let us present here a concrete example which illustrates the method of the proof of Theorem 20 . Let $\lambda$ be the Lebesgue measure on $[0,1]$ and let $\left(R_{n}\right)_{n}$ be defined by

$$
R_{n}(t)=2^{n / 2} \int_{0}^{t} r_{n}(x) d x
$$

where $\left(r_{n}\right)_{n}$ is the sequence of Rademacher functions. As in Claims 1 and 2 of Theorem 20, it can be shown that

(i) $v_{2}^{2}\left(R_{n}, \mathcal{I}\right) \leq \lambda(\bigcup \mathcal{I})$

(ii) for every $m \geq n$ and $1 \leq i \leq 2^{n}, v_{2}^{2}\left(R_{n}, \mathcal{P}_{m} \cap\left[0, \frac{i}{2^{n}}\right]\right)=\lambda\left[0, \frac{i}{2^{n}}\right]$,

where here $\mathcal{P}_{n}=\left\{\frac{i}{2^{n}}: 0 \leq i \leq 2^{n}\right\}$. Then as in Claim 3 we may show that there is a subsequence $\left(R_{n_{i}}\right)_{i}$ of $\left(R_{n}\right)_{n}$ such that the sum $f=\sum_{i} R_{n_{i}}$ satisfies that $\mu_{f}=\lambda$. We note that, as has been stated in [15], the above-defined sequence $\left(R_{n}\right)_{n}$ contains subsequences equivalent to the $c_{0}$-basis. In the sequel (Corollary [32) we shall provide a proof of this statement.

\subsection{On the points of non-differentiability of functions in $V_{2}$.}

Lemma 21. Let $f \in V_{2}$ and let $\left(\mathcal{P}_{n}\right)_{n}$ be a sequence of finite subsets of $[0,1]$ such that $\lim \left\|\mathcal{P}_{n}\right\|_{\max }=0$ and $\lim v_{2}^{2}\left(f, \mathcal{P}_{n}\right)=\mu_{f}[0,1]$. Then for every sequence $\left(\mathcal{I}_{n}\right)_{n}$ in $\mathcal{A}$ such that $\mathcal{I}_{n} \subseteq \mathcal{I}_{\mathcal{P}_{n}}$ and $\left(v_{2}^{2}\left(f, \mathcal{I}_{n}\right)\right)_{n}$ converges, we have that $\left(\mu_{f}\left(\bigcup \mathcal{I}_{n}\right)\right)_{n}$ also converges and $\lim v_{2}^{2}\left(f, \mathcal{I}_{n}\right)=\lim \mu_{f}\left(\bigcup \mathcal{I}_{n}\right)$.

Proof. Let $\alpha=\lim v_{2}^{2}\left(f, \mathcal{I}_{n}\right)$ and assume that $\left(\mu_{f}\left(\bigcup \mathcal{I}_{n}\right)\right)_{n}$ does not converge to $\alpha$. Then by passing to a subsequence, we may suppose that $\lim \mu_{f}\left(\bigcup \mathcal{I}_{n}\right)=\beta \neq \alpha$. 
Let $\mathcal{J}_{n}=\mathcal{I}_{\mathcal{P}_{n}} \backslash \mathcal{I}_{n}$. Then

$$
\lim v_{2}^{2}\left(f, \mathcal{J}_{n}\right)=\mu_{f}[0,1]-\alpha \text { and } \lim \mu_{f}\left(\bigcup \mathcal{J}_{n}\right)=\mu_{f}[0,1]-\beta .
$$

Since $\mathcal{I}_{n}$ and $\mathcal{J}_{n}$ consist of open intervals of $[0,1]$, we can choose $\mathcal{I}_{n}^{\prime} \preceq \mathcal{I}_{n}$ and $\mathcal{J}_{n}^{\prime} \preceq \mathcal{J}_{n}$ such that $\left|\mu_{f}\left(\bigcup \mathcal{I}_{n}\right)-v_{2}^{2}\left(f, \mathcal{I}_{n}^{\prime}\right)\right|<1 / n$ and $\left|\mu_{f}\left(\bigcup \mathcal{J}_{n}\right)-v_{2}^{2}\left(f, \mathcal{J}_{n}^{\prime}\right)\right|<1 / n$. Therefore we get that

$$
\lim v_{2}^{2}\left(f, \mathcal{I}_{n}^{\prime}\right)=\beta \text { and } \lim v_{2}^{2}\left(f, \mathcal{J}_{n}^{\prime}\right)=\mu_{f}[0,1]-\beta .
$$

Since $\mathcal{I}_{n}, \mathcal{J}_{n}^{\prime}$ are disjoint and $\lim \left\|\mathcal{I}_{n}\right\|_{\max }=\lim \left\|\mathcal{J}_{n}^{\prime}\right\|_{\max }=0$ we obtain that

$$
\mu_{f}[0,1] \geq \lim v_{2}^{2}\left(f, \mathcal{I}_{n} \cup \mathcal{J}_{n}^{\prime}\right)=\alpha+\left(\mu_{f}[0,1]-\beta\right),
$$

which implies that $\beta \geq \alpha$. Similarly,

$$
\mu_{f}[0,1] \geq \lim v_{2}^{2}\left(f, \mathcal{I}_{n}^{\prime} \cup \mathcal{J}_{n}\right)=\beta+\left(\mu_{f}[0,1]-\alpha\right),
$$

which gives that $\alpha \geq \beta$. Hence $\alpha=\beta$, which is a contradiction.

Theorem 22. Let $f \in V_{2}$. Then the set of all points $x \in[0,1]$ such that $f$ is differentiable at $x$ has $\mu_{f}$-measure zero.

Proof. Let $\mathcal{P}_{n}=\left\{0=t_{0}^{n}<\ldots<t_{k_{n}}^{n}=1\right\} \subseteq[0,1]$ such that $\lim \left\|\mathcal{P}_{n}\right\|_{\max }=0$ and $\lim v_{2}^{2}\left(f, \mathcal{P}_{n}\right)=\mu_{f}([0,1])$. It suffices to show that for every $C>0, \mu_{f}\left(A_{C}\right)=0$, where

$$
A_{C}=\left\{x \in[0,1]: \exists f^{\prime}(x) \text { and }\left|f^{\prime}(x)\right|<C\right\} .
$$

Fix $C>0$ and for every $k \in \mathbb{N}$ let $A_{C}^{k}$ be the set of all $x \in(0,1) \backslash \bigcup_{n} \mathcal{P}_{n}$ such that for every $y, z \in(x-1 / k, x+1 / k)$ with $0 \leq y<x<z \leq 1,\left|\frac{f(z)-f(y)}{z-y}\right|<C$.

Notice that

$$
A_{C} \subseteq\left(\bigcup_{n} \mathcal{P}_{n} \backslash D_{f}\right) \cup \bigcup_{k=1}^{\infty} A_{C}^{k} .
$$

By Proposition [16, we get that $\mu_{f}\left(\bigcup_{n} \mathcal{P}_{n} \backslash D_{f}\right)=0$, and therefore it remains to show that for every $k \in \mathbb{N}, \mu_{f}\left(A_{C}^{k}\right)=0$. To this end, fix $k \in \mathbb{N}$. Then for every $x \in A_{C}^{k}$ and $n \in \mathbb{N}$ there exists $0 \leq i \leq k_{n}-1$ such that $x \in\left(t_{i}^{n}, t_{i+1}^{n}\right)$. Since $\lim \left\|\mathcal{P}_{n}\right\|_{\max }=0$ there is $n_{0}$ such that for all $n \geq n_{0}, t_{i+1}^{n}-t_{i}^{n}<1 / k$. For $n \geq n_{0}$ let $F_{n}$ be the set of all $0 \leq i \leq k_{n}-1$ such that $A_{C}^{k} \cap\left(t_{i}^{n}, t_{i+1}^{n}\right) \neq \emptyset$ and let $\mathcal{I}_{n}=\left(\left(t_{i}^{n}, t_{i+1}^{n}\right)\right)_{i \in F_{n}}$. Then $A_{C}^{k} \subseteq \bigcup \mathcal{I}_{n}$ and therefore

$$
v_{2}^{2}\left(f, \mathcal{I}_{n}\right)=\sum_{i \in F_{n}}\left|f\left(t_{i+1}^{n}\right)-f\left(t_{i}^{n}\right)\right|^{2} \leq C^{2} \sum_{i \in F_{n}}\left|t_{i+1}^{n}-t_{i}^{n}\right|^{2} \leq C^{2} \max _{i \in F_{n}}\left|t_{i+1}^{n}-t_{i}^{n}\right| .
$$

Hence $\lim v_{2}^{2}\left(f, \mathcal{I}_{n}\right)=0$. By Lemma 21, $\lim \mu_{f}\left(\bigcup \mathcal{I}_{n}\right)=0$ and so $\mu_{f}\left(A_{C}^{k}\right)=0$.

Corollary 23. Let $f \in V_{2} \cap C[0,1]$. If the set of all points $x \in[0,1]$ such that $f$ is not differentiable at $x$ is countable, then $f \in V_{2}^{0}$. Moreover if $f \in\left(V_{2} \backslash V_{2}^{0}\right) \cap C[0,1]$, then the set of all non-differentiability points of $f$ contains a perfect set.

Proof. Let $B$ be the set of all $x \in[0,1]$ such that $f$ is not differentiable at $x$. By Theorem 22, we have that $\mu_{f}(B)=\mu_{f}[0,1]$. Also since $f \in C[0,1], \mu_{f}$ is 
continuous. Therefore if $B$ is countable, then $\mu_{f}[0,1]=0$ and so $f \in V_{2}^{0}$. In the case $f \in\left(V_{2} \backslash V_{2}^{0}\right) \cap C[0,1], \mu_{f}(B)>0$ and so $B$ contains a perfect set.

\section{Geometric properties of the measure}

In this section we are mainly concerned with connecting the norm of the measure $\mu_{f}$ with the distance of $f$ from $V_{2}^{0}$. This first requires some results from [2], included in the first subsection, related to the oscillation function $\widetilde{o s c} f$ defined by A. Kechris and A. Louveau [13] and further studied by H. P. Rosenthal in 21]. The second subsection contains the statement and the proof of the basic inequality and in the third subsection we use these geometric properties of the measure to obtain optimal approximations for the functions of $V_{2} \backslash V_{2}^{0}$.

4.1. The oscillation function. Recall that for a function $f: K \rightarrow \mathbb{R}$, where $K$ is a compact metric space, $\widetilde{o s c}_{K} f$ is defined as follows. For every $t \in V \subseteq K$ let $s(V, t)=\sup \{|f(x)-f(t)|: x \in V\}$. Then for each $t \in K$,

$$
\widetilde{o s c}_{K} f(t)=\inf \{s(V, t): V \text { open neighborhood of } t\} .
$$

It can be easily shown that for every sequence $\left(f_{n}\right)_{n}$ of continuous real-valued functions on $K$ pointwise converging to a function $f$ and every $\varepsilon>0$ there exists $n_{0} \in \mathbb{N}$ such that for every $n \geq n_{0},\left\|\widetilde{o s c_{K}} f\right\|_{\infty}-\varepsilon<\left\|f_{n}-f\right\|_{\infty}$.

The next lemma is included in the more general Lemma 1.2 in [2] and shows that, passing to convex blocks, the above inequality can be reversed.

Lemma 24. Let $\left(f_{n}\right)_{n}$ be a uniformly bounded sequence of continuous real-valued functions on a compact metric space $K$ pointwise converging to a function $f$. Then for every null sequence of positive reals $\left(\delta_{n}\right)_{n}$ there exists a convex block sequence $\left(g_{n}\right)_{n}$ of $\left(f_{n}\right)_{n}$ such that $\left\|g_{n}-f\right\|_{\infty}<\left\|\widetilde{o s c_{K}} f\right\|_{\infty}+\delta_{n}$.

Corollary 25. Let $E$ be a separable Banach space, $X$ be a subspace of $E$ and $K$ be a $w^{*}$-compact subset of $B_{E^{*}}$ which is 1-norming for $E^{* *}$ (that is, for all $\left.x^{* *} \in E^{* *},\left\|x^{* *}\right\|=\sup _{x^{*} \in K}\left|x^{* *}\left(x^{*}\right)\right|\right)$. Then for all $x^{* *} \in X^{* *}$ which are $w^{*}$ limits of sequences in $X$, we have that

$$
\operatorname{dist}\left(x^{* *}, X\right) \leq\left\|\widetilde{o s c}_{K} x^{* *}\right\|_{\infty} .
$$

In particular this holds for all $x^{* *} \in X^{* *}$ if $\ell_{1}$ is not embedded into $X$.

Proof. Let $K$ be a $\mathrm{w}^{*}$ subset of $B_{E^{*}}$ which is 1-norming for $X^{* *}$. Let $x^{* *} \in X^{* *}$ be the $\mathrm{w}^{*}$ - limit of a sequence $\left(x_{n}\right)_{n}$ in $X$. Denoting again by $x_{n}$ and $x^{* *}$ the restrictions of $x_{n}$ and $x^{* *}$ on $K$, we have that $\left(x_{n}\right)_{n}$ is a uniformly bounded sequence of continuous functions on the compact metric space $K$, pointwise convergent to $x^{* *}$. Therefore, by Lemma 24 there is a convex block sequence $\left(y_{n}\right)_{n}$ of $\left(x_{n}\right)_{n}$ such that for all $n \in \mathbb{N}$,

$$
\sup _{x^{*} \in K}\left|y_{n}\left(x^{*}\right)-x^{* *}\left(x^{*}\right)\right| \leq\left\|\widetilde{o s c_{K}} x^{* *}\right\|_{\infty}+1 / n .
$$

Since $K$ is 1-norming for $E^{* *}$, we have that the left side of the above inequality is the norm of $y_{n}-x^{* *}$. Hence

$$
\operatorname{dist}\left(x^{* *}, X\right) \leq\left\|y_{n}-x^{* *}\right\| \leq\left\|\widetilde{o s c}_{K} x^{* *}\right\|_{\infty}+1 / n,
$$

for all $n \in \mathbb{N}$ and the result follows. Finally if $\ell_{1}$ does not embed into $X$, then by Odell-Rosenthal's theorem [18, all $x^{* *} \in X^{* *}$ are $\mathrm{w}^{*}$-limits of sequences of $X$. 
Remark 5. Let $E, X$ and $K$ be as in Corollary 25. Then notice that for every $x^{* *} \in X^{* *}$ and every $x \in X,\left\|\widetilde{o s c}_{K} x^{* *}\right\|_{\infty}=\left\|\widetilde{o s c_{K}}\left(x^{* *}-x\right)\right\|_{\infty} \leq 2\left\|x^{* *}-x\right\|$ and so $\left\|\widetilde{o s c}_{K} x^{* *}\right\|_{\infty} \leq 2 \operatorname{dist}\left(x^{* *}, X\right)$. Hence by Corollary 25] we have that if $X$ does not contain $\ell_{1},\left\|\widetilde{\sigma s c_{K}} x^{* *}\right\|_{\infty}$ is an equivalent norm on the quotient space $X^{* *} / X$.

4.2. Connection of the measure $\mu_{f}$ with the distance of $f$ from $V_{2}^{0}$. Returning to $V_{2}$, we consider the following subset of $\left(V_{2}^{0}\right)^{*}$ introduced in 3 :

$$
\mathcal{K}=\left\{\sum_{i=1}^{\infty} \alpha_{i}\left(\delta_{s_{i}}-\delta_{t_{i}}\right):\left(\left(s_{i}, t_{i}\right)\right)_{i} \text { are disjoint intervals and } \sum_{i} \alpha_{i}^{2} \leq 1\right\}
$$

We recall ([3]) that $\mathcal{K}$ is a $\mathrm{w}^{*}$-compact subset of $B_{\left(V_{2}^{0}\right)^{*}}, 1$-norming $V_{2}$.

Theorem 26. Let $X$ be a subspace of $V_{2}^{0}$. Then for every $f \in X^{* *}$,

$$
\sqrt{\left\|\mu_{f}\right\|} \leq \operatorname{dist}\left(f, V_{2}^{0}\right) \leq \operatorname{dist}(f, X) \leq\left\|\widetilde{o s \mathcal{K}_{\mathcal{K}}} f\right\|_{\infty} \leq \sqrt{\left\|\mu_{f}\right\|}+2 \sqrt{\left\|\mu_{f}^{d}\right\|} .
$$

Proof. By Proposition [18, we have that for every $g \in V_{2}^{0}, \mu_{f+g}=\mu_{f}$. Hence for every $g \in V_{2}^{0},\left\|\mu_{f}\right\|=\left\|\mu_{f+g}\right\| \leq\|f+g\|_{V_{2}}^{2}$, which gives that $\sqrt{\left\|\mu_{f}\right\|} \leq \operatorname{dist}\left(f, V_{2}^{0}\right) \leq$ $\operatorname{dist}(f, X)$. Since $\mathcal{K}$ is a $\mathrm{w}^{*}$-compact subset of $\left(V_{2}^{0}\right)^{*}$ which is 1 -norming for $V_{2}$ and $\ell_{1}$ is not embedded into $V_{2}^{0}$, by Corollary 25, we have that $\operatorname{dist}(f, X) \leq\left\|\widetilde{o s c} \mathcal{K}_{\mathcal{K}} f\right\|_{\infty}$ and so it remains to prove that $\left\|\widetilde{O S c_{\mathcal{K}}} f\right\|_{\infty} \leq \sqrt{\left\|\mu_{f}\right\|}+2 \sqrt{\left\|\mu_{f}^{d}\right\|}$. To show this, let $x^{*} \in \mathcal{K}$ and let $\left\{x_{n}^{*}\right\}_{n}$ be a sequence in $\mathcal{K}, w^{*}$-converging to $x^{*}$ such that $\widetilde{o s c} \mathcal{K} f\left(x^{*}\right)=\lim _{n}\left|f\left(x_{n}^{*}\right)-f\left(x^{*}\right)\right|$ (clearly there exists such a sequence). Notice that for every $y^{*}=\sum_{i} \alpha_{i}\left(\delta_{s_{i}}-\delta_{t_{i}}\right) \in \mathcal{K}$ and every $f \in V_{2}, f\left(y^{*}\right)=\sum_{i} \alpha_{i}\left(f\left(s_{i}\right)-f\left(t_{i}\right)\right)$, where the series $\sum_{i} \alpha_{i}\left(f\left(s_{i}\right)-f\left(t_{i}\right)\right)$ is absolutely convergent. So we may reorder each $x_{n}^{*}$ and in this way we may assume that $x_{n}^{*}=\sum_{i=1}^{\infty} \alpha_{i}^{n}\left(\delta_{s_{i}^{n}}-\delta_{t_{i}^{n}}\right)$, where for every $i, t_{i}^{n}-s_{i}^{n} \geq t_{i+1}^{n}-s_{i+1}^{n}$. Moreover by passing to a subsequence we may also suppose that for each $i \in \mathbb{N}$, the sequences $\left\{s_{i}^{n}\right\}_{n},\left\{t_{i}^{n}\right\}_{n}$ are monotone and that $\alpha_{i}^{n} \rightarrow \alpha_{i}, s_{i}^{n} \rightarrow s_{i}$, and $t_{i}^{n} \rightarrow t_{i}$.

Therefore $x^{*}=\sum_{i=1}^{\infty} \alpha_{i}\left(\delta_{s_{i}}-\delta_{t_{i}}\right)$ with $t_{i}-s_{i} \geq t_{i+1}-s_{i+1}$ and so since $\left(\left(s_{i}, t_{i}\right)\right)_{i}$ consists of pairwise disjoint open intervals in $[0,1]$ of decreasing length, $t_{i}-s_{i} \leq 1 / i$. Also by the monotonicity of $\left(s_{i}^{n}\right)_{n},\left(t_{i}^{n}\right)_{n}$, there are $\varepsilon_{t_{i}}, \varepsilon_{s_{i}} \in\{0,+,-\}$, such that $\lim _{n} f\left(t_{i}^{n}\right)=f\left(t_{i}^{\varepsilon_{t_{i}}}\right)$ and $\lim _{n} f\left(s_{i}^{n}\right)=f\left(s_{i}^{\varepsilon_{s_{i}}}\right)$, where $t_{i}^{0}=t_{i}$ and $s_{i}^{0}=s_{i}$.

Let $\varepsilon>0$. We choose $i_{1} \in \mathbb{N}$ such that $\mu_{f}[0,1] \leq \widetilde{\mu}_{f, 1 / i_{1}}[0,1]<\mu_{f}[0,1]+\varepsilon^{2}$ and $\sum_{i=i_{1}+1}^{\infty}\left|\alpha_{i}\right|^{2}<\varepsilon^{2} /\|f\|_{V_{2}}^{2}$. We set

$$
x_{1}^{*}=\sum_{i=1}^{i_{1}} \alpha_{i}\left(\delta_{s_{i}}-\delta_{t_{i}}\right) \quad \text { and } \quad x_{2}^{*}=\sum_{i=i_{1}+1}^{\infty} \alpha_{i}\left(\delta_{s_{i}}-\delta_{t_{i}}\right)
$$

and for each $n \in \mathbb{N}$, let

$$
x_{n, 1}^{*}=\sum_{i=1}^{i_{1}} \alpha_{i}^{n}\left(\delta_{s_{i}^{n}}-\delta_{t_{i}^{n}}\right) \quad \text { and } \quad x_{n, 2}^{*}=\sum_{i=i_{1}+1}^{\infty} \alpha_{i}^{n}\left(\delta_{s_{i}^{n}}-\delta_{t_{i}^{n}}\right) .
$$


Then by the Cauchy-Schwarz inequality we get that $\left|f\left(x_{2}^{*}\right)\right|^{2} \leq \varepsilon^{2}$ and $\left|f\left(x_{n, 2}^{*}\right)\right|^{2} \leq$ $\mu_{f}[0,1]+\varepsilon^{2}$, for all $n \in \mathbb{N}$. Therefore

$$
\begin{aligned}
& \widetilde{o s} \mathcal{K}_{\mathcal{K}} f\left(x^{*}\right)=\lim _{n}\left|f\left(x_{n}^{*}\right)-f\left(x^{*}\right)\right| \leq\left|\lim _{n} f\left(x_{n, 1}^{*}\right)-f\left(x_{1}^{*}\right)\right|+\varlimsup_{n}\left|f\left(x_{n, 2}^{*}\right)-f\left(x_{2}^{*}\right)\right| \\
& \leq\left|\sum_{i=1}^{i_{1}} \alpha_{i}\left(f\left(s_{i}^{\varepsilon_{s_{i}}}\right)-f\left(s_{i}\right)\right)\right|+\left|\sum_{i=1}^{i_{1}} \alpha_{i}\left(f\left(t_{i}^{\varepsilon_{t_{i}}}\right)-f\left(t_{i}\right)\right)\right|+\varlimsup_{n}\left|f\left(x_{n, 2}^{*}\right)\right|+\left|f\left(x_{2}^{*}\right)\right| \\
& \leq\left(\sum_{i=1}^{i_{1}}\left|f\left(s_{i}^{\varepsilon_{s_{i}}}\right)-f\left(s_{i}\right)\right|^{2}\right)^{1 / 2}+\left(\sum_{i=1}^{i_{1}}\left|f\left(t_{i}^{\varepsilon_{t_{i}}}\right)-f\left(t_{i}\right)\right|^{2}\right)^{1 / 2}+\left(\sqrt{\mu_{f}[0,1]}+\varepsilon\right)+\varepsilon \\
& \leq\left(\sum_{i=1}^{i_{1}} \mu_{f}\left(\left\{s_{i}\right\}\right)\right)^{1 / 2}+\left(\sum_{i=1}^{i_{1}} \mu_{f}\left(\left\{t_{i}\right\}\right)\right)^{1 / 2}+\sqrt{\mu_{f}[0,1]}+2 \varepsilon .
\end{aligned}
$$

Hence for every $\varepsilon>0,\left\|\widetilde{o s} \mathcal{K}_{\mathcal{K}} f\right\|_{\infty} \leq \sqrt{\left\|\mu_{f}\right\|}+2 \sqrt{\left\|\mu_{f}^{d}\right\|}+2 \varepsilon$ and the conclusion follows.

Since $\mu_{f}^{d}=0$ if $f$ is continuous, we easily get the following.

Corollary 27. For every subspace $X$ of $V_{2}^{0}$ and every $f \in X^{* *} \cap C[0,1]$,

$$
\operatorname{dist}\left(f, V_{2}^{0}\right)=\operatorname{dist}(f, X)=\left\|\widetilde{o s} c_{\mathcal{K}} f\right\|_{\infty}=\sqrt{\left\|\mu_{f}\right\|} .
$$

Remark 6. Let us note that there exist non-continuous functions $f \in V_{2}$ satisfying the proper inequalities $\sqrt{\left\|\mu_{f}\right\|}<\operatorname{dist}\left(f, V_{2}^{0}\right)<\left\|\widetilde{o s c_{\mathcal{K}}} f\right\|_{\infty}$. For example, it can be easily shown that for $0<s<t<1$ and $f=\chi_{[s, t]}+2 \chi_{(t, 1]}$, we have that $\left\|\widetilde{o s c} \mathcal{K}_{\mathcal{K}} f\right\|_{\infty}=2, \mu_{f}$ is the sum of the Dirac measures on $s$ and $t$ (so $\sqrt{\mu_{f}}=\sqrt{\mu_{f}^{d}}=$ $\sqrt{2})$ and $\sqrt{2}<\operatorname{dist}\left(f, V_{2}^{0}\right)<2$.

\subsection{Optimal approximation of functions of $V_{2} \backslash V_{2}^{0}$.}

Lemma 28. Let $f \in V_{2} \backslash V_{2}^{0}$ and let $\left(f_{n}\right)_{n}$ be a bounded sequence in $V_{2}^{0}$ pointwise convergent to $f$. Then for every sequence $\left(\varepsilon_{n}\right)_{n}$ of positive real numbers there exists a convex block sequence $\left(h_{n}\right)_{n}$ of $\left(f_{n}\right)_{n}$ satisfying the following properties:

(i) $\left\|h_{n}-f\right\|_{\infty} \leq\|\widetilde{o s c}[0,1] f\|_{\infty}+\varepsilon_{n}$;

(ii) for every $\mathcal{I} \in \mathcal{F}\left([0,1] \backslash D_{f}\right), v_{2}^{2}\left(h_{n}-f, \mathcal{I}\right) \leq \mu_{f}(\bigcup \mathcal{I})+8\|f\|_{V_{2}} \sqrt{\left\|\mu_{f}^{d}\right\|}+\varepsilon_{n}$.

Proof. We may assume that $\varepsilon_{n}<1$. Also let $\left(\delta_{n}\right)_{n}$ be a decreasing sequence of positive real numbers such that $\delta_{n} \leq\left(1+6\|f\|_{V_{2}}\right)^{-1} \varepsilon_{n}$, for all $n$. By our assumptions we have that $\left(f_{n}\right)_{n}$ is a uniformly bounded sequence in $C[0,1]$ pointwise convergent to $f$. Hence by Lemma 24 (for $K=[0,1]$ ), there exists a convex block sequence $\left(g_{n}\right)_{n}$ of $\left(f_{n}\right)_{n}$ such that

$$
\left\|g_{n}-f\right\|_{\infty} \leq\|\widetilde{O S C}[0,1] f\|_{\infty}+\delta_{n} .
$$

Next we view each $g_{n}$ as well as $f$ as functions acting on $\mathcal{K}$. Since $\mathcal{K}$ is a subset of the unit ball of $B_{\left(V_{2}^{0}\right)^{*}}$, 1-norming $V_{2}$, we conclude that the sequence $\left(g_{n}\right)_{n}$ is uniformly bounded in $C\left(\mathcal{K}, \mathrm{w}^{*}\right)$ pointwise convergent to $f$ and also $\left\|g_{n}-f\right\|_{\mathcal{K}}=\left\|g_{n}-f\right\|_{V_{2}}$. Hence again by Lemma 24 (for $K=\mathcal{K}$ ) and Theorem 26, there exists a convex block sequence $\left(h_{n}\right)_{n}$ of $\left(g_{n}\right)_{n}$ such that

$$
\left\|h_{n}-f\right\|_{V_{2}}=\left\|h_{n}-f\right\|_{\mathcal{K}} \leq\left\|\widetilde{o s} \mathcal{K}_{\mathcal{K}} f\right\|_{\infty}+\delta_{n} \leq \sqrt{\left\|\mu_{f}\right\|}+2 \sqrt{\left\|\mu_{f}^{d}\right\|}+\delta_{n} .
$$


Let $\left(F_{n}\right)_{n}$ be a block sequence of finite subsets of $\mathbb{N}$ and $\left(\lambda_{i}\right)_{i}$ be a sequence of positive real numbers of $[0,1]$ such that for each $n \in \mathbb{N}, \sum_{i \in F_{n}} \lambda_{i}=1$ and $h_{n}=$ $\sum_{i \in F_{n}} \lambda_{i} g_{i}$. Then considering $h_{n}$ and $f$ as functions on $[0,1]$, by (15) we obtain that

$$
\begin{aligned}
\left\|h_{n}-f\right\|_{\infty} & \leq \sum_{i \in F_{n}} \lambda_{i}\left\|g_{i}-f\right\|_{\infty} \leq \sum_{i \in F_{n}} \lambda_{i}\left\|\widetilde{o s c}_{[0,1]} f\right\|_{\infty}+\sum_{i \in F_{n}} \lambda_{i} \delta_{i} \\
& \leq\left\|\widetilde{o s c} C_{[0,1]} f\right\|_{\infty}+\delta_{n},
\end{aligned}
$$

as $n \leq \min F_{n}$ and $\left(\delta_{n}\right)_{n}$ is decreasing. Moreover, using that $\left\|\mu_{f}^{d}\right\|=\sqrt{\left\|\mu_{f}^{d}\right\|} \sqrt{\left\|\mu_{f}^{d}\right\|}$ $\leq\|f\|_{V_{2}} \sqrt{\left\|\mu_{f}^{d}\right\|}$ and taking squares in (16) we easily obtain that

$$
\left\|h_{n}-f\right\|_{V_{2}}^{2} \leq\left\|\mu_{f}\right\|+8\|f\|_{V_{2}} \sqrt{\left\|\mu_{f}^{d}\right\|}+\varepsilon_{n} .
$$

Let $\mathcal{I} \in \mathcal{F}\left([0,1] \backslash D_{f}\right)$. Then $[0,1] \backslash \bigcup \mathcal{I}=\bigcup_{i=1}^{m} I_{i}^{\prime}$, where each $I_{i}^{\prime}$ is a non-trivial open interval in $[0,1]$. By Proposition [15, $\mu_{f}\left(I_{i}^{\prime}\right)=\widetilde{\mu}_{f}\left(I_{i}^{\prime}\right)$ and so for each $1 \leq i \leq m$, we can choose a sequence $\left(\mathcal{P}_{k}^{i}\right)_{k}$ of finite subsets of $I_{i}^{\prime}$, such that $\lim _{k}\left\|\mathcal{P}_{k}^{i}\right\|_{\max }=0$ and $\lim _{k} v_{2}^{2}\left(f, \mathcal{P}_{k}^{i}\right)=\mu_{f}\left(I_{i}^{\prime}\right)$. Since $h_{n} \in V_{2}^{0}$, for all $1 \leq i \leq m$,

$$
\lim _{k} v_{2}^{2}\left(h_{n}-f, \mathcal{P}_{k}^{i}\right)=\lim _{k} v_{2}^{2}\left(f, \mathcal{P}_{k}^{i}\right)=\mu_{f}\left(I_{i}^{\prime}\right) .
$$

Also setting $\mathcal{I}^{\prime}=\left(I_{i}^{\prime}\right)_{i=1}^{m},\left\|\mu_{f}\right\|=\mu_{f}[0,1]=\mu_{f}(\bigcup \mathcal{I})+\mu_{f}\left(\bigcup \mathcal{I}^{\prime}\right)$. Hence (18) gives that

$$
v_{2}^{2}\left(h_{n}-f, \mathcal{I}\right)+\sum_{i=1}^{m} v_{2}^{2}\left(h_{n}-f, \mathcal{P}_{k}^{i}\right) \leq \mu_{f}(\bigcup \mathcal{I})+\mu_{f}\left(\bigcup \mathcal{I}^{\prime}\right)+8\|f\|_{V_{2}} \sqrt{\left\|\mu_{f}^{d}\right\|}+\varepsilon_{n} .
$$

Letting $k \rightarrow \infty$ and using (19), part (ii) of the lemma follows.

Proposition 29. Let $X$ be a subspace of $V_{2}^{0}, f \in X^{* *} \backslash X$ and $\left(f_{n}\right)_{n}$ be a bounded sequence in $X$ pointwise convergent to $f$. Then for every $0<\delta<\operatorname{dist}(f, X)$ and for every sequence $\left(\varepsilon_{n}\right)_{n}$ of positive real numbers there exists a convex block sequence $\left(h_{n}\right)_{n}$ of $\left(f_{n}\right)_{n}$ such that for all $n<m$ the following properties are satisfied:

(i) $\delta<\left\|h_{m}-h_{n}\right\|_{V_{2}} \leq 2 M$, where $M=\sup _{n}\left\|f_{n}\right\|_{V_{2}}$;

(ii) $\left\|h_{m}-h_{n}\right\|_{\infty} \leq 2\left\|\widetilde{o s c} c_{[0,1]} f\right\|_{\infty}+\varepsilon_{n} \leq 4\|f\|_{\infty}+\varepsilon_{n}$;

(iii) for every $\mathcal{I} \in \mathcal{A}, v_{2}^{2}\left(h_{m}-h_{n}, \mathcal{I}\right) \leq 4 \mu_{f}(\bigcup \mathcal{I})+32\|f\|_{V_{2}} \sqrt{\left\|\mu_{f}^{d}\right\|}+\varepsilon_{n}$.

Moreover given $k \in \mathbb{N}$ and an open subset $V$ of $[0,1]$ with $\mu_{f}(V)>\theta>0$ there exist $\mathcal{J} \in \mathcal{A}$ with $\bigcup \mathcal{J} \subseteq V$ and $l>k$ such that

(iv) $v_{2}^{2}\left(h_{l}-h_{k}, \mathcal{J}\right)>\theta$.

Proof. Let $\left(\varepsilon_{n}^{\prime}\right)$ be a decreasing sequence of positive real numbers with $\varepsilon_{n}^{\prime}<4 \varepsilon_{n}$. Let $\left(h_{n}\right)_{n}$ be the convex block sequence of $\left(f_{n}\right)_{n}$ resulting from Lemma 28, Since $\left(h_{n}\right)_{n}$ is $\mathrm{w}^{*}$ - convergent to $f$, for every $n \in \mathbb{N}$ there are finitely many $m>n$ such that $\left\|h_{m}-h_{n}\right\|_{V_{2}} \leq \delta$ (otherwise, $\left\|f-h_{n}\right\|_{V_{2}} \leq \delta<\operatorname{dist}(f, X)$, which is impossible). Therefore by passing to a subsequence we may assume that for all $n<m, \delta<\left\|h_{m}-h_{n}\right\|_{V_{2}}$. Also since $\left(h_{n}\right)_{n}$ is a convex block sequence of $\left(f_{n}\right)_{n}$, $\left\|h_{n}\right\|_{V_{2}} \leq M$ and so $\left\|h_{m}-h_{n}\right\|_{V_{2}} \leq 2 M$.

To show (ii), notice that by (1) above,

$$
\left\|h_{m}-h_{n}\right\|_{\infty} \leq\left\|h_{n}-f\right\|_{\infty}+\left\|h_{m}-f\right\|_{\infty} \leq 2\|\widetilde{o s c}[0,1] f\|_{\infty}+\varepsilon_{n} \leq 4\|f\|_{\infty}+\varepsilon_{n} .
$$


For property (iii) observe that since $h_{m}-h_{n}$ is a continuous function on $[0,1]$, and $[0,1] \backslash D_{f}$ is dense in $[0,1]$, it suffices to check it for $\mathcal{I} \in \mathcal{F}\left([0,1] \backslash D_{f}\right)$. In this case, by Lemma 28 (ii), we have that

$$
\begin{aligned}
v_{2}^{2}\left(h_{m}-h_{n}, \mathcal{I}\right) & \leq 2 v_{2}^{2}\left(h_{m}-f, \mathcal{I}\right)+2 v_{2}^{2}\left(h_{n}-f, \mathcal{I}\right) \\
& \leq 4 \mu_{f}(\bigcup \mathcal{I})+32\|f\|_{V_{2}} \sqrt{\left\|\mu_{f}^{d}\right\|}+\varepsilon_{n} .
\end{aligned}
$$

Finally fix $k \in \mathbb{N}$ and let $V$ be an open subset of $[0,1]$ with $\mu_{f}(V)>\theta>0$. Choose a family $\left(I_{i}\right)_{i=1}^{m}$ of disjoint open intervals of $[0,1]$ such that $I_{i} \subseteq V$ and $\mu_{f}\left(\bigcup_{i=1}^{m} I_{i}\right)>\theta$ and let

$$
0<\varepsilon<\frac{\mu_{f}\left(\bigcup_{i=1}^{m} I_{i}\right)-\theta}{2(1+M) m} .
$$

Since $h_{k} \in V_{2}^{0}$, there is some $\delta>0$ such that

$$
\sup \left\{v_{2}\left(h_{k}, \mathcal{Q}\right): \mathcal{Q} \subseteq[0,1],\|\mathcal{Q}\|_{\max }<\delta\right\}<\varepsilon .
$$

For every $1 \leq i \leq m$, there exists $\mathcal{P}_{i} \subseteq I_{i}$ with $\left\|\mathcal{P}_{i}\right\|_{\max } \leq \delta$ and

$$
\mu_{f}\left(I_{i}\right)-\varepsilon<v_{2}^{2}\left(f, \mathcal{P}_{i}\right)
$$

Moreover since $\left(h_{n}\right)_{n}$ converges pointwise to $f$, there is $l>k$ such that for all $1 \leq i \leq m$,

$$
\left|v_{2}^{2}\left(f, \mathcal{P}_{i}\right)-v_{2}^{2}\left(h_{l}, \mathcal{P}_{i}\right)\right|<\varepsilon
$$

Then for every $1 \leq i \leq m$ we have that

$$
\begin{aligned}
v_{2}^{2}\left(h_{l}-h_{k}, \mathcal{P}_{i}\right) & \geq\left(v_{2}\left(h_{l}, \mathcal{P}_{i}\right)-v_{2}\left(h_{k}, \mathcal{P}_{i}\right)\right)^{2} \geq v_{2}^{2}\left(h_{l}, \mathcal{P}_{i}\right)-2 v_{2}\left(h_{k}, \mathcal{P}_{i}\right) v_{2}\left(h_{l}, \mathcal{P}_{i}\right) \\
& \geq v_{2}^{2}\left(f, \mathcal{P}_{i}\right)-\varepsilon-2 \varepsilon\left\|h_{l}\right\|_{V_{2}} \geq \mu_{f}\left(I_{i}\right)-2(1+M) \varepsilon
\end{aligned}
$$

Therefore, setting $\mathcal{J}=\bigcup_{i=1}^{k} \mathcal{I}_{\mathcal{P}_{i}}$, we obtain that

$$
v_{2}^{2}\left(h_{l}-h_{k}, \mathcal{J}\right)=\sum_{i=1}^{m} v_{2}^{2}\left(h_{l}-h_{k}, \mathcal{P}_{i}\right) \geq \sum_{i=1}^{m} \mu_{f}\left(I_{i}\right)-2(1+M) m \varepsilon>\theta .
$$

Remark 7. Notice that since $\ell_{1}$ is not embedded into $V_{2}^{0}$, from [18] and Goldstine's theorem, there is a sequence $\left(f_{n}\right)_{n}$ in $X$ pointwise converging to $f$ with $\left\|f_{n}\right\|_{V_{2}} \leq$ $\|f\|_{V_{2}}$. Hence, in Proposition 29 we can assume that $\left(f_{n}\right)_{n}$ (and thus also $\left.\left(h_{n}\right)_{n}\right)$ is a sequence in $X$ with $\left\|f_{n}\right\|_{V_{2}} \leq\|f\|_{V_{2}}=M$.

\section{On the Embedding of $c_{0}$ INTO Subspaces of $V_{2}^{0}$}

In this section we show that every subspace $X$ of $V_{2}^{0}$ with $X^{*}$ separable, $X^{* *}$ non-separable and $\mathcal{M}_{X^{* *}}=\left\{\mu_{f}: f \in X^{* *}\right\}$ separable contains an isomorphic copy of $c_{0}$. This is the first step towards the proof of the main theorem integrated in the next section.

\subsection{Sequences of $V_{2}^{0}$ dominated by measures.}

Definition 30. Let $\mu$ be a positive finite Borel measure on $[0,1]$ and $C, \varepsilon$ be positive constants. We will say that a function $G$ of $V_{2}^{0}$ is $(C, \varepsilon)$-dominated by $\mu$ if for every $\mathcal{I} \in \mathcal{A}$,

$$
v_{2}^{2}(G, \mathcal{I}) \leq C \mu(\bigcup \mathcal{I})+\varepsilon
$$


More generally for a sequence $\left(G_{n}\right)_{n}$ in $V_{2}^{0}$ and a sequence $\left(\varepsilon_{n}\right)_{n}$ of positive real numbers, we say that $\left(G_{n}\right)_{n}$ is $\left(C,\left(\varepsilon_{n}\right)_{n}\right)$-dominated by $\mu$ if for every $n \in \mathbb{N}$ and every $\mathcal{I} \in \mathcal{A}, v_{2}^{2}\left(G_{n}, \mathcal{I}\right) \leq C \mu(\bigcup \mathcal{I})+\varepsilon_{n}$.

Remark 8. Suppose that the sequence $\left(G_{n}\right)_{n}$ is $\left(C,\left(\varepsilon_{n}\right)_{n}\right)$-dominated by $\mu$ and $\sum_{n} \varepsilon_{n}=\varepsilon<\infty$. Then by the countable additivity and the monotonicity of $\mu$, it is easy to see that for every disjoint sequence $\left(\mathcal{I}_{n}\right)_{n}$ in $\mathcal{A}$, we have

$$
\sum_{n} v_{2}^{2}\left(G_{n}, \mathcal{I}_{n}\right) \leq C\|\mu\|+\varepsilon
$$

Proposition 31. Let $\left(G_{n}\right)_{n \in \mathbb{N}}$ be a sequence of functions of $V_{2}^{0}$ which is $\left(C,\left(\varepsilon_{n}\right)_{n}\right)$ dominated by a positive measure $\mu \in \mathcal{M}[0,1]$, for some null sequence $\left(\varepsilon_{n}\right)_{n}$ of positive real numbers. Assume also that $\left(G_{n}\right)_{n}$ is a seminormalized sequence in $V_{2}^{0}$ and $\lim _{n}\left\|G_{n}\right\|_{\infty}=0$. Then there is a subsequence of $\left(G_{n}\right)_{n \in \mathbb{N}}$ equivalent to the usual basis of $c_{0}$.

Proof. Let $0<\delta<\left\|G_{n}\right\|_{V_{2}} \leq M$. Since $\left(\varepsilon_{n}\right)_{n}$ is a null sequence, by passing to a subsequence we may assume that $\left(\varepsilon_{n}\right)_{n}$ is decreasing and $\sum_{n} \varepsilon_{n}=\varepsilon<\infty$. Since $\left(G_{n}\right)_{n}$ is seminormalized and pointwise convergent to zero, it is weakly null and so we may also suppose, by passing again to a subsequence, that it is a basic sequence. Also since $\lim _{n}\left\|G_{n}\right\|_{\infty}=0$, by Proposition 7 and passing to a further subsequence, we may suppose that $\left(G_{n}\right)_{n}$ is $\left(\varepsilon_{n}\right)_{n}$-biorthogonal.

As $\left(G_{n}\right)_{n}$ is a basic sequence, trivially $\left(G_{n}\right)_{n}$ has a lower $c_{0}$-estimate. To show that $\left(G_{n}\right)_{n}$ is dominated by the $c_{0}$-basis, let $\left(\lambda_{k}\right)_{k=1}^{n}$ be a sequence of scalars and let $\left|\lambda_{k_{0}}\right|=\max _{1 \leq k \leq n}\left|\lambda_{k}\right|$. Then by Lemma 11, we have that

$$
v_{2}^{2}\left(\sum_{k=1}^{n} \lambda_{k} G_{k}, \mathcal{I}\right) \leq\left|\lambda_{k_{0}}\right|^{2}\left(\sum_{k=1}^{n} v_{2}^{2}\left(G_{k}, \mathcal{I}^{(k)}\right)+\varepsilon(2 M+\varepsilon)\right),
$$

for every $\mathcal{I} \in \mathcal{A}$, which by (23) gives that

$$
v_{2}^{2}\left(\sum_{k=1}^{n} \lambda_{k} G_{k}, \mathcal{I}\right) \leq(C\|\mu\|+\varepsilon(2 M+1+\varepsilon))\left|\lambda_{k_{0}}\right|^{2} .
$$

Therefore setting $K=\sqrt{C\|\mu\|+\varepsilon(2 M+1+\varepsilon)}$, we conclude that

$$
\left\|\sum_{k=1}^{n} \lambda_{k} G_{k}\right\|_{V_{2}} \leq K \max _{1 \leq k \leq n}\left|\lambda_{k}\right|
$$

and the proof of the proposition is complete.

Remark 4 and the above proposition yield the following.

Corollary 32. The sequence $\left(R_{n}\right)_{n}$ defined in (13) contains a subsequence equivalent to the $c_{0}$-basis.

We also state the following generalization of Proposition 31 for later use.

Proposition 33. Let $\left(G_{n}\right)_{n \in \mathbb{N}}$ be a sequence of $V_{2}^{0},\left(\mu_{n}\right)_{n}$ be a sequence in $\mathcal{M}^{+}[0,1]$ and $\left(\varepsilon_{n}\right)_{n}$ be a null sequence of positive real numbers with the following properties.

(1) $\left(G_{n}\right)_{n}$ is a seminormalized sequence in $V_{2}^{0}$.

(2) $\lim _{n}\left\|G_{n}\right\|_{\infty}=0$. 
(3) There is a constant $C>0$ such that $G_{n}$ is $\left(C, \varepsilon_{n}\right)$-dominated by $\mu_{n}$, for all $n \in \mathbb{N}$.

(4) There is a measure $\mu \in \mathcal{M}^{+}[0,1]$ such that $\left(\mu_{n}\right)_{n}$ is norm convergent to $\mu$. Then there is a subsequence of $\left(G_{n}\right)_{n \in \mathbb{N}}$ equivalent to the usual basis of $c_{0}$.

Proof. By passing to a subsequence of $\left(G_{n}\right)_{n}$ we may suppose that for all $n \in \mathbb{N}$,

$$
\left\|\mu_{n}-\mu\right\|<2^{-n} \text {. }
$$

Let $0<\delta<\left\|G_{n}\right\|_{V_{2}} \leq M$. As in the proof of Proposition 31, by passing to a further subsequence we may also assume that $\sum_{n} \varepsilon_{n}=\varepsilon<\infty$ and that $\left(G_{n}\right)_{n}$ is again a basic sequence which in addition is $\left(\varepsilon_{n}\right)_{n}$-biorthogonal. To show that $\left(G_{n}\right)_{n}$ is dominated by the $c_{0}$-basis, equations (24) and (26) give that for every $\mathcal{I} \in \mathcal{A}$,

$$
\begin{aligned}
v_{2}^{2}\left(\sum_{k=1}^{n} \lambda_{k} G_{k}, \mathcal{I}\right) & \leq\left|\lambda_{k_{0}}\right|^{2}\left(C \sum_{k=1}^{n} \mu_{k}\left(\bigcup \mathcal{I}^{(k)}\right)+\varepsilon(2 M+1+\varepsilon)\right) \\
& \leq\left|\lambda_{k_{0}}\right|^{2}(C\|\mu\|+C+\varepsilon(2 M+1+\varepsilon)),
\end{aligned}
$$

which, setting $K=\sqrt{C\|\mu\|+C+\varepsilon(2 M+1+\varepsilon)}$, yields that

$$
\left\|\sum_{k=1}^{n} \lambda_{k} G_{k}\right\|_{V_{2}} \leq K \max \left\{\left|\lambda_{k}\right|: 1 \leq k \leq n\right\} \text {. }
$$

Proposition 34. Let $X$ be a subspace of $V_{2}^{0}$ and let $\left(f_{n}\right)_{n}$ be a bounded sequence in $X$ pointwise convergent to a function $f \in\left(X^{* *} \backslash X\right) \cap C[0,1]$. Then for every $0<\delta<\operatorname{dist}(f, X)$ and for every sequence $\left(\varepsilon_{n}\right)_{n}$ of positive real numbers there exists a convex block sequence $\left(h_{n}\right)_{n}$ of $\left(f_{n}\right)_{n}$ such that for all $n<m$ the following are satisfied:

(i) $\delta<\left\|h_{m}-h_{n}\right\|_{V_{2}} \leq 2 M$, where $M=\sup _{n}\left\|f_{n}\right\|_{V_{2}}$;

(ii) $\left\|h_{m}-h_{n}\right\|_{\infty} \leq \varepsilon_{n}$;

(iii) the function $h_{m}-h_{n}$ is $\left(4, \varepsilon_{n}\right)$-dominated by the measure $\mu_{f}$.

Proof. Since $f$ is continuous, we have that $\widetilde{o s c}_{[0,1]} f=0$ and $\mu_{f}^{d}=0$. The result now follows by Proposition 29.

Corollary 35. Let $X$ be a subspace of $V_{2}^{0}$ and $f \in\left(X^{* *} \backslash X\right) \cap C[0,1]$. Then $c_{0}$ is embedded into $X$.

Proof. As we have already mentioned (see Remark 7), there is a sequence $\left(f_{n}\right)_{n}$ in $X$ pointwise convergent to $f$ with $\left\|f_{n}\right\|_{V_{2}} \leq\|f\|_{V_{2}}$. Let $\left(\varepsilon_{n}\right)_{n}$ be a null sequence of positive real numbers and let $\left(h_{n}\right)_{n}$ be a convex block sequence of $\left(f_{n}\right)_{n}$ satisfying the properties of Proposition 34. For each $n \in \mathbb{N}$, let $G_{n}=h_{2 n}-h_{2 n-1}$. By (i)-(iii) of Proposition 34, we have that $\left(G_{n}\right)_{n}$ is a seminormalized sequence of functions in $X, \lim _{n}\left\|G_{n}\right\|_{\infty}=0$ and $\left(G_{n}\right)_{n}$ is $\left(4,\left(\varepsilon_{2 n-1}\right)_{n}\right)$-dominated by the measure $\mu_{f}$. By Proposition 31 the result follows.

Remark 9. Exploiting Lemma 34 more carefully, we may pass to an appropriate subsequence of $\left(h_{n}\right)_{n}$ which is equivalent to the summing basis. This gives an alternative proof of the known result that every $f \in V_{2} \cap C[0,1]$ is a difference

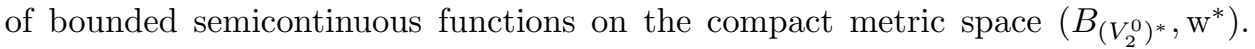
Moreover the converse of Corollary 35 also holds; that is, $\left(X^{* *} \backslash X\right) \cap C[0,1] \neq \emptyset$ if and only if $c_{0}$ is embedded into $X$ (cf. [3]). 


\subsection{The embedding of $c_{0}$ into $X$ when $\mathcal{M}_{X^{* *}}$ is separable.}

Lemma 36. Let $X$ be a subspace of $V_{2}^{0}$ and $\mathcal{F}$ be an uncountable subset of $X^{* *}$. If $D_{\mathcal{F}}=\bigcup_{f \in \mathcal{F}} D_{f}$ is countable, then for every $\varepsilon>0$ there is an uncountable subset $\mathcal{F}^{\prime} \subseteq \mathcal{F}$ such that for every $f_{1}, f_{2} \in \mathcal{F}^{\prime},\left\|\mu_{f_{1}-f_{2}}^{d}\right\|<\varepsilon$.

Proof. Let $D_{\mathcal{F}}=\left\{t_{n}\right\}_{n}$. By Proposition [16, we have that $\left\|\mu_{f}^{d}\right\|=\sum_{n} \tau_{f}\left(t_{n}\right) \leq$ $\|f\|_{V_{2}}^{2}$, for every $f \in \mathcal{F}$. By the definition of $\tau_{f}(t)$, we easily get the following inequalities.

(a) For every $f \in V_{2}, \tau_{f}(t) \leq 4\left(\left|f\left(t^{+}\right)\right|^{2}+\left|f\left(t^{+}\right)\right|^{2}+|f(t)|^{2}\right)$.

(b) For every $f_{1}, f_{2}$ in $V_{2}, \tau_{f_{1}-f_{2}}(t) \leq 2 \tau_{f_{1}}(t)+2 \tau_{f_{2}}(t)$.

By passing to an uncountable subset $\mathcal{F}^{\prime}$ of $\mathcal{F}$, we may suppose that the following hold.

(i) There is $n_{0} \in \mathbb{N}$ such that $\sum_{n>n_{0}} \tau_{f}\left(t_{n}\right)<\varepsilon / 8$, for all $f \in \mathcal{F}^{\prime}$.

(ii) For all $1 \leq n \leq n_{0}, j \in\{-, 0,+\}$ and $f_{1}, f_{2} \in \mathcal{F}^{\prime},\left|\left(f_{1}-f_{2}\right)\left(t_{n}^{j}\right)\right|<\sqrt{\frac{\varepsilon}{24 n_{0}}}$.

Then by (ii) and (a), we get that for every $f_{1}, f_{2} \in \mathcal{F}^{\prime}$,

$$
\sum_{n=1}^{n_{0}} \tau_{f_{1}-f_{2}}\left(t_{n}\right) \leq 4 \sum_{j \in\{-, 0,+\}} \sum_{n=1}^{n_{0}}\left|\left(f_{1}-f_{2}\right)\left(t_{n}^{j}\right)\right|^{2}<\varepsilon / 2 .
$$

Moreover by (i) and (b),

$$
\sum_{n>n_{0}} \tau_{f_{1}-f_{2}}\left(t_{n}\right) \leq 2 \sum_{n>n_{0}} \tau_{f_{1}}\left(t_{n}\right)+2 \sum_{n>n_{0}} \tau_{f_{2}}\left(t_{n}\right)<\varepsilon / 2 .
$$

Hence, by (27) and (28), $\left\|\mu_{f_{1}-f_{2}}^{d}\right\|=\sum_{n=1}^{n_{0}} \tau_{f_{1}-f_{2}}\left(t_{n}\right)+\sum_{n>n_{0}} \tau_{f_{1}-f_{2}}\left(t_{n}\right)<\varepsilon$.

Proposition 37. Let $X$ be a subspace of $V_{2}^{0}$ such that $X^{*}$ is separable, $X^{* *}$ nonseparable. If $\mathcal{M}_{X^{* *}}$ is a separable subset of $\mathcal{M}[0,1]$, then $c_{0}$ is embedded into $X$.

Proof. Let $\mathcal{F}$ be an uncountable subset of the unit sphere $S_{X^{* *}}$ of $X^{* *}$ such that for all $f_{1} \neq f_{2}$ in $\mathcal{F},\left\|f_{1}-f_{2}\right\|_{V_{2}}>3 \delta>0$. Since $X$ is separable, it is easy to see that by passing to a further uncountable subset, we may assume that for all $f_{1} \neq f_{2}$ in $\mathcal{F}$, dist $\left(f_{1}-f_{2}, X\right)>\delta$. Moreover since $X^{*}$ is separable, by Proposition 2 the set $D_{\mathcal{F}}$ is countable.

Let $\left(\varepsilon_{n}\right)_{n}$ be a sequence of positive real numbers with $\varepsilon=\sum_{n} \varepsilon_{n}<\infty$. Using Lemma 36. Proposition 4 and our assumption that $\mathcal{M}_{X^{* *}}$ is separable, we easily construct a decreasing sequence $\left(\mathcal{F}_{n}\right)_{n}$ of uncountable subsets of $\mathcal{F}$ such that

$$
\left\|\mu_{f_{1}-f_{2}}^{d}\right\|<\varepsilon_{n}^{2},\left\|f_{1}-f_{2}\right\|_{\infty}<\varepsilon_{n} \text { and }\left\|\mu_{f_{1}}-\mu_{f_{2}}\right\|<\varepsilon_{n}
$$

for all $n \in \mathbb{N}$ and $f_{1}, f_{2} \in \mathcal{F}_{n}$.

Given the above construction, we pick for each $n, f_{1}^{n} \neq f_{2}^{n}$ in $\mathcal{F}_{n}$. Notice that since $\left(\mathcal{F}_{n}\right)_{n}$ is decreasing, by (29) we have that $\left\|\mu_{f_{1}^{n}}-\mu_{f_{1}^{n+1}}\right\|<\varepsilon_{n}$ and so, as $\sum_{n} \varepsilon_{n}<\infty$, the sequence $\left(\mu_{f_{1}^{n}}\right)_{n}$ is norm-converging to a $\mu_{1} \in \mathcal{M}[0,1]$. Similarly $\left(\mu_{f_{2}^{n}}\right)_{n}$ converges to a $\mu_{2} \in \mathcal{M}[0,1]$. Let $F_{n}=f_{1}^{n}-f_{2}^{n}$. Applying for each $n \in \mathbb{N}$, Proposition 29 with $F_{n}$ in place of $f$, we obtain $G_{n} \in X$ satisfying the following:

(i) $\delta<\left\|G_{n}\right\|_{V_{2}} \leq 2\left\|F_{n}\right\|_{V_{2}} \leq 4$;

(ii) $\left\|G_{n}\right\|_{\infty} \leq 4\left\|F_{n}\right\|_{\infty}+\varepsilon_{n}$;

(iii) for every $\mathcal{I} \in \mathcal{A}, v_{2}^{2}\left(G_{n}, \mathcal{I}\right) \leq 4 \mu_{F_{n}}(\bigcup \mathcal{I})+32\left\|F_{n}\right\|_{V_{2}} \sqrt{\left\|\mu_{F_{n}}^{d}\right\|}+\varepsilon_{n}$. 
By (29), $\left\|F_{n}\right\|_{\infty}<\varepsilon_{n}$ and therefore (ii) gives

(iv) $\lim _{n}\left\|G_{n}\right\|_{\infty}=0$.

Moreover $\left\|\mu_{F_{n}}^{d}\right\|<\varepsilon_{n}^{2},\left\|F_{n}\right\|_{V_{2}} \leq 2$ and setting $\mu_{n}=2\left(\mu_{f_{1}^{n}}+\mu_{f_{2}^{n}}\right)$, by Proposition 18. $\mu_{F_{n}} \leq \mu_{n}$. Replacing in (iii) we get that for each $n \in \mathbb{N}$,

(v) for all $\mathcal{I} \in \mathcal{A}, v_{2}^{2}\left(G_{n}, \mathcal{I}\right) \leq 4 \mu_{n}(\bigcup \mathcal{I})+65 \varepsilon_{n}$, that is, $G_{n}$ is $\left(4,65 \varepsilon_{n}\right)$ dominated by $\mu_{n}$.

By (i), (iv), (v) and since $\left(\mu_{n}\right)_{n}$ is norm-convergent to $\mu_{1}+\mu_{2}$, the assumptions of Proposition 33 are fulfilled and so there is a subsequence of $\left(G_{n}\right)_{n}$ equivalent to the $c_{0}$-basis.

\section{On the EMBedding of $S^{2}$ INTO SUbSPaces OF $V_{2}^{0}$}

This final section includes the main results of the paper. We divide this section into three subsections. In the first subsection we define the $S^{2}$-systems and we show that their existence in a subspace $X$ of $V_{2}^{0}$ leads to the embedding of $S^{2}$ into $X$. A key ingredient is Lemma 41, which is of independent interest. In the next subsection we define the $S^{2}$-generating systems which provide the frame for building $S^{2}$-systems. Finally in the third subsection we show that every subspace $X$ of $V_{2}^{0}$ with $\mathcal{M}_{X^{* *}}$ non-separable contains an $S^{2}$-generating system and thus by the preceding results the space $S^{2}$ is embedded into $X$. We also show that $S^{2}$ is contained into $T F$.

6.1. $S^{2}$-systems. In this subsection we will define certain structures closely related with the embedding of the space $S^{2}$ into $V_{2}^{0}$. We start with the definition of a system.

Definition 38. Let $M, \Lambda, \theta$ be positive constants and $\left(\varepsilon_{n}\right)_{n=0}^{\infty}$ be a sequence of positive real numbers. An $\left(\varepsilon_{n}\right)_{n}$-system with constants $(M, \Lambda, \theta)$ is a family of the form

$$
\left(\left(G_{s}, \nu_{s}, \mathcal{I}_{s}\right)_{s \in 2<\mathbb{N}},\left(\mathcal{Q}_{n}\right)_{n \in \mathbb{N}}\right)
$$

where $\left(G_{s}\right)_{s \in 2<\mathbb{N}}$ is a family of functions of $V_{2}^{0},\left(\nu_{s}\right)_{s \in 2<\mathbb{N}}$ is a family of positive Borel measures on $[0,1],\left(\mathcal{I}_{s}\right)_{s \in 2<\mathbb{N}}$ is a family in $\mathcal{A}$, and $\left(\mathcal{Q}_{n}\right)_{n \in \mathbb{N}}$ is an increasing sequence of finite subsets of $[0,1]$, satisfying the following properties.

(1) For every $s \in 2^{<\mathbb{N}},\left\|G_{s}\right\|_{V_{2}} \leq M$ and $\left\|\nu_{s}\right\| \leq \Lambda$.

(2) For every $n \geq 0$ and $s \in 2^{n},\left\|G_{s}\right\|_{\infty} \leq \varepsilon_{n}$.

(3) For every $n \geq 0$, the set $\mathcal{Q}_{n} \varepsilon_{n}$-determines the quadratic variation of $\left\langle\left\{G_{s}\right.\right.$ : $\left.\left.s \in 2^{n}\right\}\right\rangle$.

(4) For every $n \geq 0, s \in 2^{n}$ and every $\mathcal{I} \in \mathcal{F}\left(\mathcal{Q}_{n}\right), v_{2}^{2}\left(G_{s}, \mathcal{I}\right) \leq \nu_{s}(\bigcup \mathcal{I})+\varepsilon_{n}$.

(5) For every $s \perp t,\left(\mathcal{I}_{s}, \mathcal{I}_{t}\right)$ is a disjoint pair.

(6) For every $s \in 2^{<\mathbb{N}}, v_{2}^{2}\left(G_{s}, \mathcal{I}_{s}\right)>\theta$.

Remark 10. Notice that by property (2), we have that $\lim \left\|G_{\sigma \mid n}\right\|_{\infty}=0$ and therefore by Proposition 9 for every family $\left(\varepsilon_{s}\right)_{s}$ of positive scalars there is a dyadic subtree $\left(t_{s}\right)_{s}$ such that $\left(G_{t_{s}}\right)_{s}$ is $\left(\varepsilon_{s}\right)_{s}$-biorthogonal. Moreover by (3) and (4) we have that for every $s \in 2^{n}$, the function $G_{s}$ is $\left(1,2 \varepsilon_{n}\right)$-dominated by $\nu_{s}$.

Definition 39. An $\left(\varepsilon_{n}\right)_{n}-S^{2}$ system with constants $(M, \Lambda, \theta)$ is an $\left(\varepsilon_{n}\right)_{n}-$ system $\left(\left(G_{s}, \nu_{s}, \mathcal{I}_{s}\right)_{s \in 2<\mathbb{N}},\left(\mathcal{Q}_{n}\right)_{n}\right)$, with the same constants satisfying in addition the following property. For every $0 \leq n \leq m, s \in 2^{n}, t \in 2^{m}$ with $s \sqsubseteq t$ and $\mathcal{I} \in \mathcal{F}\left(\mathcal{Q}_{n}\right)$,

$$
\left|\nu_{t}(\bigcup \mathcal{I})-\nu_{s}(\bigcup \mathcal{I})\right|<\varepsilon_{n}
$$


Remark 11. Suppose that $\left(\varepsilon_{n}\right)_{n}$ is a null sequence. Then, as $\mathcal{Q}_{n}$ is increasing, by (30) we get that for every $\sigma \in 2^{\mathbb{N}}$ and every $\mathcal{I} \in \mathcal{F}\left(\cup_{n} \mathcal{Q}_{n}\right)$, the sequence $\left(\nu_{\sigma \mid n}(\bigcup \mathcal{I})\right)_{n}$ is Cauchy.

Lemma 40. Let $\left(\left(G_{s}, \nu_{s}, \mathcal{I}_{s}\right)_{s \in 2<\mathbb{N}}\left(\mathcal{Q}_{n}\right)_{n \in \mathbb{N}}\right)$, be an $\left(\varepsilon_{n}\right)_{n}-S^{2}$ system with constants $(M, \Lambda, \theta)$. Assume also that $\left(\varepsilon_{n}\right)_{n}$ is a null sequence. Then there exists a family of positive Borel measures $\left(\nu_{\sigma}\right)_{\sigma \in 2^{\mathbb{N}}}$ on $[0,1]$ such that $\sup _{\sigma}\left\|\nu_{\sigma}\right\| \leq \Lambda$, and for all $\sigma \in 2^{\mathbb{N}},\left(G_{\sigma \mid n}\right)_{n}$ is $\left(1,\left(3 \varepsilon_{n}\right)_{n}\right)$-dominated by $\nu_{\sigma}$.

Proof. Let $\sigma \in 2^{\mathbb{N}}$. Since $\left(\nu_{\sigma \mid n}\right)_{n}$ is a bounded sequence in $\mathcal{M}[0,1]$, there exist a subsequence $\left(\nu_{\sigma \mid n}\right)_{n \in L}$ and a positive Borel measure $\nu_{\sigma}$ on $[0,1]$, such that $\left(\nu_{\sigma \mid n}\right)_{n \in L}$ is $\mathrm{w}^{*}-$ convergent to $\nu_{\sigma}$. For the following, fix $k \geq 0$ and a finite family of intervals $\mathcal{I} \in \mathcal{A}$. By condition (3) of Definition 38, there exists $\widetilde{\mathcal{I}} \in \mathcal{F}\left(\mathcal{Q}_{k}\right)$ with $\widetilde{\mathcal{I}} \preceq \mathcal{I}$ such that $\left|v_{2}^{2}\left(G_{\sigma \mid k}, \mathcal{I}\right)-v_{2}^{2}\left(G_{\sigma \mid k}, \widetilde{\mathcal{I}}\right)\right|<\varepsilon_{k}$. Moreover by condition (4) of Definition 38 and (30), we get that for every $m>k$,

$$
v_{2}^{2}\left(G_{\sigma \mid k}, \mathcal{I}\right) \leq v_{2}^{2}\left(G_{\sigma \mid k}, \tilde{\mathcal{I}}\right)+\varepsilon_{k} \leq \nu_{\sigma \mid k}(\bigcup \tilde{\mathcal{I}})+2 \varepsilon_{k} \leq \nu_{\sigma \mid m}(\bigcup \tilde{\mathcal{I}})+3 \varepsilon_{k}
$$

By Remark 11, we have that $\lim _{m} \nu_{\sigma \mid m}(\bigcup \widetilde{\mathcal{I}})$ exists and so (31) implies that

$$
v_{2}^{2}\left(G_{\sigma \mid k}, \mathcal{I}\right) \leq \lim _{m} \nu_{\sigma \mid m}(\bigcup \widetilde{\mathcal{I}})+3 \varepsilon_{k}=\lim _{n \in L} \nu_{\sigma \mid n}(\bigcup \widetilde{\mathcal{I}})+3 \varepsilon_{k}
$$

As the set $\bigcup \widetilde{\mathcal{I}}$ is a closed subset of $[0,1]$ and $\mathrm{w}^{*}-\lim _{n \in L} \nu_{\sigma \mid n}=\nu_{\sigma}$, by Portmanteau's theorem (see [12, page 111) and the monotonicity of $\nu_{\sigma}$, we have that

$$
\lim _{n \in L} \nu_{\sigma \mid n}(\bigcup \tilde{\mathcal{I}}) \leq \nu_{\sigma}(\bigcup \tilde{\mathcal{I}}) \leq \nu_{\sigma}(\bigcup \mathcal{I})
$$

By (32) and (33) we conclude that for every $k \geq 0$ and every $\mathcal{I} \in \mathcal{A}$,

$$
v_{2}^{2}\left(G_{\sigma \mid k}, \mathcal{I}\right) \leq \nu_{\sigma}(\bigcup \mathcal{I})+3 \varepsilon_{k}
$$

that is, the sequence $\left(G_{\sigma \mid n}\right)_{n}$ is $\left(1,\left(3 \varepsilon_{n}\right)_{n}\right)$-dominated by the measure $\nu_{\sigma}$. Finally since $\nu_{\sigma}$ is in the $\mathrm{w}^{*}$-closure of $\left\{\nu_{s}\right\}_{s \in 2}<\mathbb{N},\left\|\nu_{\sigma}\right\| \leq \sup _{n}\left\|\nu_{\sigma \mid n}\right\| \leq \Lambda$.

Remark 12. Notice that if $\sum_{n} \varepsilon_{n}=\epsilon<\infty$, the above lemma yields that for every $\sigma \in 2^{\mathbb{N}}$ and every disjoint family $\left(\mathcal{I}_{n}\right)_{n}$ in $\mathcal{A}$, we have that

$$
\sum_{n} v_{2}^{2}\left(G_{\sigma \mid n}, \mathcal{I}_{n}\right) \leq \Lambda+3 \epsilon
$$

The next lemma concerns an inequality for tree families of positive numbers which is critical for the embedding of $S^{2}$ into subspaces of $V_{2}^{0}$ and could be useful elsewhere.

Lemma 41. Let $\left(\alpha_{s}\right)_{s \in 2<\mathbb{N}},\left(\lambda_{s}\right)_{s \in 2<\mathbb{N}}$ be two families of non-negative real numbers and let $n \geq 0$. Then there exists a maximal antichain $A$ of $2^{\leqslant n}$ and a family of branches $\left(b_{t}\right)_{t \in A}$ of $2^{\leqslant n}$ such that $\sum_{s \in 2 \leqslant n} \lambda_{s} \alpha_{s} \leq \sum_{t \in A}\left(\sum_{s \in b_{t}} \alpha_{s}\right) \lambda_{t}$ and $t \in b_{t}$, for all $t \in A$. Therefore if $\sum_{n=1}^{\infty} \alpha_{\sigma \mid n} \leq C$, for all $\sigma \in 2^{\mathbb{N}}$, then for each $n \geq 0$ there is an antichain $A$ of $2^{\leqslant n}$ such that $\sum_{s \in 2 \leqslant n} \lambda_{s} \alpha_{s} \leq C \sum_{s \in A} \lambda_{s}$.

Proof. We shall use induction on $n \geq 0$. The lemma trivially holds for $n=0$. Assuming that it is true for some $n$, we show the $n+1$ case. For each $j \in\{0,1\}$, let $\mathcal{D}_{j}=\left\{t \in 2^{n+1}: t(1)=j\right\}$. Then $\mathcal{D}_{j}$ is order isomorphic to $2^{\leqslant n}$ and so by our 
inductive assumption there is an antichain $A_{j} \subseteq \mathcal{D}_{j}$, and a family of branches $\left\{b_{t}^{j}\right.$ : $\left.t \in A_{j}\right\} \subseteq \mathcal{D}_{j}$ with $t \in b_{t}^{j}$, for each $t \in A_{j}$ and $\sum_{s \in \mathcal{D}_{j}} \lambda_{s} \alpha_{s} \leq \sum_{t \in A_{j}}\left(\sum_{s \in b_{t}^{j}} \alpha_{s}\right) \lambda_{t}$. Hence we easily get that

$$
\sum_{s \in 2 \leqslant n+1} \lambda_{s} \alpha_{s}=\lambda_{\emptyset} \alpha_{\emptyset}+\sum_{t \in \mathcal{D}_{0} \cup \mathcal{D}_{1}} \lambda_{t} \alpha_{t} \leq \lambda_{\emptyset} \alpha_{\emptyset}+\sum_{t \in A_{0}}\left(\sum_{s \in b_{t}^{0}} \alpha_{s}\right) \lambda_{t}+\sum_{t \in A_{1}}\left(\sum_{s \in b_{t}^{1}} \alpha_{s}\right) \lambda_{t} .
$$

We distinguish two cases.

Case 1. $\lambda_{\emptyset} \leq \sum_{t \in A_{0}} \lambda_{t}+\sum_{t \in A_{1}} \lambda_{t}$. Then let $A=A_{0} \cup A_{1}$ and $b_{t}=b_{t}^{j} \cup\{\emptyset\}$, for each $t \in A_{j}$. Obviously $A$ is a maximal antichain in $2^{\leqslant n+1}$ and $\left\{b_{t}: t \in A\right\}$ is a family of branches with $t \in b_{t}$ for each $t \in A$. Moreover as $\lambda_{\emptyset} \leq \sum_{t \in A} \lambda_{t}$, by (36) we obtain that

$$
\sum_{s \in 2 \leqslant n+1} \lambda_{s} \alpha_{s} \leq \sum_{t \in A}\left(\sum_{s \in b_{t}} \alpha_{s}\right) \lambda_{t} .
$$

Case 2. $\sum_{t \in A_{0}} \lambda_{t}+\sum_{t \in A_{1}} \lambda_{t}<\lambda_{\emptyset}$. Then we set $A=\{\emptyset\}$ and $b_{\emptyset}=\{\emptyset\} \cup b_{t_{0}}^{j_{0}}$ where $\sum_{s \in b_{t_{0}}^{j_{0}}} \alpha_{s}=\max \bigcup_{j=0}^{1}\left\{\sum_{s \in b_{t}^{j}} \alpha_{s}: t \in A_{j}\right\}$. By (36), we get that

$$
\sum_{s \in 2 \leqslant n+1} \lambda_{s} \alpha_{s} \leq \lambda_{\emptyset}\left(\alpha_{\emptyset}+\sum_{s \in b_{t_{0}}^{j_{0}}} \alpha_{s}\right) \leq\left(\sum_{s \in b_{\emptyset}} \alpha_{s}\right) \lambda_{\emptyset} .
$$

Proposition 42. Let $\left(\left(G_{s}, \nu_{s}, \mathcal{I}_{s}\right)_{s \in 2^{<N}},\left(\mathcal{Q}_{n}\right)_{n}\right)$, be an $\left(\varepsilon_{n}\right)_{n}-S^{2}$ system with constants $(M, \Lambda, \theta)$. Suppose that $\left(\varepsilon_{n}\right)_{n}$ is a summable sequence of positive real numbers. Then there is a dyadic subtree $\left(t_{s}\right)_{s \in 2^{n}}$ of $2^{<\mathbb{N}}$ such that $\left(G_{t_{s}}\right)_{s \in 2^{<\mathbb{N}}}$ is equivalent to the $S^{2}$-basis.

Proof. Let $\left(\varepsilon_{s}\right)_{s \in 2<\mathbb{N}}$ be a family of positive real numbers such that $\sum_{s} \varepsilon_{s}=\varepsilon<\infty$ and $\theta-(\varepsilon+2 M) \varepsilon>0$. As we have already mentioned (see Remark 10), there is a

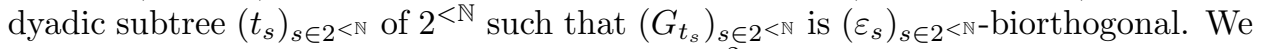
will show that $\left(G_{t_{s}}\right)_{s \in 2<\mathbb{N}}$ is equivalent to the $S^{2}$-basis. To this end, fix a sequence of real numbers $\left(\lambda_{s}\right)_{|s| \leq n}$.

First we show the upper $S^{2}$-estimate. Let $\mathcal{I} \in \mathcal{A}$. By Lemma 11 we have that

$$
v_{2}^{2}\left(\sum_{|s| \leq n} \lambda_{s} G_{t_{s}}, \mathcal{I}\right) \leq \sum_{|s| \leq n}\left|\lambda_{s}\right|^{2} v_{2}^{2}\left(G_{t_{s}}, \mathcal{I}^{\left(t_{s}\right)}\right)+\max _{|s| \leq n}\left|\lambda_{s}\right|^{2}(2 M+\varepsilon) \varepsilon .
$$

By Remark 12, we have that $\sum_{n} v_{2}^{2}\left(G_{t_{\sigma \mid n}}, \mathcal{I}^{\left(t_{\sigma \mid n}\right)}\right) \leq \Lambda+3 \epsilon$, where $\epsilon=\sum_{n} \varepsilon_{n}$. Hence by Lemma 41 (with $v_{2}^{2}\left(G_{t_{s}}, \mathcal{I}^{\left(t_{s}\right)}\right)$ and $\left|\lambda_{s}\right|^{2}$ in place of $\alpha_{s}$ and $\left|\lambda_{s}\right|$, respectively), we obtain an antichain $A \subseteq 2^{\leqslant n}$ such that

$$
\sum_{|s| \leq n}\left|\lambda_{s}\right|^{2} v_{2}^{2}\left(G_{t_{s}}, \mathcal{I}^{\left(t_{s}\right)}\right) \leq(\Lambda+3 \epsilon) \sum_{s \in A}\left|\lambda_{s}\right|^{2}
$$

By (37) and (38), we get that

$$
\begin{aligned}
v_{2}^{2}\left(\sum_{|s| \leq n} \lambda_{s} G_{t_{s}}, \mathcal{I}\right) & \leq(\Lambda+3 \epsilon) \sum_{s \in A}\left|\lambda_{s}\right|^{2}+\max _{|s| \leq n}\left|\lambda_{s}\right|^{2}(2 M+\varepsilon) \varepsilon \\
& \leq(\Lambda+3 \epsilon+(2 M+\varepsilon) \varepsilon)\left\|\sum_{|s| \leq n} \lambda_{s} e_{s}\right\|_{S^{2}}^{2} .
\end{aligned}
$$

This yields that there is $C>0$ such that $\left\|\sum_{|s| \leq n} \lambda_{s} G_{t_{s}}\right\|_{V_{2}} \leq C\left\|\sum_{|s| \leq n} \lambda_{s} e_{s}\right\|_{S^{2}}$. 
We now proceed to show the lower $S^{2}$-estimate. Let $A$ be an antichain of $2^{\leqslant n}$ such that

$$
\left\|\sum_{|s| \leq n} \lambda_{s} e_{s}\right\|_{S^{2}}=\left(\sum_{s \in A}\left|\lambda_{s}\right|^{2}\right)^{1 / 2}
$$

Since $\left(\mathcal{I}_{t_{s}}\right)_{s \in A}$ is a disjoint family, we get that $\mathcal{I}=\bigcup_{s \in A} \mathcal{I}_{t_{s}} \in \mathcal{A}$. By Lemma 11 and (39), we have that

$$
\begin{aligned}
v_{2}^{2}\left(\sum_{|s| \leq n} \lambda_{s} G_{t_{s}}, \mathcal{I}\right) & \geq \sum_{s \in A}\left|\lambda_{s}\right|^{2} v_{2}^{2}\left(G_{t_{s}}, \mathcal{I}^{\left(t_{s}\right)}\right)-\max _{|s| \leq n}\left|\lambda_{s}\right|^{2}\left(\sum_{|s| \leq n} \varepsilon_{s}\right) 2 M \\
& \geq \sum_{s \in A}\left|\lambda_{s}\right|^{2} v_{2}^{2}\left(G_{t_{s}}, \mathcal{I}^{\left(t_{s}\right)}\right)-\left(\sum_{s \in A}\left|\lambda_{s}\right|^{2}\right) 2 M \varepsilon
\end{aligned}
$$

By the properties of the $S^{2}$-system, we have that for every $s \in A$,

$\theta<v_{2}^{2}\left(G_{t_{s}}, \mathcal{I}_{t_{s}}\right) \leq v_{2}^{2}\left(G_{t_{s}}, \mathcal{I}\right)=v_{2}^{2}\left(G_{t_{s}}, \mathcal{I}^{\left(t_{s}\right)}\right)+v_{2}^{2}\left(G_{t_{s}}, \mathcal{I} \backslash \mathcal{I}^{\left(t_{s}\right)}\right) \leq v_{2}^{2}\left(G_{t_{s}}, \mathcal{I}^{\left(t_{s}\right)}\right)+\varepsilon^{2}$ and therefore $v_{2}^{2}\left(G_{t_{s}}, \mathcal{I}^{\left(t_{s}\right)}\right) \geq \theta-\varepsilon^{2}$. Hence by (40), we obtain that

$$
v_{2}^{2}\left(\sum_{|s| \leq n} \lambda_{s} G_{t_{s}}, \mathcal{I}\right) \geq(\theta-(\varepsilon+2 M) \varepsilon)\left(\sum_{s \in A}\left|\lambda_{s}\right|^{2}\right)
$$

which gives that there is $c>0$ such that $\left\|\sum_{|s| \leq n} \lambda_{s} G_{t_{s}}\right\|_{V_{2}} \geq c\left\|\sum_{|s| \leq n} \lambda_{s} e_{s}\right\|_{S^{2}}$.

\section{2. $S^{2}$-generating systems.}

Definition 43. An $\left(\varepsilon,\left(\varepsilon_{n}\right)_{n}\right)-S^{2}$-generating system with constants $(M, \Lambda, \theta)$ is an $\left(\varepsilon_{n}\right)_{n}$-system $\left(\left(H_{s}, \mu_{s}, \mathcal{J}_{s}\right)_{s \in 2<\mathrm{N}},\left(\mathcal{P}_{n}\right)_{n}\right)$, with the same constants satisfying in addition the following properties.

(i) For every $m>n \geq 0, s \in 2^{n}$ and $\left\{s_{0}, s_{1}\right\} \subseteq 2^{m}$ such that $s^{\frown} 0 \sqsubseteq s_{0}$ and $s^{\wedge} 1 \sqsubseteq s_{1}$ and every $\mathcal{I} \in \mathcal{F}\left(\mathcal{P}_{n}\right)$, we have that

$$
\left|\frac{\mu_{s_{0}}+\mu_{s_{1}}}{2}(\bigcup \mathcal{I})-\mu_{s}(\bigcup \mathcal{I})\right|<\varepsilon_{n}
$$

(ii) For every $n \geq 1$, the sequence $\left(H_{s}\right)_{s \in 2^{n}}$ is $\left(\varepsilon_{s}\right)_{s \in 2^{n}}$-biorthogonal, where $\varepsilon=\sum_{s \in 2^{n}} \varepsilon_{s}>0$

In the following we present a partition of $2^{<\mathbb{N}}$ in continuum many almost disjoint subtrees. (Recall that a family of countable sets is called almost disjoint if the intersection of any two members of the family is finite.) This partition is induced by the canonical bijection between $2^{<\mathbb{N}}$ and $2^{<\mathbb{N}} \times 2^{<\mathbb{N}}$.

Definition 44. Let $s \in 2^{<\mathbb{N}}$. If $s=\emptyset$, then let $L_{\emptyset}=\{\emptyset\}$. If $\emptyset \neq s=(s(1), \ldots, s(n))$, let $L_{s}=\left\{t \in 2^{2 n}: t(2 i)=s(i)\right.$, for all $\left.1 \leq i \leq n\right\}$.

Remark 13. It is easy to see that for each $\sigma \in 2^{\mathbb{N}}, T_{\sigma}=\bigcup_{n} L_{\sigma \mid n}$ is a dyadic subtree of $2^{<\mathbb{N}}$ and $\left(T_{\sigma}\right)_{\sigma \in 2^{\mathbb{N}}}$ is an almost disjoint family and hence their bodies $\left(\left[T_{\sigma}\right]\right)_{\sigma \in 2^{\mathbb{N}}}$ are disjoint. (Recall that the body of a tree $T$ in $2^{<\mathbb{N}}$ is defined to be the set $[T]=\left\{\sigma \in 2^{\mathbb{N}}: \forall n(\sigma \mid n \in T)\right\}$ (see also [12], page 5).)

The following properties of $\left(L_{s}\right)_{s \in 2^{<\mathbb{N}}}$ are easily established.

(L1) For all $n \geq 0$ and $s \in 2^{n}, L_{s} \subseteq 2^{2 n}$ and $\left|L_{s}\right|=2^{n}$, where $\left|L_{s}\right|$ is the cardinality of $L_{s}$.

(L2) For $s_{1} \sqsubseteq s_{2}, L_{s_{1}}=L_{s_{2}} \mid n_{1}=\left\{t \mid n_{1}: t \in L_{s_{2}}\right\}$, where $n_{1}=2\left|s_{1}\right|$. 
(L3) For $s_{1} \perp s_{2}, L_{s_{1}} \perp L_{s_{2}}$.

(L4) For all $n \geq 0,2^{2 n}=\bigcup_{s \in 2^{n}} L_{s}$.

Given an $\left(\varepsilon,\left(\varepsilon_{n}\right)_{n}\right)-S^{2}$-generating system $\left(\left(H_{s}, \mu_{s}, \mathcal{J}_{s}\right)_{s \in 2<\mathbb{N}},\left(\mathcal{P}_{n}\right)_{n}\right)$, with constants $(M, \Lambda, \theta)$, we set $G_{s}=2^{-n / 2} \sum_{t \in L_{s}} H_{t}, \nu_{s}=2^{-n} \sum_{t \in L_{s}} \mu_{t}, \mathcal{I}_{s}=\bigcup_{t \in L_{s}} \mathcal{J}_{t}$, $\mathcal{Q}_{n}=\mathcal{P}_{2 n}$ and $\varepsilon_{n}^{\prime}=\frac{\theta}{2^{n / 2}}+2^{n / 2} \varepsilon_{2 n}$.

Proposition 45. If $\theta^{\prime}=\theta-(2 M+\varepsilon) \varepsilon>0$, then the system $\left(\left(G_{s}, \nu_{s}, \mathcal{I}_{s}\right)_{s \in 2^{\mathbb{N}}},\left(\mathcal{Q}_{n}\right)_{n}\right)$ is an $\left(\varepsilon_{n}^{\prime}\right)_{n}-S^{2}$-system with constants $\left(M+\varepsilon, \Lambda, \theta^{\prime}\right)$.

Proof. Let $n \geq 1$ and $s \in 2^{n}$. Since $L_{s} \subseteq 2^{2 n},\left|L_{s}\right|=2^{n}$ and $\left(H_{t}\right)_{t \in 2^{2 n}}$ is $\left(\varepsilon_{t}\right)_{t \in 2^{2 n} \text { - }}$ biorthogonal, with $\sum_{t \in 2^{2 n}} \varepsilon_{t}=\varepsilon<1$, by Lemma [11, we get that for every $\mathcal{I} \in \mathcal{A}$,

$$
v_{2}^{2}\left(G_{s}, \mathcal{I}\right)<\left|L_{s}\right|^{-1} \sum_{t \in L_{s}} v_{2}^{2}\left(H_{t}, \mathcal{I}^{(t)}\right)+2^{-n}(2 M+\varepsilon) \varepsilon \leq(M+\varepsilon)^{2}
$$

and so $\left\|G_{s}\right\|_{V_{2}^{0}} \leq M+\varepsilon$.

Moreover $\left\|G_{s}\right\|_{\infty} \leq \sqrt{\left|L_{s}\right|} \varepsilon_{2 n}=\sqrt{2^{n}} \varepsilon_{2 n}<\varepsilon_{n}^{\prime}$. We now show that the quadratic variation of $\left\langle\left\{G_{s}\right\}_{s \in 2^{n}}\right\rangle$ is $\varepsilon_{n}^{\prime}$-determined by $\mathcal{Q}_{n}$. Let $\mathcal{I} \in \mathcal{A}$. Since $\mathcal{P}_{2 n} \varepsilon_{2 n^{-}}$ determines the quadratic variation of $\left\langle\left\{H_{t}\right\}_{t \in 2^{2 n}}\right\rangle$, there is $\widetilde{\mathcal{I}} \preceq \mathcal{I}$ in $\mathcal{F}\left(\mathcal{P}_{2 n}\right)$ such that for all $\left(\mu_{t}\right)_{t \in 2^{2 n}}$,

$$
\left|v_{2}^{2}\left(\sum_{t \in 2^{2 n}} \mu_{t} H_{t}, \mathcal{I}\right)-v_{2}^{2}\left(\sum_{t \in 2^{2 n}} \mu_{t} H_{t}, \widetilde{\mathcal{I}}\right)\right| \leq\left(\sum_{t \in 2^{2 n}}\left|\mu_{t}\right|^{2}\right) \varepsilon_{2 n} .
$$

Notice that for every sequence of scalars $\left(\lambda_{s}\right)_{s \in 2^{n}}, \sum_{s \in 2^{n}} \lambda_{s} G_{s}=\sum_{t \in 2^{2 n}} \mu_{t} H_{t}$, where for each $t \in 2^{2 n}, \mu_{t}=\left|L_{s}\right|^{-1 / 2} \lambda_{s}$, for $t \in L_{s}$. Therefore

$$
\begin{aligned}
\left|v_{2}^{2}\left(\sum_{s \in 2^{n}} \lambda_{s} G_{s}, \mathcal{I}\right)-v_{2}^{2}\left(\sum_{s \in 2^{n}} \lambda_{s} G_{s}, \widetilde{\mathcal{I}}\right)\right| & =\left|v_{2}^{2}\left(\sum_{t \in 2^{2 n}} \mu_{t} H_{t}, \mathcal{I}\right)-v_{2}^{2}\left(\sum_{t \in 2^{2 n}} \mu_{t} H_{t}, \widetilde{\mathcal{I}}\right)\right| \\
& \leq\left(\sum_{t \in 2^{2 n}}\left|\mu_{t}\right|^{2}\right) \varepsilon_{2 n} \leq\left(\sum_{s \in 2^{n}}\left|\lambda_{s}\right|^{2}\right) \varepsilon_{n}^{\prime} .
\end{aligned}
$$

We proceed to show property (4) of Definition 38, Let $\mathcal{I} \in \mathcal{F}\left(\mathcal{Q}_{n}\right)$. Then $\mathcal{I} \in$ $\mathcal{F}\left(\mathcal{P}_{2 n}\right), \mathcal{I}=\bigcup_{t \in 2^{2 n}} \mathcal{I}^{(t)}$ and for all $t \in 2^{2 n}, v_{2}^{2}\left(H_{t}, \mathcal{I}^{(t)}\right) \leq \mu_{t}\left(\bigcup \mathcal{I}^{(t)}\right)+\varepsilon_{2 n}$. Notice also that for every $t \in L_{s}, \mu_{t} \leq\left|L_{s}\right| \nu_{s}$. Hence, by Lemma 11, we get that

$$
\begin{aligned}
v_{2}^{2}\left(G_{s}, \mathcal{I}\right) & \leq\left|L_{s}\right|^{-1} \sum_{t \in L_{s}} \mu_{t}\left(\bigcup \mathcal{I}^{(t)}\right)+\varepsilon_{2 n}+2^{-n}(2 M+\varepsilon) \varepsilon \\
& \leq \sum_{t \in L_{s}} \nu_{s}\left(\bigcup \mathcal{I}^{(t)}\right)+\varepsilon_{2 n}+3 \theta /\left(4 \sqrt{2^{n}}\right) \leq \nu_{s}(\bigcup \mathcal{I})+\varepsilon_{n}^{\prime} .
\end{aligned}
$$

We now show (30) of Definition 39, Let $m \geq 1$ and $t=(t(1), \ldots, t(m)) \in 2^{m}$. Let $0 \leq n<m$ and $s=t \mid n$. For each $w \in L_{s}$, let $w_{0}=w^{\curvearrowleft} 0^{\frown} t(n+1)$ and $w_{1}=w^{\wedge} 1^{\frown} t(n+1)$. Also let $u=(u(1), \ldots, u(m-n-1))$, where $u(i)=t(n+1+i)$, for all $1 \leq i \leq m-n-1$. Then $L_{t}=\bigcup_{w \in L_{s}}\left(w_{0}^{\curlyvee} L_{u} \cup w_{1}^{\curlyvee} L_{u}\right)$ and so $\nu_{t}=$ $2^{-m} \sum_{w \in L_{s}} \sum_{v \in L_{u}}\left(\mu_{w_{0}^{-} v}+\mu_{w_{1}^{-} v}\right)$. Therefore,

$$
\begin{aligned}
\nu_{t}-\nu_{s} & =2^{-m} \sum_{w \in L_{s}} \sum_{v \in L_{u}}\left(\mu_{w_{0}^{-} v}+\mu_{w_{1}^{-} v}\right)-2^{-n} \sum_{w \in L_{s}} \mu_{w} \\
& =2^{-m} \sum_{w \in L_{s}} \sum_{v \in L_{u}}\left(\mu_{w_{0}^{-} v}+\mu_{w_{1}^{-} v}-2 \mu_{w}\right) .
\end{aligned}
$$


Moreover by (41), for every $\mathcal{I} \in \mathcal{F}\left(\mathcal{Q}_{n}\right)=\mathcal{F}\left(\mathcal{P}_{2 n}\right)$, and all $v \in L_{u}$, we have that

$$
\left|\left(\mu_{w_{0}^{-} v}+\mu_{w_{1}^{\curvearrowright} v}-2 \mu_{w}\right)(\bigcup \mathcal{I})\right| \leq 2 \varepsilon_{2 n} .
$$

Hence $\left|\nu_{t}(\bigcup \mathcal{I})-\nu_{s}(\bigcup \mathcal{I})\right| \leq 2^{-m} 2^{n} 2^{m-n-1} 2 \varepsilon_{2 n}=\varepsilon_{2 n} \leq \varepsilon_{n}^{\prime}$.

That the pair $\left(\mathcal{I}_{s}, \mathcal{I}_{t}\right)$ is disjoint is straightforward by properties (5) of Definition 38 and (L3) of Definition 44. Finally, $v_{2}^{2}\left(H_{t}, \mathcal{J}_{t}\right)>\theta$ implies that $v_{2}^{2}\left(H_{t}, \mathcal{I}_{s}^{(t)}\right)>$ $\theta-\varepsilon^{2}$, for all $t \in 2^{<\mathbb{N}}$. Hence by Lemma 11, we obtain that

$$
v_{2}^{2}\left(G_{s}, \mathcal{I}_{s}\right) \geq \sum_{t \in L_{s}}\left|L_{s}\right|^{-1} v_{2}^{2}\left(H_{t}, \mathcal{I}_{s}^{(t)}\right)-2^{-n} 2 M \varepsilon \geq \theta-(\varepsilon+2 M) \varepsilon=\theta^{\prime} .
$$

\subsection{The embedding of $S^{2}$ into $X$ when $\mathcal{M}_{X^{* *}}$ is non-separable.}

Lemma 46. Let $\left\{\mu_{\xi}\right\}_{\xi<\omega_{1}}$ be a non-separable subset of $\mathcal{M}^{+}[0,1]$. Then there is an uncountable subset $\Gamma$ of $\omega_{1}$ such that for every $\xi \in \Gamma, \mu_{\xi}=\lambda_{\xi}+\tau_{\xi}$, where $\lambda_{\xi}, \tau_{\xi}$ are positive Borel measures on $[0,1]$ satisfying the following properties.

(1) For all $\xi \in \Gamma, \lambda_{\xi} \perp \tau_{\xi}$ and $\left\|\tau_{\xi}\right\|>0$.

(2) For all $\zeta<\xi$ in $\Gamma, \mu_{\zeta} \perp \tau_{\xi}$.

Proof. We may suppose that for some $\delta>0,\left\|\mu_{\xi}-\mu_{\zeta}\right\|>\delta$, for all $0 \leq \zeta<\xi<\omega_{1}$. By transfinite induction we construct a strictly increasing sequence $\left(\xi_{\alpha}\right)_{\alpha<\omega_{1}}$ in $\omega_{1}$ such that for each $\alpha<\omega_{1}, \mu_{\xi_{\alpha}}=\lambda_{\xi_{\alpha}}+\tau_{\xi_{\alpha}}$, with $\lambda_{\xi_{\alpha}} \perp \tau_{\xi_{\alpha}},\left\|\tau_{\xi_{\alpha}}\right\|>0$ and $\tau_{\xi_{\alpha}} \perp \mu_{\xi_{\beta}}$ for all $\beta<\alpha$. The general inductive step of the construction is as follows. Suppose that for some $\alpha<\omega_{1},\left(\xi_{\beta}\right)_{\beta<\alpha}$ has been defined. Let $\left(\beta_{n}\right)_{n}$ be an enumeration of $\alpha$ and set

$$
\zeta_{\alpha}=\sup _{n} \xi_{\beta_{n}}, \quad \nu_{\alpha}=\sum_{n} \mu_{\xi_{\beta_{n}}} / 2^{n} \text { and } N_{\alpha}=\left\{\xi<\omega_{1}: \zeta_{\alpha}<\xi \text { and } \mu_{\xi} \ll \nu_{\alpha}\right\} \text {. }
$$

By the Radon-Nikodym theorem, $\left\{\mu_{\xi}\right\}_{\xi \in N_{\alpha}}$ is isometrically contained in $L_{1}\left([0,1], \nu_{\alpha}\right)$ and therefore it is norm separable. Since we have assumed that $\left\|\mu_{\xi}-\mu_{\zeta}\right\|>\delta$, for all $0 \leq \zeta<\xi<\omega_{1}$, we get that $N_{\alpha}$ is countable. Hence we can choose $\xi_{\alpha}>\sup N_{\alpha}$. Let $\mu_{\xi_{\alpha}}=\lambda_{\xi_{\alpha}}+\tau_{\xi_{\alpha}}$ be the Lebesgue analysis of $\mu_{\xi_{\alpha}}$, where $\lambda_{\xi_{\alpha}} \ll \nu_{\alpha}$ and $\tau_{\xi_{\alpha}} \perp \nu_{\alpha}$. By the definition of $\nu_{\alpha}$ and $\xi_{\alpha}$, we have that $\left\|\tau_{\xi_{\alpha}}\right\|>0, \tau_{\xi_{\alpha}} \perp \mu_{\xi_{\beta}}$, for all $\beta<\alpha$ and the inductive step of the construction has been completed.

Lemma 47. Let $\left\{\tau_{\xi}\right\}_{\xi<\omega_{1}}$ be an uncountable family of pairwise singular positive Borel measures on $[0,1]$. Then for every finite family $\left(\Gamma_{i}\right)_{i=1}^{k}$ of pairwise disjoint uncountable subsets of $\omega_{1}$ and every $\varepsilon>0$, there exist a family $\left(\Gamma_{i}^{\prime}\right)_{i=1}^{k}$ with $\Gamma_{i}^{\prime}$ an uncountable subset of $\Gamma_{i}$ and a family $\left(U_{i}\right)_{i=1}^{k}$ of open and pairwise disjoint subsets of $[0,1]$ such that $\tau_{\xi}\left([0,1] \backslash U_{i}\right)<\varepsilon$, for all $1 \leq i \leq k$ and $\xi \in \Gamma_{i}^{\prime}$.

Proof. For every $\alpha<\omega_{1}$, we choose $\left(\xi_{i}^{\alpha}\right)_{i=1}^{k} \in \prod_{i=1}^{k} \Gamma_{i}$ such that for every $\alpha \neq \beta$ in $\omega_{1}$ and every $1 \leq i \leq k, \xi_{i}^{\alpha} \neq \xi_{i}^{\beta}$. For each $0 \leq \alpha<\omega_{1}$ the $k$-tuple $\left(\tau_{\xi_{i}^{\alpha}}\right)_{i=1}^{k}$ consists of pairwise singular measures and so we may choose a $k$-tuple $\left(U_{i}^{\alpha}\right)_{i=1}^{k}$ of open subsets of $[0,1]$ with the following properties: (a) for each $i, \tau_{\xi_{i}^{\alpha}}\left([0,1] \backslash U_{i}^{\alpha}\right)<\varepsilon$; (b) for all $i \neq j, U_{i}^{\alpha} \cap U_{j}^{\alpha}=\emptyset$; (c) for each $i, U_{i}^{\alpha}$ is a finite union of open in $[0,1]$ intervals with rational endpoints.

Since the family of all finite unions of open intervals with rational endpoints is countable, there is a $k$-tuple $\left(U_{i}\right)_{i}$ and an uncountable subset $\Gamma$ of $\omega_{1}$, such that for all $1 \leq i \leq k$ and all $\alpha \in \Gamma, U_{i}^{\alpha}=U_{i}$. For each $1 \leq i \leq k$, set $\Gamma_{i}^{\prime}=\left\{\xi_{i}^{\alpha}: \alpha \in \Gamma\right\}$. Then for each $1 \leq i \leq k$ and all $\xi \in \Gamma_{i}^{\prime}, \tau_{\xi}\left([0,1] \backslash U_{i}\right)<\varepsilon$. 
Lemma 48. Let $X$ be a subspace of $V_{2}^{0}$ and suppose that $X^{* *}$ contains an uncountable family $\mathcal{F}$ such that $D_{\mathcal{F}}=\bigcup_{f \in \mathcal{F}} D_{f}$ is countable and $\mathcal{M}_{\mathcal{F}}=\left\{\mu_{f}\right\}_{f \in \mathcal{F}}$ is non-separable. Then there are constants $(M, \Lambda, \theta)$ such that for every $\varepsilon>0$ and every sequence $\left(\varepsilon_{n}\right)_{n}$ of positive scalars there is an $\left(\varepsilon,\left(\varepsilon_{n}\right)_{n}\right)-S^{2}$-generating system $\left(\left(H_{s}, \mu_{s}, \mathcal{J}_{s}\right)_{s \in 2^{<N}},\left(\mathcal{P}_{n}\right)_{n}\right)$ with constants $(M, \Lambda, \theta)$ and $H_{s} \in X$, for all $s \in 2^{<\mathbb{N}}$.

Proof. Since for all $f \in V_{2}$ and $\lambda \in \mathbb{R}, \mu_{\lambda f}=\lambda^{2} \mu_{f}$, we may assume that $\mathcal{F} \subseteq S_{X^{* * *}}$. By Lemma 46, there is a non-separable subset $\left\{\mu_{\xi}\right\}_{\xi<\omega_{1}}$ of $\mathcal{M}_{\mathcal{F}}$ such that for all $0 \leq \xi<\omega_{1}, \mu_{\xi}=\lambda_{\xi}+\tau_{\xi}, \lambda_{\xi} \perp \tau_{\xi}$ and for all $\zeta<\xi, \mu_{\zeta} \perp \tau_{\xi}$. By passing to a further uncountable subset, we may also assume that there is $\theta_{0}>0$ such that $\left\|\tau_{\xi}\right\|>\theta_{0}$. We fix $\varepsilon>0$ and a sequence $\left(\varepsilon_{n}\right)_{n}$ of positive real numbers. We will construct the following objects:

(1) a Cantor scheme $\left(\Gamma_{s}\right)_{s}$ of uncountable subsets of $\omega_{1}$ (that is, for all $s \in 2^{<\mathbb{N}}$, $\Gamma_{s \frown 0} \cup \Gamma_{s^{\wedge} 1} \subseteq \Gamma_{s}$ and $\left.\Gamma_{s \frown 0} \cap \Gamma_{s^{\wedge} 1}=\emptyset\right)$,

(2) a family $\left(\left(\xi_{s}^{0}, \xi_{s}^{1}\right)\right)_{s}$ of pairs with $\xi_{s}^{0}<\xi_{s}^{1}$ in $\Gamma_{s}$, for all $s \in 2^{<\mathbb{N}}$,

(3) a Cantor scheme of open subsets $\left(V_{s}\right)_{s}$ of $[0,1]$,

(4) a family of functions $\left(H_{s}\right)_{s}$ in $X$,

(5) an increasing sequence $\left(\mathcal{P}_{n}\right)_{n}$ of finite subsets of $[0,1] \backslash D_{\mathcal{F}}$, and

(6) a family $\left(\mathcal{J}_{s}\right)_{s}$ in $\mathcal{A}$,

such that the following are satisfied.

(i) For every $\xi \in \Gamma_{s}, \tau_{\xi}\left(V_{s}\right)>\theta_{0} / 2$ and $\tau_{\xi}\left([0,1] \backslash V_{s}\right)<\left(\sum_{i=0}^{|s|} 2^{-(i+2)}\right) \theta_{0}$.

(ii) The measures $\mu_{\xi_{s}^{0}}$ and $\mu_{\xi_{s}^{1}}$ are $\mathrm{w}^{*}$-condensation points of $\left\{\mu_{\xi}\right\}_{\xi \in \Gamma_{s}}$.

(iii) For every $n \geq 1, s \in 2^{n}, \xi \in \Gamma_{s}$ and $\mathcal{I} \in \mathcal{F}\left(\mathcal{P}_{n-1}\right)$,

$$
\left|\left(\mu_{\xi}-\mu_{\xi_{s^{-}}(n)}\right)(\bigcup \mathcal{I})\right|<\frac{\varepsilon_{n-1}}{16}, \text { where } s^{-}=(s(1), \ldots, s(n-1)) .
$$

(iv) $\left\|H_{s}\right\|_{V_{2}} \leq 2, \bigcup \mathcal{J}_{s} \subseteq V_{s}$ and $v_{2}^{2}\left(H_{s}, \mathcal{J}_{s}\right)>\theta_{0} / 2$

(v) For every $s \in 2^{n},\left\|H_{s}\right\|_{\infty} \leq \varepsilon_{n}$ and $v_{2}^{2}\left(H_{s}, \mathcal{I}\right) \leq 8\left(\mu_{\xi_{s}^{0}}+\mu_{\xi_{s}^{1}}\right)(\bigcup \mathcal{I})+\varepsilon_{n}$, for all $\mathcal{I} \in \mathcal{F}\left(\mathcal{P}_{n}\right)$.

(vi) If $\left(s_{i}\right)_{i=1}^{2^{n}}$ is the lexicographical enumeration of $\{0,1\}^{n}$, then $\left(H_{s_{i}}\right)_{i=1}^{2^{n}}$ is $\left(\varepsilon / 2^{i}\right)_{i=1}^{2^{n}}$-biorthogonal.

(vii) The set $\mathcal{P}_{n} \varepsilon_{n}$-determines the quadratic variation of $\left\langle\left\{H_{s}\right\}_{s \in 2^{<N}}\right\rangle$.

Given the above construction, we set $\mu_{s}=8\left(\mu_{\xi_{s}^{0}}+\mu_{\xi_{s}^{1}}\right)$ and we claim that the family $\left(\left(H_{s}, \mu_{s}, \mathcal{J}_{s}\right)_{s \in 2<\mathbb{N}},\left(\mathcal{P}_{n}\right)_{n}\right)$ is an $\left(\varepsilon,\left(\varepsilon_{n}\right)_{n}\right)-S^{2}$-generating system with constants $(M, \Lambda, \theta)$, where $M=2, \Lambda=16$ and $\theta=\theta_{0} / 2$. We only verify condition (41) of Definition 43 . since the other conditions are immediate. So let $n<m, s \in 2^{n}$ and $s_{0}, s_{1} \in 2^{m}$, with $s^{\urcorner} 0 \sqsubseteq s_{0}$ and $s^{\wedge} 1 \sqsubseteq s_{1}$. Then $\Gamma_{s_{0}} \subseteq \Gamma_{s^{\wedge} 0}, \Gamma_{s_{1}} \subseteq \Gamma_{s^{\wedge} 1}$, and so by (iii), for all $\mathcal{I} \in \mathcal{F}\left(\mathcal{P}_{n}\right)$ and $j \in\{0,1\}$, we get that

$$
\max \left\{\left|\left(\mu_{\xi_{s_{0}}^{j}}-\mu_{\xi_{s}^{0}}\right)(\bigcup \mathcal{I})\right|,\left|\left(\mu_{\xi_{s_{1}}^{j}}-\mu_{\xi_{s}^{1}}\right)(\bigcup \mathcal{I})\right|\right\} \leq \frac{\varepsilon_{n}}{16}
$$

Since

$$
\left|\frac{\mu_{s_{0}}+\mu_{s_{1}}}{2}-\mu_{s}\right| \leq 4\left(\left|\mu_{\xi_{s_{0}}^{0}}-\mu_{\xi_{s}^{0}}\right|+\left|\mu_{\xi_{s_{0}}^{1}}-\mu_{\xi_{s}^{0}}\right|+\left|\mu_{\xi_{s_{1}}^{0}}-\mu_{\xi_{s}^{1}}\right|+\left|\mu_{\xi_{s_{1}}^{1}}-\mu_{\xi_{s}^{1}}\right|\right),
$$

by (42), we have that for all $\mathcal{I} \in \mathcal{F}\left(\mathcal{P}_{n}\right),\left|\left(\frac{\mu_{s_{0}}+\mu_{s_{1}}}{2}-\mu_{s}\right)(\bigcup \mathcal{I})\right| \leq \varepsilon_{n}$. 
We now present the general inductive step of the construction. Let us suppose that the construction has been carried out for all $s \in 2^{<n}$. For every $s=$ $(s(1), \ldots, s(n)) \in 2^{n}$, we define

$$
\Gamma_{s}^{(1)}=\left\{\xi \in \Gamma_{s^{-}}: \forall \mathcal{I} \in \mathcal{F}\left(\mathcal{P}_{n-1}\right),\left|\left(\mu_{\xi}-\mu_{\xi_{s^{-}}^{s(n)}}\right)(\bigcup \mathcal{I})\right|<\varepsilon_{n} / 16\right\} .
$$

Since $\mathcal{F}\left(\mathcal{P}_{n-1}\right)$ is a finite subset of $\mathcal{F}\left([0,1] \backslash D_{X^{* *}}\right)$ and $\mu_{\xi}(\partial(\cup \mathcal{I}))=0$, for all $\mathcal{I} \in \mathcal{F}\left([0,1] \backslash D_{X^{* *}}\right.$ ) and all $\xi<\omega_{1}$ (where $\partial(\bigcup \mathcal{I})$ is the boundary of $\bigcup \mathcal{I}$ ), the set $\left\{\mu_{\xi}: \xi \in \Gamma_{s}^{(1)}\right\}$ is a relatively $\mathrm{w}^{*}$-open neighborhood of $\mu_{\xi_{s^{-}}(n)}$ in $\left\{\mu_{\xi}\right\}_{\xi \in \Gamma_{s^{-}}}$. By our inductive assumption, $\mu_{\xi_{s^{-}}^{0}}$ and $\mu_{\xi_{s}^{1}}$ are $\mathrm{w}^{*}$-condensation points of $\left\{\mu_{\xi}\right\}_{\xi \in \Gamma_{s^{-}}}$ and therefore for all $s \in 2^{n}$ the set $\Gamma_{s}^{(1)}$ is uncountable. Applying Lemma 47 we obtain a $2^{n}$-tuple $\left(U_{s}\right)_{s \in 2^{n}}$ of pairwise disjoint open subsets of $[0,1]$ and a family $\left(\Gamma_{s}^{(2)}\right)_{s \in 2^{n}}$ such that for each $s \in 2^{n}, \Gamma_{s}^{(2)}$ is an uncountable subset of $\Gamma_{s}^{(1)}$ and for all $\xi \in \Gamma_{s}^{(2)}$,

$$
\tau_{\xi}\left([0,1] \backslash U_{s}\right)<\theta_{0} / 2^{n+2}
$$

For every $s \in 2^{n}$ we set $V_{s}=U_{s} \cap V_{s^{-}}$. Since $\Gamma_{s}^{(2)} \subseteq \Gamma_{s}^{(1)} \subseteq \Gamma_{s^{-}}$, using (i), we get that for all $s \in 2^{n}$ and all $\xi \in \Gamma_{s}^{(2)}$,

$$
\tau_{\xi}\left([0,1] \backslash V_{s}\right)<\left(\sum_{i=0}^{n} 2^{-(i+2)}\right) \theta_{0} .
$$

Moreover as $\left(\sum_{i=0}^{n} 2^{-(i+2)}\right) \theta_{0}<\theta_{0} / 2$ and $\left\|\tau_{\xi}\right\|>\theta_{0}$, we get that for all $\xi \in \Gamma_{s}^{(2)}$,

$$
\tau_{\xi}\left(V_{s}\right)>\theta_{0} / 2 \text {. }
$$

Since for all $\zeta<\xi<\omega_{1}$, we have $\mu_{\xi} \geq \tau_{\xi}$ and $\tau_{\xi} \perp \mu_{\zeta}$, by Lemma 17] we get that $\mu_{f_{\xi}-f_{\zeta}} \geq \tau_{\xi}$. Therefore

$$
\mu_{f_{\xi}-f_{\zeta}}\left(V_{t}\right)>\tau_{\xi}\left(V_{t}\right)>\theta_{0} / 2
$$

for all $s \in 2^{n}$ and $\zeta<\xi$ in $\Gamma_{s}^{(2)}$.

Let $\left(s_{i}\right)_{i=1}^{2^{n}}$ be the lexicographical enumeration of $2^{n}$. Using Lemma 8 , Proposition 4 and a finite induction on $1 \leq i \leq 2^{n}$, we will choose for every $1 \leq i \leq 2^{n}$, the set $\Gamma_{s_{i}}$, the function $H_{s_{i}}$, the pair of ordinals $\left(\xi_{s_{i}}^{0}, \xi_{s_{i}}^{1}\right)$ and the family $\overline{\mathcal{J}}_{s_{i}}$ satisfying (ii)-(vi). Suppose that for some $1 \leq k<2^{n},\left(H_{s_{i}}\right)_{i \leq k}$ have been chosen so that $\left(H_{s_{i}}\right)_{i \leq k}$ is an $\left(\left(\varepsilon_{i}^{k}\right),\left(\delta_{i}\right)_{i=0}^{k-1}\right)$-biorthogonal sequence, where $\varepsilon_{i}^{k}=\left(\sum_{r=1}^{k-1+1} 2^{-r}\right) \varepsilon / 2^{i}$. Then by Lemma 8 there are $\delta_{m}>0$ and $\epsilon>0$ such that for every $H \in V_{2}^{0}$ with $\|H\|_{\infty}<\epsilon$, the sequence $H_{s_{1}}, \ldots, H_{s_{m-1}}, H$ is an $\left(\left(\varepsilon_{i}^{k+1}\right)_{i=1}^{k+1},\left(\delta_{i}\right)_{i=0}^{m}\right)$-biorthogonal sequence. Clearly, we may suppose that $\epsilon<\varepsilon_{n}$. For each $\xi<\omega_{1}$, let $f_{\xi} \in \mathcal{F}$ such that $\mu_{\xi}=\mu_{f_{\xi}}$. Since $D_{\mathcal{F}}$ is countable, by Proposition 4 , we have that $\left(\mathcal{F},\|\cdot\|_{\infty}\right)$ is separable and so there is an uncountable subset $\Gamma_{t_{k+1}}^{(3)}$ of $\Gamma_{t_{k+1}}^{(2)}$ such that for all $\zeta, \xi$ in $\Gamma_{t_{k+1}}^{(3)}$,

$$
\left\|f_{\xi}-f_{\zeta}\right\|_{\infty}<\epsilon / 8 \text {. }
$$

Also applying Lemma 36] for the family $\mathcal{F}=\left\{f_{\xi}\right\}_{\xi \in \Gamma_{s_{k+1}}^{(3)}}$ we pass to a further uncountable subset $\Gamma_{s_{k+1}}^{(4)}$ of $\Gamma_{s_{k+1}}^{(3)}$ such that for every $\zeta, \xi \in \Gamma_{s_{k+1}}^{(4)}$,

$$
\left\|\mu_{f_{\xi}-f_{\zeta}}^{d}\right\|<(\epsilon / 128)^{2} \text {. }
$$


We set $\Gamma_{s_{k+1}}=\Gamma_{s_{k+1}}^{(4)}$ and we choose $\xi_{s_{k+1}}^{0}<\xi_{s_{k+1}}^{1}$ in $\Gamma_{s_{k+1}}$ such that $\mu_{\xi_{s_{k+1}}^{0}}$ and $\mu_{\xi_{s_{k+1}}^{1}}$ are $\mathrm{w}^{*}$-condensation points of the set $\left\{\mu_{\xi}\right\}_{\xi \in \Gamma_{s_{k+1}}}$. We put $F=f_{\xi_{s_{k+1}}^{0}}-$ $f_{\xi_{s_{k+1}}^{1}}$. Since for all $\xi<\omega_{1},\left\|f_{\xi}\right\|_{V_{2}}=1$, we have that $\|F\|_{\infty} \leq 2$. Moreover by (47)-(49), we have that

$$
\mu_{F}\left(V_{s_{k+1}}\right)>\theta_{0} / 2,\|F\|_{\infty}<\epsilon / 12 \text {, and }\left\|\mu_{F}^{d}\right\|<(\epsilon / 128)^{2} .
$$

Let $\left(f_{n}\right)_{n}$ be a sequence in $X$ pointwise converging to $F$ with $\left\|f_{n}\right\|_{V_{2}} \leq\|F\|_{V_{2}}$ (see Remark 7). By Proposition 29, there exist a convex block sequence $\left(h_{n}\right)_{n}$ of $\left(f_{n}\right)_{n}$ and $\mathcal{J} \in \mathcal{A}$ such that setting $H=h_{l}-h_{k}$, for sufficiently large $k<l$, we have that

(a) $\|H\|_{V_{2}} \leq 2\|F\|_{V_{2}} \leq 4$ and $\|H\|_{\infty} \leq 4\|F\|_{\infty}+\epsilon / 2 \leq \epsilon$,

(b) $\bigcup \mathcal{J} \subseteq V_{s_{k+1}}$ and $v_{2}^{2}(H, \mathcal{J})>\theta_{0} / 2$,

(c) for all $\mathcal{I} \in \mathcal{A}$,

$$
v_{2}^{2}(H, \mathcal{I}) \leq 4 \mu_{F}(\bigcup \mathcal{I})+32\|f\|_{V_{2}} \sqrt{\left\|\mu_{F}^{d}\right\|}+\epsilon / 2 \leq 8\left(\mu_{\xi_{s_{k+1}^{0}}^{0}}+\mu_{\xi_{s_{k+1}}^{1}}\right)(\bigcup \mathcal{I})+\epsilon .
$$

We set $H_{s_{k+1}}=H$ and $J_{s_{k+1}}=J$ and the inductive step is completed. Finally, using Proposition 1, we choose a sufficiently dense finite subset $\mathcal{P}_{n} \subseteq[0,1] \backslash D_{\mathcal{F}}$ determining the quadratic variation of $\left\langle\left\{H_{s}\right\}_{s \in 2^{n}}\right\rangle$ which completes the proof of the inductive step.

Lemma 48 Proposition 45 and Proposition 42 yield the following.

Proposition 49. Let $X$ be a subspace of $V_{2}^{0}$ and suppose that $X^{* *}$ contains an uncountable family $\mathcal{F}$ such that $D_{\mathcal{F}}=\bigcup_{f \in \mathcal{F}} D_{f}$ is countable and $\mathcal{M}_{\mathcal{F}}=\left\{\mu_{f}\right\}_{f \in \mathcal{F}}$ is non-separable. Then $X$ contains a subspace isomorphic to the space $S^{2}$.

In the next lemma and proposition we state some results concerning the space $T F$ and the tree families generating this space. We refer the reader to [1] for the relevant notation.

Lemma 50. Let $\mathcal{G}=\left(\left(g_{s}\right),\left(I_{s}, J_{s}\right)\right)_{s \in 2<\mathbb{N}}$ be a tree family such that $T F=\overline{\left\langle\left\{g_{s}\right\}_{s}\right\rangle}$ and for every $n \geq 0$, let $K_{n}=\bigcup_{s \in 2^{n}} I_{s}$. Then for every $f \in T F^{* *}$, supp $\mu_{f} \subseteq K$, where $K=\bigcap_{n=0}^{\infty} K_{n}$.

Proof. Let $f \in T F^{* *}$ and let $\left(f_{m}\right)_{m}$ be a sequence in $\left\langle\left\{g_{s}\right\}_{s}\right\rangle$ pointwise convergent to $f$ and such that $\left\|f_{n}\right\|_{V_{2}} \leq\|f\|_{V_{2}}$. For each $n \geq 0$, let $P_{n}: T F^{* *} \rightarrow G_{n}$ be the natural projection onto the finite-dimensional space $G_{n}=\left\langle\left\{g_{s}\right\}_{|s|<n}\right\rangle$ (where $\left.G_{0}=\{0\}\right)$. Also let $h_{m}^{n}=f_{m}-P_{n}\left(f_{m}\right)$ and $h_{n}=f-P_{n}(f)$. Since $P_{n}$ is $\mathrm{w}^{*}$-w* continuous, the sequence $\left(h_{m}^{n}\right)_{m}$ is pointwise convergent to $h_{n}$ and so supp $h_{n} \subseteq K_{n}$. Since $\mu_{h_{n}}=\mu_{f}$ and supp $\mu_{h_{n}} \subseteq \operatorname{supp} h_{n}$, we conclude that supp $\mu_{f} \subseteq K_{n}$ for all $n \geq 0$.

Proposition 51. The set $\mathcal{M}_{T F^{* *}}^{c}=\left\{\mu_{f}: f \in T F^{* *} \cap C[0,1]\right\}$ is a non-separable subset of $\mathcal{M}[0,1]$. Therefore the space $S^{2}$ is embedded into $T F$.

Proof. Let $\mathcal{G}=\left(\left(g_{s}\right),\left(I_{s}, J_{s}\right)\right)_{s \in 2<\mathbb{N}}$ be a tree family such that $T F=\overline{\left\langle\left\{g_{s}\right\}_{s}\right\rangle}$. Also let $\left(T_{\sigma}\right)_{\sigma \in 2^{\mathbb{N}}}$ be the almost disjoint family of dyadic subtrees in $2^{<\mathbb{N}}$ defined in Remark 13. For each $\sigma \in 2^{\mathbb{N}}$, we set $\mathcal{G}_{\sigma}=\left(\left(g_{s}\right),\left(I_{s}, J_{s}\right)\right)_{s \in T_{\sigma}}$. Since $\mathcal{G}_{\sigma}$ is also a tree family, the space $X_{\sigma}=\overline{\left\langle\left\{g_{s}\right\}_{s \in T_{\sigma}}\right\rangle}$ is a copy of $T F$. Therefore $c_{0}$ is embedded into $X_{\sigma}$ which gives that $\left(X_{\sigma}^{* *} \backslash X\right) \cap C[0,1] \neq \emptyset$ (cf. Remark 9). So for each $\sigma \in 2^{\mathbb{N}}$, we can pick an $f_{\sigma} \in X_{\sigma}^{* *} \backslash X \cap C[0,1]$. Setting $T_{\sigma}=\left(t_{s}^{\sigma}\right)_{s \in 2^{\mathbb{N}}}$ and $K_{\sigma}=$ $\bigcap_{n} \bigcup_{s \in 2^{n}} I_{t_{s}^{\sigma}}$, by Lemma 50 we have that supp $\mu_{f_{\sigma}} \subseteq K_{\sigma}$. Since $\left(T_{\sigma}\right)_{\sigma \in 2^{\mathbb{N}}}$ is 
an almost disjoint family, we get that $\left(K_{\sigma}\right)_{\sigma \in 2^{\mathbb{N}}}$ is a disjoint family of compact subsets of $[0,1]$ and so $\left\{\mu_{f_{\sigma}}\right\}_{\sigma \in 2^{\mathbb{N}}}$ consists of pairwise singular positive measures. As $\left\{f_{\sigma}\right\}_{\sigma \in 2^{\mathbb{N}}} \subseteq T F^{* *} \cap C[0,1]$, we conclude that $\mathcal{M}_{T F^{* *}}^{c}$ is non-separable. Finally, that $S^{2}$ is embedded into $X$ follows by Proposition 49, for $\mathcal{F}=T F^{* *} \cap C[0,1]$.

Proposition 52. Let $X$ be a subspace of $V_{2}^{0}$ such that the space $S^{2}$ is embedded into $X$. Then the set $\mathcal{M}_{X^{* *}}=\left\{\mu_{f}: f \in X^{* *}\right\}$ is a non-separable subset of $\mathcal{M}[0,1]$.

Proof. Let $T$ be an isomorphic embedding of $S^{2}$ into $X$ and let $f_{s}=T\left(e_{s}\right)$, where $\left(e_{s}\right)_{s}$ is the usual basis of $S^{2}$. From [3] we have that for each $\sigma \in 2^{\mathbb{N}}$, the sequence $\left(\sum_{k=0}^{n} f_{\sigma \mid k}\right)_{n}$ is pointwise converging to a function $f_{\sigma} \in\left(X^{* *} \backslash X\right) \cap C[0,1]$. Hence there exist an uncountable subset $\Sigma \subseteq 2^{\mathbb{N}}$ and $\delta>0$ such that for all $\sigma \in \Sigma$, $\operatorname{dist}\left(f_{\sigma}, X\right)>\delta$. We will show that the set $\left\{\mu_{f_{\sigma}}: \sigma \in \Sigma\right\} \subseteq \mathcal{M}[0,1]$ is nonseparable. Indeed, otherwise, we can choose a norm-condensation point $\mu \in \mathcal{M}[0,1]$ of $\left\{\mu_{\sigma}: \sigma \in 2^{\mathbb{N}}\right\}$. Also fix a positive integer $m \in \mathbb{N}$ and $\varepsilon>0$. Then for uncountably many $\sigma \in \Sigma$, we have that

$$
\left\|\mu_{f_{\sigma}}-\mu\right\| \leq \varepsilon / m
$$

Let $\sigma_{1}, \ldots, \sigma_{m} \in \Sigma$ satisfy (51) and let $n_{0} \in \mathbb{N}$ be such that for all $n \geq n_{0}$ and $1 \leq i<j \leq m, \sigma_{i}\left|n \perp \sigma_{j}\right| n$. We set $g_{\sigma_{i}}=\sum_{n>n_{0}} f_{\sigma_{i} \mid n}$. Since $f_{\sigma_{i}}-g_{\sigma_{i}}=$ $\sum_{n<n_{0}} f_{\sigma_{i} \mid n} \in X$, we have that $\operatorname{dist}\left(g_{\sigma_{i}}, X\right)=\operatorname{dist}\left(f_{\sigma_{i}}, X\right)>\delta$ and $\mu_{f_{\sigma_{i}}}=\mu_{g_{\sigma_{i}}}$. For every $n \in \mathbb{N}$, let $F_{n}^{i}=\sum_{k=n_{0}}^{n_{0}+n} f_{\sigma_{i} \mid k}$. Then

$$
\left\|F_{n}^{i}\right\|_{V_{2}} \leq\|T\|\left\|\sum_{k=n_{0}}^{n_{0}+n} e_{\sigma_{i} \mid k}\right\|_{S^{2}} \leq\|T\| .
$$

Applying Proposition 34 for the continuous function $g_{\sigma_{i}} \in X^{* *} \backslash X$, the sequence $\left(F_{n}^{i}\right)_{n}$ and $\varepsilon_{n}=\varepsilon / m 2^{n}$, we obtain a convex block sequence $\left(h_{n}^{i}\right)_{n}$ of $\left(F_{n}^{i}\right)_{n}$ such that the functions $G_{n}^{i}=h_{2 n+1}^{i}-h_{2 n}^{i}$ satisfy the following. (i) $\delta<\left\|G_{n}^{i}\right\|_{V_{2}} \leq 2\|T\|$, (ii) $\left\|G_{n}^{i}\right\|_{\infty}<\varepsilon /\left(m 2^{2 n}\right)$ and (iii) for every $\mathcal{I} \in \mathcal{A}, v_{2}^{2}\left(G_{n}^{i}, \mathcal{I}\right) \leq 4 \mu_{f_{\sigma_{i}}}(\bigcup \mathcal{I})+\varepsilon /\left(m 2^{2 n}\right)$.

By (ii) and Lemma 8 , we can choose $n_{1}<\ldots<n_{m}$ such that the finite sequence $\left(G_{n_{i}}^{i}\right)_{i=1}^{m}$ is $\varepsilon / m$-biorthogonal. By the definition of $G_{n}^{i}$, we have that $G_{n_{i}}^{i}=\sum_{s \in F_{i}} \lambda_{s} f_{s}$, where $F_{i}$ is a finite subset of $\left\{\sigma_{i} \mid n: n \in \mathbb{N}\right\}$. Hence

$$
\delta<\left\|G_{n_{i}}^{i}\right\|_{V_{2}} \leq\|T\|\left\|\sum_{s \in F_{i}} \lambda_{s} e_{s}\right\|_{S^{2}} \leq\|T\| \max _{s \in F_{i}}\left|\lambda_{s}\right| .
$$

Let $s_{i} \in F_{i}$ be such that $\left|\lambda_{s_{i}}\right|=\max _{s \in F_{i}}\left|\lambda_{s}\right|$. Then by (52), $\left|\lambda_{s_{i}}\right| \geq \delta /\|T\|$ and so, since the set $\left\{s_{i}: 1 \leq i \leq m\right\}$ is an antichain of $2^{<\mathbb{N}}$, we get that

$$
\left\|\sum_{i=1}^{m} G_{n_{i}}^{i}\right\|_{V_{2}} \geq \frac{1}{\left\|T^{-1}\right\|}\left\|\sum_{i=1}^{m} \sum_{s \in F_{i}} \lambda_{s} e_{s}\right\|_{S^{2}} \geq \frac{1}{\left\|T^{-1}\right\|} \sqrt{\frac{m \delta^{2}}{\|T\|^{2}}} \geq \frac{\delta \sqrt{m}}{\left\|T^{-1}\right\|\|T\|} .
$$

By Lemma 11 and (iii), for every $\mathcal{I} \in \mathcal{A}$ we have that

$$
\begin{aligned}
v_{2}^{2}\left(\sum_{i=1}^{m} G_{n_{i}}^{i}, \mathcal{I}\right) & \leq \sum_{i=1}^{m} v_{2}^{2}\left(G_{n_{i}}^{i}, \mathcal{I}^{(i)}\right)+(4\|T\|+1) \varepsilon \leq 4 \sum_{i=1}^{m} \mu_{f_{\sigma_{i}}}\left(\bigcup \mathcal{I}^{(i)}\right)+(4\|T\|+2) \varepsilon \\
& \leq 4 \mu(\bigcup \mathcal{I})+\varepsilon+(4\|T\|+2) \varepsilon . \leq 4\|\mu\|+(4\|T\|+3) \varepsilon .
\end{aligned}
$$

Therefore, letting $\varepsilon \rightarrow 0,4\|\mu\| \geq\left\|\sum_{i=1}^{m} G_{n_{i}}^{i}\right\|_{V_{2}}$ and so by (153), we get a contradiction. 
We are finally ready to prove the main results of the paper.

Theorem 53. Let $X$ be a subspace of $V_{2}^{0}$. Then the space $S^{2}$ is embedded into $X$ if and only if $\mathcal{M}_{X^{* *}}$ is non-separable.

Proof. By Proposition 52 if $S^{2}$ is embedded into $X$, then $\mathcal{M}_{X^{* *}}$ is non-separable. Conversely suppose that $\mathcal{M}_{X^{* *}}$ is non-separable. Then we distinguish two cases. If $X^{*}$ is separable, then by Proposition 2, the set $D_{X^{* *}}$ is countable and hence by Proposition 49, for $\mathcal{F}=X^{* *}$, we get that $S^{2}$ is embedded into $X$. In the case that $X^{*}$ is non-separable, by [1, the space $T F$ is embedded into $X$. By Proposition 51 , we have that $S_{2}$ is embedded into $T F$ and hence into $X$.

Theorem 54. Let $X$ be a subspace of $V_{2}^{0}$. Then $c_{0}$ is embedded into $X$ if and only if $X^{* *}$ is non-separable.

Proof. Suppose that $X^{* *}$ is non-separable (the other direction is obvious). If $X^{*}$ is non-separable, then as we have already mentioned the space $T F$ and hence $c_{0}$ is embedded into $X$. So assume that $X^{*}$ is separable. We distinguish the following cases. If $\mathcal{M}_{X^{* *}}$ is non-separable, then the result follows by Theorem 53 . Otherwise, $\mathcal{M}_{X^{* *}}$ is separable and so by Proposition [37, $c_{0}$ is again embedded into $X$.

\section{REFERENCES}

[1] D. Apatsidis, S.A. Argyros, and V. Kanellopoulos, On the subspaces of JF and JT with non-separable dual, J. Funct. Anal., 254, 632-674, 2008. MR2381158

[2] S.A. Argyros and V. Kanellopoulos, Optimal sequences of continuous functions converging to a Baire-1 function, Math. Ann. 324, 689-729, 2002. MR.1942246 (2004d:46028)

[3] S.A. Argyros, A. Manoussakis and M. Petrakis, Function spaces not containing $\ell_{1}$, Israel J. Math., 135, 29-81, 2003. MR1996395 (2004f:46015)

[4] G. Bourdaud, M. Lanza de Cristoforis, and W. Sickel, Superposition operators and functions of bounded p-variation. II, Nonlinear Anal. 62, no. 3, 483-517, 2005. MR2147980 (2006g:47108)

[5] G. Bourdaud, M. Lanza de Cristoforis, and W. Sickel, Superposition operators and functions of bounded p-variation, Rev. Mat. Iberoam. 22, no. 2, 455-487, 2006. MR2294787 (2009c:46047)

[6] S. Buechler, James Function spaces, Ph.D. Thesis, University of Texas at Austin, 1994.

[7] N. Dew, Asymptotic structure in Banach spaces, Ph.D. Thesis, Oxford University, eprints.maths.ox.ac.uk/49/01/dew.pdf

[8] R.M. Dudley and R. Norvaisa, Differentiability of six operators on nonsmooth functions and p-variation, Lecture Notes in Mathematics, 1703, Springer-Verlag, Berlin, 1999. MR.1705318 (2000e:46053)

[9] B. I. Golubov, Continuous functions of bounded p-variation, Mat. Zametki 1, 305-312, 1967. MR0211181 (35:2063)

[10] R. Haydon, E. Odell and H.P. Rosenthal, On certain classes of Baire-1 functions with applications to Banach space theory, Lecture Notes in Math., vol.1470, Springer-Verlag, 1-35, 1991. MR:1126734 (92h:46018)

[11] R.C. James, A separable somewhat reflexive Banach space with non-separable dual, Bull. Amer. Math. Soc., 80, 738-743, 1974. MR0417763 (54:5811)

[12] A.S. Kechris, Classical Descriptive Set Theory, Graduate Texts in Mathematics, SpringerVerlag, 1995. MR1321597 (96e:03057)

[13] A.S. Kechris and A. Louveau, A classification of Baire class 1 functions, Trans. Amer. Math. Soc., 318, 209-236, 1990. MR946424 (90f:26005)

[14] S.V. Kisliakov, A remark on the space of functions of bounded p-variation, Math. Nachr., 119, 37-40, 1984. MR774183 (86m:46026)

[15] J. Lindenstrauss and C. Stegall, Examples of separable spaces which do not contain $\ell_{1}$ and whose duals are non-separable, Studia Math., 54, 81-105, 1975. MR0390720 (52:11543) 
[16] J. Lindenstrauss and L. Tzafriri, Classical Banach spaces I and II, Springer, 1996. MR0500056 (58:17766)

[17] E. R. Love, A generalization of absolute continuity, J. London Math. Soc. 26, 1-13, 1951. MR0039791 (12:599a)

[18] E. Odell and H.P. Rosenthal, A double dual characterization of separable Banach spaces containing $\ell_{1}$, Israel J. Math., 20, 375-387, 1975. MR0377482 (51:13654)

[19] F. Prus-Wisniowski, Continuity of p-variation in the Vietoris topology, J. Math. Anal. Appl. 340, no. 2, 1452-1468, 2008. MR2390943 (2009e:26016)

[20] C. A. Rogers, Hausdorff measures, Cambridge University Press, 1970. MR0281862 (43:7576)

[21] H. P. Rosenthal, A characterization of Banach spaces containing $c_{0}$, J. Amer. Math. Soc. 7, 707-748, 1994. MR1242455 (94i:46032)

[22] N. Wiener, The quadratic variation of a function and its Fourier coefficients, J. Math. and Phys. 3, 72-94, 1924.

[23] L. C. Young, An inequality of the Hölder type, connected with Stieltjes integration, Acta Math. 67 , no. 1, 251-282, 1936 MR 1555421

Department of Mathematics, Faculty of Applied Sciences, National Technical University of Athens, Zografou Campus, 157 80, Athens, Greece

E-mail address: dapatsidis@hotmail.com

Department of Mathematics, Faculty of Applied Sciences, National Technical University of Athens, Zografou Campus, 157 80, Athens, Greece

E-mail address: sargyros@math.ntua.gr

Department of Mathematics, Faculty of Applied Sciences, National Technical University of Athens, Zografou Campus, 157 80, Athens, Greece

E-mail address: bkanel@math.ntua.gr 